\title{
Unhappy working with men? Workplace gender diversity and job-related well-being in Britain 25
}

\author{
Getinet Astatike Haile * \\ University of Nottingham, Nottingham, UK \\ IZA, Bonn, Germany
}

\section{A R T I C L E I N F O}

\section{Article history:}

Received 17 March 2009

Received in revised form 15 November 2011

Accepted 6 February 2012

Available online 21 February 2012

\section{JEL classification:}

J16

J82

J7

I31

Keywords:

Gender diversity

Job-related well-being

Linked employer-employee data

Britain

\begin{abstract}
A B S T R A C T
This paper attempts to establish empirically the link between workplace gender diversity and employee jobrelated well-being in Britain. Using nationally representative linked employer-employee data and accounting for unobserved workplace heterogeneity the paper finds gender diversity to be associated with lower employee well-being for women. Workplace gender equality policies and practices are not found to ameliorate this finding.
\end{abstract}

(C) 2012 Elsevier B.V. Open access under CC BY license.

\section{Introduction}

The labour market participation of women has increased significantly in the industrialised world in recent years. In Britain, women's participation stood at $37.1 \%$ in 1971 but this has increased to $45.8 \%$ in 2005 (ONS, 2006). Inevitably, this has led to an increase in workplace gender diversity (WGD henceforth). The increase in WGD has been attributed to some important developments including demographic changes, tight labour market conditions, and regulatory measures. ${ }^{1}$

Despite the considerable change in WGD and the growing prominence of equality and diversity related discourses, there is a dearth of empirical literature on the links between WGD and employee job-related well-being (EJW henceforth). Intriguingly, the increasing diversity and interventions aimed at promoting diversity in Britain are taking place despite evidence of widespread gender discrimination (Booth, 2009; Arulampalam, et al., 2007; Berthoud and Blekesaune, 2007; Riach and Rich, 2006; Peccei and Lee, 2005; Jones et al., 2003; Pudney and Shields, 2000a, b; Wright and Ermisch, 1991). If gender discrimination is as

\footnotetext{
is The author would like to thank the editor and two anonymous referees for useful comments and suggestions on an earlier version of this paper.

* Division of Economics and Finance, Nottingham University Business School, North Building, Jubilee Campus, Wollaton Road, Nottingham, NG8 1BB, UK.

E-mail address: getinet.haile@nottingham.ac.uk.

1 Equality Act 2006, 2010.
}

widespread as the existing evidence suggests, the growing emphasis on diversity may have adverse implications on EJW, particularly if diversity is driven by labour cost considerations.

EJW is what employees feel about themselves in relation to their job. It is concerned with both physical and mental aspects of health. It is multidimensional in nature and forms an integral part of overall well-being (Warr, 1990, 1994, 1999; Rode, 2004; Wood, 2008). Job satisfaction is one aspect of EJW, which has been shown to be a powerful predictor of quits and absenteeism (Freeman, 1978; Akrelof et al., 1988; Clark et al., 1998; Clark, 2001; Levy-Garboua and Montmarquette, 2004; Levy-Garboua et al., 2007). The growing WGD in the face of widespread workplace gender discrimination may mean adverse EJW outcomes, with cost implications to employees, employers and society at large.

This paper attempts to establish empirically the link between WGD and EJW. It also explores whether workplace HRM policy and practices have any bearing on the gender-wellbeing link. Firmly establishing the link between WGD and EJW is crucial for two important reasons. First, the growing discourse on diversity is not matched by rigorous research. Secondly, there is conflicting evidence regarding the link between WGD and EJW.

The paper aims to fill gaps in the existing literature and has several strengths. First, it uses the WERS2004 data, the nationally representative linked employer-employee data, with large number of demographically varied workplaces located across Britain. The linked data also have extensive information on workplaces, employees, and 
HRM policy and practices. This enables controlling for observable influences on EJW much more comprehensively than has been done to date. Secondly, the data have extensive sets of measures on EJW that include eight facets of job satisfaction and six affective well-being measures. This allows investigating links between WGD and aspects of EJW hitherto unexplored. Third, WGD is measured as an index addressing nonlinearities existing studies in Britain do not account for. As recent U.S. based studies have demonstrated, using proportions of workers does not allow capturing nonlinear effects. ${ }^{2}$ Fourth, the paper exploits the nested structure of the WERS2004 data to control for unobserved workplace heterogeneity, something previous studies ignore. One important lesson research in labour economics, particularly where there is a matched employer-employee data, underscores is the importance of unobserved factors in determining labour market outcomes (see, for example, Abowd et al., 1999). In the workplace setting considered here, there may well be unmeasured aspects of workplaces that influence EJW, which this study attempts to address. The remainder of the paper is organised as follows. Section 2, makes a brief review of relevant existing literature. In Section 3, a description of the data and variables used in the empirical analyses will be made. Section 4 sets out the framework for the empirical analysis carried out. Section 5 discusses the empirical results obtained before the final section concludes the paper.

\section{Review of existing literature}

The literature on WGD is fairly limited and quite recent. Moreover, it has an almost exclusive focus on the USA and relates to the relationship between diversity and such economic outcomes as firm performance (Kurtulus, 2008; Leonard and Levine, 2006), turnover (Leonard and Levine, 2006; Giuliano et al., 2006) and promotions (Blau and DeVaro, 2007; Giuliano et al., 2006). Kurtulus (2008) examines the effect of grouping workers into heterogeneous divisions on worker and division performance using panel data from a large US firm and finds some evidence that gender heterogeneity is associated with higher worker performance. Leonard and Levine (2006) study the effect of gender diversity on turnover among sales workers in retail branches of a large U.S. firm and find evidence linking WGD and higher quit rates among women. Using the same data from the large US retail firm, Giuliano et al. (2006) study gender differences between managers and workers and find some evidence on worker quit rates. None of the three studies focuses on the well-being effect of WGD directly. However, the wellestablished link between job satisfaction and quit behaviour (for e.g. Freeman, 1978; Akerlof et al., 1988; Clark, 2001) may mean that the latter two studies imply adverse relationship between EJW and WGD.

A number of other studies analyses the relationship between workgroup gender composition and employee job satisfaction. Fields and Blum (1997) look at the relationship between workgroup gender composition and job satisfaction using US data and find both men and women working in gender-balanced groups to have higher levels of job satisfaction vis-à-vis their counterparts in homogeneous groups. On the other hand, Peccei and Lee (2005), using WERS98, find gender homogeneity to be positively related to job satisfaction, particularly for men. Using the BHPS, Rose (2005) finds a sharp decline in female job satisfaction over the 1990s, a pattern that may be linked to the recent increase in the share of women in the labour market. ${ }^{3}$ Establishing

\footnotetext{
${ }^{2}$ A (fully) gender diverse workplace should have men and women with a 50:50 proportion. An increase in the proportions of women beyond $50 \%$ means the workplace becomes less diverse, although a workplace with higher proportions of women might be regarded as diverse on the supposition that such a workplace has given more space for the traditionally disadvantaged gender group.

3 Other (sociological) studies of similar nature, for example Wharton and Baron (1987), find men in mixed work settings to have lower job-related satisfaction and self-esteem and more job-related depression than men in either male- or femaledominated work settings and attribute this to the decline in quantity and quality of inter-group relations as groups become more balanced.
}

whether there is a link between WGD and EJW may be of considerable relevance for workplace well-being. ${ }^{4}$

There are several psychological and sociological studies that attempt to explain how differences between workgroup members along gender and other dimensions influence the attitude and subjective well-being of group members. However, most of these studies neither account for confounding influences nor use large and representative data (Knippenberg and Schippers, 2007; Maume and Sebastian, 2007; Jackson et al., 2003; Fields and Blum, 1997). Jackson et al. (2003) also report that in their review of 63 studies for the period 1997-2002 they found very little focus on affective outcomes. Peccei and Lee (2005) found eight US studies assessing the relation of gender proportions to satisfaction, but similarly noted the paucity of control information in these studies.

The review in the preceding paragraphs points to lack of consensus regarding the link between WGD and EJW. This could be due to several reasons including: the nature of the data used, whether confounding factors are accounted for sufficiently, the nature of the well-being measure(s) used, the way WGD is measured and the type of empirical methodology employed. On data, Leonard and Levine (2006) state that an ideal diversity study would (i) have a large number of demographically varied workplaces, (ii) control for location to capture differences in the local labour market condition, (iii) minimise confounding variations across workplaces in HRM policy and practice and workplace and job characteristics, and (iv) assign randomly different demographic mixes to each workplace.

This paper uses data that address nearly all of these data related concerns by controlling for a range of employee, workplace, HRM policy and practice, and geographic influences much more comprehensively than in any previous study. ${ }^{5}$ Extensive sets of job-relate wellbeing measures have also been used. Although there are few studies that dwell on single item measures of job satisfaction to study the effects of workgroup demographic composition, the use in this paper of eight different facets of job satisfaction provides the richness one would need to address possible sensitivities stemming from the way well-being is measured. This study also uses measures of affective well-being that have not been used in previous studies investigating WGD and EJW. That the paper employs alternative econometric models and accounts for unobserved heterogeneity is a further strength. With few exceptions (for example, Leonard and Levine, 2006; Kurtulus, 2008), most of the studies investigating the issue of workgroup composition do not account for unobserved heterogeneity. Also, in most of the existing literature WGD is measured as the proportion of women at the workplace. As detailed in the next section, such a measure may not fully capture the dynamics associated with WGD.

\section{Data and variables}

\subsection{Overview of the data}

The data used in this paper come from the 2004 British Workplace Employment Relations Survey (WERS2004), the most authoritative sources of information on employment relations in Great Britain. It offers linked employer-employee data representative of all workplaces with five or more employees (Kersley et al., 2006). The survey covers a whole host of issues relating to both employers and employees, allowing control on a battery of individual and workplace level attributes. The final estimation sample in this paper comprises of 18,064 employees in 1506 workplaces. This is from the initial matched sample of 22,451

\footnotetext{
${ }^{4}$ For example, Kochan et al. (2003) reported adverse effects of racial diversity on team processes being mitigated through training and development-focused initiatives.

${ }^{5}$ Controlling for as many observable workplace and employee influences as possible has been emphasised in other previous studies (Wood, 2008; Peccei and Lee, 2005). Maume and Sebastian (2007) find job satisfaction of whites being negatively related to the proportion of minority workers in the absence of controls, but the effect disappears when job characteristics are controlled for.
} 
employees in 1733 workplaces. The reduction in sample size is the result of: (i) missing values in any one of the reported EJW outcomes, (ii) missing values in any one of the employee and workplace covariates, including the WGD measure and (iii) retaining only workplaces with at least two responding employees.

\subsection{Definition of variables}

\subsubsection{Outcome variables (EJW)}

The EJW outcome measures come from two different sources. First, WERS2004 monitored how satisfied employees were with eight different aspects of their job. The survey asked employees to rate - on a fivepoint scale from 'very satisfied' to 'very dissatisfied' - how satisfied they were with: (i) the sense of achievement they get from their work; (ii) the scope for using their own initiative; (iii) the amount of influence they have over their job; (iv) the training they receive; $(v)$ the amount of pay they receive; (vi) their job security; (vii) their work itself and (viii) their involvement in decision making. Secondly, WERS2004 also monitored affective well-being. Employees were asked to rate - on a five-point scale from 'all of the time' to 'Never' - how much of the time over the past few weeks they were in the following six positive and negative emotional states: (i) tense; (ii) calm; (iii) relaxed; (iv) worried; ( $v$ ) uneasy, and (vi) content.

Principal components analysis on the facets of job satisfaction identified a single factor with an eigenvalue above 1 (3.99) explaining 99\% of the variance in the eight items and with a Kaiser-Meyer-Olkin (KMO) sampling adequacy measure of 0.88 . Similarly, principal components analysis on the affective wellbeing outcomes identified one factor with an eigenvalue above 1 (3.42) explaining $88 \%$ of the variance in the six affective well-being measures and with a KMO sampling adequacy measure of 0.80 .6 Based on the principal components analyses, therefore, two different EJW measures have been generated - EJW ${ }^{1}$, which relates to employee job satisfaction, and $\mathrm{EJW}^{2}$, which relates to employee job-related affects - for the regression analysis conducted. Psychological studies emphasise the need for broader definition of work-related psychological well-being (than just job satisfaction). The vast work in this area by Warr $(1990,1994,1999)$ also justifies the use in this paper of two separate well-being measures. Affective wellbeing measures are also thought to be amongst the most important, if not the most important, measures of psychological well-being (Warr, 1994; Daniels, 2000). ${ }^{7}$ Fig. 2 in the Appendix provide plots of the two well-being outcome measures disaggregated by gender while Tables A1 and A2 report correlation coefficients between the two new measures of EJW and their respective constituent components.

\subsubsection{Diversity (WGD) and other control variables}

The $W G D$ variable of interest to this paper is defined as one minus the Herfindahl Index, using the proportion of female and male employees at each workplace as monitored in the WERS2004 establishment survey. Previous studies (e.g. Peccei and Lee, 2005) have used percentage of women in a workplace. However, a percentage measure would not capture the link between diversity and various outcome measures of interest fully. In their recent study, Leonard and Levine (2006) elucidate shortcomings of using percentage measure

\footnotetext{
${ }^{6}$ The Cronbach's alpha for the eight facets of job satisfaction and the six job-related anxiety measures are 0.85 and 0.86 , respectively. The Cronbach's alpha values are comparable to those reported in Wood (2008) and Bryson et al. (2009). On the other hand, findings from the principal components analyses are slightly different to theirs, something that is due to the use in this paper of polychoric correlation, which is more appropriate for principal component analysis involving ordinal outcomes.

${ }^{7}$ As stated earlier, the affective-wellbeing measures represent experiences of positive and negative emotional states over a short recall period ("the past few weeks") and may represent a more accurate reflection of wellbeing. In addition, job satisfaction is likely to be influenced by one's prior expectation (of, for example, a pay rise or promotion) while the affective-wellbeing measures may reflect actual feelings (of, for example, uneasiness) experienced over a short recall period, which may not be influenced by expectation as much.
}

in that it increases linearly with the size of one group of interest even though such an increase would mean a reduction in the size of another. To be able to address this issue of nonlinearity, a workplace gender diversity index, WGD, has been generated in this paper, where $W G D=1-\sum_{i} S_{k}^{2}$ and $S$ represents the shares of female and male employees at a workplace with $i=1$ and $2 .^{8}$ Fig. 1 in the Appendix depicts a plot of the WGD index generated. A number of other variables relating to employee demographic and human capital characteristics, job characteristics, industry of employment, geographic area, travel-to-work area unemployment and vacancy rates, and a range of employer characteristics are used as controls. Table A3 in the appendix reports descriptive statistics on all variables used in the empirical analysis separately for female and male employees.

\subsubsection{Workplace practice and policy summary variable}

As noted by Leonard and Levine (2006), it is vital that a study of this nature accounts for variations in workplace HRM policy and practices across workplaces. WERS2004 has gathered extensive data on workplace HRM practice and policy. Some method of reducing this vast data is essential if arbitrary choices of items are to be avoided. To this end, twelve dummy variables have been generated based on the information gathered in WERS2004regarding whether a workplace: (i) has gender equality explicitly stated in its policy, (ii) provides equal opportunities training, and (iii) monitors and reviews gender equality provisions at the workplace. Secondly, factor analysis has been conducted on the 12 dummies using orthogonal varimax principal components analysis. The analysis identifies a single factor with an eigenvalue of 3.87 explaining $40 \%$ of the variance in the selected items, forming a measure of workplace HRM policy and practice (equality) variable. The Cronbach's alpha scale reliability coefficient for the twelve HRM policy and practice dummies is 0.80 . Appendix Tables A4 and A5 report summary statistics and correlation matrix on the 'HRM equality' policy variable and the 12 dummy variables used to generate it.

\section{A framework of analysis}

\subsection{Theoretical background}

The theoretical background of relevance to this study relates to theories of discrimination where discrimination is due to either preference (Becker, 1957; Arrow, 1972, 1973; Phelps, 1972) or information (Aigner and Cain, 1977). The former stipulates that discrimination occurs when people behave as if they refuse to change their stereotypes about the capabilities of discriminated individuals or groups. It is to do with preference and may not change in the face of favourable information about the group. The information explanation, on the other hand, states that (employer) discrimination is the result of asymmetric information regarding the discriminated individual and such stereotypes alter with information. ${ }^{9}$

Akerlof and Kranton (2000) formalised the earlier "taste" based discrimination explanations by incorporating identity into a model of behaviour and showing how identity influences economic outcomes. Their formulation is based on social identity theory that posits that an individual's social identity depends on all of the identifications, including gender, the person uses in construing her/his views of the self. A person may experience anxiety when the person's internalised rules of personality (or identity or ego) are violated somehow. In the context of work, they show how identity can be related to occupation arguing "occupations are associated with the social categories 'man' and 'woman,' and individual payoffs from different types

\footnotetext{
${ }^{8}$ The diversity index is also known as Blau's Index and assumes a theoretical value ranging from a minimum of 0 , signifying perfect homogeneity, to a maximum of 0.5 , signifying perfect heterogeneity here.

9 Other theories of relevance to economists include language (difference) based discrimination (Lang, 1986) and Lazear's (1999) communication costs explanation of (racial) diversity.
} 
of work reflect these gender associations" (p. 732). Alesina and La Ferrara (2000, 2005), also formalising the social identity theory, predict that individual utility from joining a group depends positively on the share of group members of one's own type and negatively on the share of different types. The group competition theory, that was chiefly due to Blalock (1967), gives good insights into the implications of such identity formation, particularly if the size of the socially 'subordinate' group were to increase. This, according to Blalock, threatens the resource and power advantages of the 'dominant' group. In turn, such threats may prod the 'dominant' group to increase hostility and discrimination against members of the 'subordinate' group, inflicting welfare/well-being loss to the latter. ${ }^{10}$

This paper uses the social identity and group competition explanations to formulate a simple theoretical model that will be tested empirically to establish the link between WGD and EJW. ${ }^{11}$ Suppose there are two groups of workers $M$, males, and $F$, females, in a workplace with (average) well-being $E J W_{M}$ and $E J W_{F}$, respectively. Suppose also that $M$ workers are 'dominant', in terms of their group size or equally, if not more importantly, in the position they occupy in the employment hierarchy, rendering the workplace a 'man' social category. The increase in the size of $F$ workers, as reflected in the recent increase in the share of women in the labour market in Britain, may be construed as a threat to the 'dominant' $M$ workers. $M$ workers may respond to this by increasing hostility and discrimination against $F$ workers, the 'subordinate' group in this context. Two simple predictions may follow. First, if the WGD-EJW link is thought to be independent of the degree of gender mix at the workplace, one would expect the WGD-EJW relationship to take the general form;

$E J W_{F}=(1-d * W G D) E J W_{M}$

where $0<d<1$ and $W G D=1$ if there is at least one $F$ employee at the workplace with a 'man' social category and 0 otherwise. Second, if the link between WGD and EJW is thought to vary with the degree of gender mix at the workplace, then one would expect $E J W_{F}$ to decline with the degree of WGD defined in Section 3, i.e.

$E J W_{F}=f(W G D)=f\left(1-\sum_{i} S_{k}^{2}\right) ; \quad f^{\prime}<0$.

If Alesina and La Ferrara's (2000, 2005) social identity theory based prediction holds, one would expect $f^{\prime \prime}>0$. However, Blalock's notion of competition for resources, including 'power', may be more important than the sheer sizes of the $\mathrm{F}$ and $\mathrm{M}$ workers.

Based on the theoretical framework outlined, this paper puts forward the following hypotheses, which will be tested in the empirical analysis that follows:

Hypothesis 1. WGD and EJW are negatively related. This hypothesis is tested on the basis of the coefficient of the main gender diversity correlate.

Hypothesis 2. The negative relationship between WGD and EJW is associated with the 'subordinate' group, $F$ workers in this case. As outlined earlier, if $F$ workers were to experience increased hostility and discrimination from their 'threatened' $M$ counterparts, this would result in a reduction in $E J W_{F}$. This is tested on the basis of gender based sub-group analysis.

Hypothesis 3. Alesina and La Ferrara's social identity theory based stipulation means the EJW of women increases with the size of F workers. However, the workplace power structure will also be equally, if not

\footnotetext{
10 Blalock (1967) defines resources as "the actual sources of power or those properties of the individual or group that provide the power potential or ability to exercise power" (p. 113).

${ }^{11}$ In contrast to the earlier (taste-based discrimination) explanation, which is primarily aimed at explaining racial discrimination, these explanations lend the scope for analysing gender related outcomes, including gender discrimination.
}

more, important as Blalock notes. This is tested on the basis of an interaction term between WGD and a female majority dummy.

Hypothesis 4. The presence of HRM policy and practices that promote gender equality may help curtail adverse well-being effects stemming from the way $M$ workers respond to the 'threat' from $F$ workers. This is tested based on the HRM policy and practices variable. One would expect gender equality centred initiatives to eliminate negative association, if any, between WGD and EJW.

\subsection{Empirical methodology}

The empirical models estimated in this paper are based on the utility function formulation, where self-reported EJW proxies the level of utility, $U$, a worker derives from their work/workplace. EJW is influenced by factors related to the employee and their workplace, including the level of WGD, and can be given by;

$E J W_{i j}^{k}=f\left(W G D_{j}, \mathbf{E E}_{i j}, \mathbf{E R}_{j}\right)+\varepsilon_{i j}, \quad k=1,2 ; \quad i=1, \ldots, I$ and $j=1, \ldots, J$

where, EE stands for the vector of employee demographic, human capital and job related characteristics; ER stands for the vector of workplace related characteristics; $i, j$ and $k$ signify employees, workplaces and type of EJW respectively. As detailed in Section 3.2, the paper uses two different measures of EJW for each employee $i$ in a workplace $j, E J W_{i j}^{k}$, which are based on self-reported well-being that represent underlying continuous latent measures of well-being, EJW $W_{i j}^{*}$.

Eq. (3) is estimated using OLS. ${ }^{12}$ However, the non-experimental nature of the WERS study makes it essential that unobserved heterogeneity is accounted for. The level of WGD or the particular HRM policy and practices observed at a workplace are unlikely to represent random phenomena, given possible employer and employee selection. To the extent that there is such selection, addressing the issue of non-randomness becomes crucial to avoid the potentially biassing selection effects. This paper attempts to account for such potential biases by estimating random effects models that exploit the nested structure of the WERS2004 data. As noted in Section 3, there are at least two employees per workplace in the data allowing controlling for workplace-level unobserved heterogeneity as,

$E J W_{i j}^{k}=f\left(W G D_{j}, \mathbf{E E}_{i j}, \mathbf{E R}_{j}\right)+\alpha_{j}+\varepsilon_{i j}, \quad k=1,2 ; \quad i=1, \ldots, I ; \quad j=1, \ldots, J$

where $\alpha_{j}$ are unobserved workplace effects assumed to be random and to follow a probability distribution known up to some finite set of parameters. ${ }^{13}$ Comparing estimated parameters obtained from Eq. (4) with that of parameters from OLS with a cluster option permits establishing if unobserved workplace effects makes a difference.

\section{Empirical results and discussion}

Figs. 3 and 4 in the Appendix depict locally weighted lowess smoothers for the two well-being outcome measures of interest. Fig. 3

\footnotetext{
12 This is done with the cluster option to account for the presence of at least two employees from same workplace.

${ }^{13}$ Orthogonality of $\alpha_{j}$ is a strong assumption since the workplace random effects may well be correlated with the regressors, particularly the workplace specific ones. This may be so if, for example, unmeasured attributes of the workplace such as commitment or motivation of the manager were to influence the workplace-level (or, for that matter employee-level) correlates, including WGD. That the paper uses a battery of employee and workplace correlates that include a measure of HRM policy and practices may mitigate this problem to some degree however. Estimating (workplace) FE regression cannot be an option given multicollinearity between the workplace fixed effect and the measure of WGD. Also, it is not possible to account for employee-level unobserved heterogeneity using WERS, since WERS does not re-survey the same employees.
} 
Table 1

Main effect, WGD and job satisfaction.

\begin{tabular}{|c|c|c|c|c|c|c|}
\hline & OLS & $\mathrm{RE}$ & OLS & $\mathrm{RE}$ & OLS & RE \\
\hline Gender diversity & $\begin{array}{l}-0.262^{\text {**** }} \\
(0.044)\end{array}$ & $\begin{array}{l}-0.280^{* * *} \\
(0.061)\end{array}$ & $\begin{array}{l}-0.108^{* *} \\
(0.046)\end{array}$ & $\begin{array}{l}-0.130^{* *} \\
(0.062)\end{array}$ & $\begin{array}{l}-0.095^{* *} \\
(0.047)\end{array}$ & $\begin{array}{l}-0.113^{*} \\
(0.062)\end{array}$ \\
\hline Gender equality policy and practice & & & & & $\begin{array}{l}-0.016^{* *} \\
(0.008)\end{array}$ & $\begin{array}{l}-0.022^{* *} \\
(0.011)\end{array}$ \\
\hline Constant & $\begin{array}{l}4.537^{* * *} \\
(0.053)\end{array}$ & $\begin{array}{l}4.560^{* * *} \\
(0.055)\end{array}$ & $\begin{array}{l}4.492^{* * * *} \\
(0.064)\end{array}$ & $\begin{array}{l}4.513^{* * *} \\
(0.073)\end{array}$ & $\begin{array}{l}4.492^{* * *} \\
(0.064)\end{array}$ & $\begin{array}{l}4.511^{* * *} \\
(0.073)\end{array}$ \\
\hline No. of employees & 18,064 & 18,064 & 18,064 & 18,064 & 18,064 & 18,064 \\
\hline R-squared & 0.091 & & 0.109 & & 0.110 & \\
\hline No. of workplaces & & 1506 & & 1506 & & 1506 \\
\hline
\end{tabular}

Robust standard errors in parentheses.

$* * * \quad \mathrm{p}<0.01$.

** $\mathrm{p}<0.05$.

$* \mathrm{p}<0.1$.

shows a decline in job satisfaction $\left(E J W^{1}\right)$ for women as WGD increases. On the other hand, the lowess smoother for men shows an increase in job satisfaction with WGD, though the increase appears to be marginal. The picture is different for job-related affective well-being $\left(E J W^{2}\right)$, where more or less similar patterns emerge for female and male employees. The lowess smoothers do not control for other influences, something the regression based results reported in Tables 1-6 below do.

The regression analysis conducted involves three different specifications. The first specification controls for extensive employee characteristics, while the second specification controls for extensive set of employer characteristics in addition. The final specification makes a further control on workplace HRM policy and practices. Each of the three specifications has also been modelled using OLS and RE regressions. The use of these specifications helps establish if accounting for extensive sets of observable employee and employer characteristics makes a difference in explaining the link between WGD and EJW, while the RE specification also allows determining if unobservable workplace characteristics are worth taking into account.

\subsection{WGD and job satisfaction (EJW' $\left.{ }^{1}\right)$}

Tables 1-3 report estimation results pertaining to job satisfaction. Results reported in Table 1 indicate the main effect of WGD on job satisfaction, which is negative and statistically significant. Accounting for workplace characteristics, including HRM policy and practices, do reduce the negative effect both in magnitude and statistical significance; but they do not eliminate the negative effect entirely however. As can be seen from the final column, Table 1, WGD reduces employee job satisfaction by 11.3 percentage points at the $10 \%$ level of significance. Remarkably, the coefficient of the HRM policy and practice variable is itself negative and statistically significant at the conventional level of significance. These results lend support to Hypothesis 1 but reject Hypothesis 4.

Table 2 reports estimates from the gender-based sub-group analysis undertaken to determine if the negative link between WGD and EJW relates to female employees as Hypothesis 2 stipulates. This is indeed found to be the case as shown by the results in Table 2 . As before, controlling for extensive sets of employee and workplace characteristics, including HRM policy and practices, reduces this negative effect but does not eliminate it entirely. As can be seen from the final column in Table 2, WGD is associated with a 27.3 percentage point reduction in job satisfaction for women. The presence of gender centred HRM policy and practice at the workplace is again found to be negatively association with EJW, contrary to expectation and Hypothesis 4. In contrast, no statistically significant link is found for male employees, as the lower panel of Table 2 reports. The HRM policy and practices variable is also found to be statistically insignificant for males.

Table 3 reports findings that lend support to Hypothesis 3 that relate to group size related influences. For the purpose an interaction term of the dummy variable, which assumes a value 1 if female employees are a majority at workplaces (>50\%), and the WGD variable has been used to determine the link between WGD and EJW. ${ }^{14}$ This variable is then interacted with WGD to determine what the effect of WGD might be in settings where women have the numerical majority. The findings reported in Table 3 suggest that even in settings where female employees are the majority, WGD is found to be negatively and significantly related to job satisfaction. This may be the result of 'competition for resources and power' Blalock notes, which is likely to get more intense if the 'subordinate' group has the numerical majority. If, as has been witnessed in the past few decades, more and more women come to the labour market, but do not occupy higher echelons of the employment hierarchy, this could lead to such outcomes.

Occupying higher positions in the employment hierarch is likely to require long and uninterrupted tenure and/or employment, something that may not be as straightforward for females as it may be for their male counterparts, given that (most) women have interrupted careers. This can have two potential effects. First, as Blalock argues the sheer size of female employees and the prospect that some out of the 'majority' may someday make it through to the top hierarchy may threaten the 'resource and power advantage' men may still command, even where they are minority numerically. Such threat may prod increased hostility from male employees resulting in reduced job satisfaction for females. Secondly, their numerical majority status may offer women a better opportunity to 'face up' to male colleagues who, in some cases, might have gone further up in the employment hierarchy while some of their female counterparts have taken a career break for maternity reasons. ${ }^{15}$ The likely outcome of both of these is to increase 'hostility' at the workplace, which is likely to have adverse job satisfaction outcomes. Remarkably, the WGD coefficient in Table 3 is statistically significant and suggests a positive link between WGD and job satisfaction where females are a numerical minority (or males a numerical major), a finding that is robust at conventional level of significance.

\subsection{WGD and job-related affective well-being $\left(E J W^{2}\right)$}

Tables 4-6 report estimation results pertaining to affective wellbeing. Table 4 reports the main effect of WGD on job-related affective wellbeing, which shows negative and statistically significant association between WGD and job satisfaction. Accounting for workplace characteristics and HRM policy and practices do again reduce the negative effect both in magnitude and statistical significance. They do not eliminate the negative effect altogether however, as can be seen from the final column of Table 4, where WGD reduces employees' affective well-being by 11.5 percentage points at $10 \%$ level of significance. The

\footnotetext{
${ }^{14}$ The 'majority female' variable has been included in all of the specifications as an independent regressor to be able to account for the asymmetry nature of the WGD variable.

15 The idea here is along the union-voice argument of Freeman and Medoff (1984) where some discontented female employees might use gender comradery and exploit their numerical majority 'advantage' to mobilise or encourage confrontation to advance own (and women's) demands.
} 
Table 2

WGD and job satisfaction, gender-based sub-groups.

\begin{tabular}{|c|c|c|c|c|c|c|}
\hline & OLS & $\mathrm{RE}$ & OLS & $\mathrm{RE}$ & OLS & $\mathrm{RE}$ \\
\hline \multicolumn{7}{|l|}{ Female } \\
\hline Gender diversity & $\begin{array}{l}-0.496^{* * *} \\
(0.057)\end{array}$ & $\begin{array}{l}-0.493^{* * *} \\
(0.077)\end{array}$ & $\begin{array}{l}-0.287^{* * *} \\
(0.062)\end{array}$ & $\begin{array}{l}-0.295^{* * * *} \\
(0.079)\end{array}$ & $\begin{array}{l}-0.263^{* * *} \\
(0.063)\end{array}$ & $\begin{array}{l}-0.273^{\text {*** }} \\
(0.080)\end{array}$ \\
\hline Gender equality policy and practice & & & & & $\begin{array}{l}-0.023^{* *} \\
(0.010)\end{array}$ & $\begin{array}{l}-0.025^{*} \\
(0.013)\end{array}$ \\
\hline Constant & $\begin{array}{l}4.630^{* * *} \\
(0.071)\end{array}$ & $\begin{array}{l}4.642^{* * *} \\
(0.075)\end{array}$ & $\begin{array}{l}4.516^{\text {*** }} \\
(0.087)\end{array}$ & $\begin{array}{l}4.566^{\text {*** }} \\
(0.097)\end{array}$ & $\begin{array}{l}4.516^{* * *} \\
(0.087)\end{array}$ & $\begin{array}{l}4.564^{* * *} \\
(0.097)\end{array}$ \\
\hline No. of employees & 9450 & 9450 & 9450 & 9450 & 9450 & 9450 \\
\hline R-squared & 0.086 & & 0.106 & & 0.106 & \\
\hline No. of workplaces & & 1409 & & 1409 & & 1409 \\
\hline \multicolumn{7}{|l|}{ Male } \\
\hline Gender diversity & $\begin{array}{l}0.061 \\
(0.077)\end{array}$ & $\begin{array}{l}0.013 \\
(0.096)\end{array}$ & $\begin{array}{l}0.102 \\
(0.079)\end{array}$ & $\begin{array}{l}0.074 \\
(0.097)\end{array}$ & $\begin{array}{l}0.105 \\
(0.080)\end{array}$ & $\begin{array}{l}0.082 \\
(0.098)\end{array}$ \\
\hline Gender equality policy and practice & & & & & $\begin{array}{l}-0.004 \\
(0.012)\end{array}$ & $\begin{array}{l}-0.012 \\
(0.015)\end{array}$ \\
\hline Constant & $\begin{array}{l}4.518^{* * *} \\
(0.084)\end{array}$ & $\begin{array}{l}4.543^{* * *} \\
(0.086)\end{array}$ & $\begin{array}{l}4.526^{\text {*** }} \\
(0.100)\end{array}$ & $\begin{array}{l}4.531^{\text {*** }} \\
(0.110)\end{array}$ & $\begin{array}{l}4.526^{\text {*** }} \\
(0.100)\end{array}$ & $\begin{array}{l}4.530^{* * *} \\
(0.110)\end{array}$ \\
\hline No. of employees & 8614 & 8614 & 8614 & 8614 & 8614 & 8614 \\
\hline R-squared & 0.101 & & 0.118 & & 0.118 & \\
\hline No. of workplaces & & 1336 & & 1336 & & 1336 \\
\hline
\end{tabular}

Robust standard errors in parentheses.

$$
\begin{array}{rl}
* * * & \mathrm{p}<0.01 . \\
* * & \mathrm{p}<0.05 . \\
* & \mathrm{p}<0.1 .
\end{array}
$$

coefficient of the HRM policy and practice variable is negative for both OLS and RE specifications but statistically insignificant in the latter case. In all, the evidence does lend some support to Hypothesis 1, albeit weakly, but does fail to lend support in favour or against Hypothesis 4.

Table 5 reports results from gender-based sub-group analyses. The coefficient of the WGD correlate is negative for the women sub-group in all cases but statistically insignificant in the richer specifications, which control for workplace characteristics. This may underscore the need for controlling for both employee and employer characteristics extensively. For the sub-group of male employees, the WGD coefficients are negative in nearly all cases confirming the pattern exhibited in Fig. 4; but none of these is found to be statistically significant. HRM policy and practice is also not found to have statistically significant association with affective well-being both for female and male sub-groups. Once again, the evidence obtained offers at best a weaker support to Hypothesis 2 .

Table 6 reports parameter estimates that test the hypothesis on group size related influences. None of the coefficient estimates reported was found to be statistically significant, unlike the findings for job satisfaction reported in Table 3 above. The coefficient of the HRM policy and practice variable is negative in both specifications but statistically insignificant for the RE specification. Once again, the results reported in Table 6 fail to lend support to Hypotheses 3 and 4.

\section{Summary and conclusion}

This paper attempted to establish empirically the link between WGD and EJW using the WERS2004 data. It uses an index measure of WGD and two different measures of EJW obtained from the eight facets job satisfaction and the six measures of affective well-being monitored in WERS2004. The paper made extensive review of the relevant theoretical and empirical literatures before setting up four testable hypotheses regarding: (i) the link between WGD and EJW, (ii) whether the link is gender specific, (iii) if the size of a gender group may have influences on the WGD and EJW link, and (iv) whether workplace HRM policy and practice has any bearing on the link between WGD and EJW. The empirical analysis undertaken involves several specifications that are meant to test for robustness of the statistical estimates. The paper also employed RE model as one of the specifications to determine whether accounting for unobserved workplace heterogeneity makes a difference, something previous UK studies on gender composition ignore.

The empirical analysis undertaken attempted to test the four hypotheses regarding the link between WGD and EJW using both measures of EJW. As detailed in Section 5, the findings in the paper lend strong support (positive and negative) to each of the four hypotheses with respect to the job satisfaction well-being outcome. On the other hand, there is

\begin{tabular}{|c|c|c|c|c|c|c|}
\hline & OLS & RE & OLS & RE & OLS & RE \\
\hline Gender diversity & $\begin{array}{l}0.164^{* *} \\
(0.071)\end{array}$ & $\begin{array}{l}0.179^{*} \\
(0.093)\end{array}$ & $\begin{array}{l}0.188^{* *} \\
(0.073)\end{array}$ & $\begin{array}{l}0.187^{\text {** }} \\
(0.094)\end{array}$ & $\begin{array}{l}0.193^{* * *} \\
(0.073)\end{array}$ & $\begin{array}{l}0.196^{* *} \\
(0.094)\end{array}$ \\
\hline Gender diversity $*$ Majority female & $\begin{array}{l}-0.766^{* * *} \\
(0.093)\end{array}$ & $\begin{array}{l}-0.813^{\text {*** }} \\
(0.125)\end{array}$ & $\begin{array}{l}-0.558^{* * *} \\
(0.100)\end{array}$ & $\begin{array}{l}-0.587^{* * *} \\
(0.130)\end{array}$ & $\begin{array}{l}-0.547^{* * *} \\
(0.100)\end{array}$ & $\begin{array}{l}-0.574^{\text {*** }} \\
(0.130)\end{array}$ \\
\hline Gender equality policy and practice & & & & & $\begin{array}{l}-0.014^{*} \\
(0.008)\end{array}$ & $\begin{array}{l}-0.020^{*} \\
(0.011)\end{array}$ \\
\hline Constant & $\begin{array}{l}4.393^{\text {*** }} \\
(0.056)\end{array}$ & $\begin{array}{l}4.410^{* * *} \\
(0.060)\end{array}$ & $\begin{array}{l}4.383^{\text {*** }} \\
(0.067)\end{array}$ & $\begin{array}{l}4.397^{\text {*** }} \\
(0.078)\end{array}$ & $\begin{array}{l}4.385^{\text {*** }} \\
(0.067)\end{array}$ & $\begin{array}{l}4.397^{\text {*** }} \\
(0.078)\end{array}$ \\
\hline No. of employees & 18,064 & 18,064 & 18,064 & 18,064 & 18,064 & 18,064 \\
\hline R-squared & 0.094 & & 0.111 & & 0.111 & \\
\hline No. of workplaces & & 1506 & & 1506 & & 1506 \\
\hline
\end{tabular}

Table 3

WGD and job satisfaction, group size related link.

Robust standard errors in parentheses.

** $\mathrm{p}<0.05$.

$* \mathrm{p}<0.1$.

$* * * \quad \mathrm{p}<0.01$ 
Table 4

WGD and job-related affective well-being, main effect.

\begin{tabular}{|c|c|c|c|c|c|c|}
\hline & OLS & $\mathrm{RE}$ & OLS & RE & OLS & RE \\
\hline Gender diversity & $\begin{array}{l}-0.122^{* *} \\
(0.048)\end{array}$ & $\begin{array}{l}-0.146^{* *} \\
(0.060)\end{array}$ & $\begin{array}{l}-0.106^{* *} \\
(0.051)\end{array}$ & $\begin{array}{l}-0.129^{* *} \\
(0.062)\end{array}$ & $\begin{array}{l}-0.095^{*} \\
(0.051)\end{array}$ & $\begin{array}{l}-0.115^{*} \\
(0.063)\end{array}$ \\
\hline Gender equality policy and practice & & & & & $\begin{array}{l}-0.015^{*} \\
(0.008)\end{array}$ & $\begin{array}{l}-0.017 \\
(0.011)\end{array}$ \\
\hline Constant & $\begin{array}{l}3.961^{\text {*** }} \\
(0.057)\end{array}$ & $\begin{array}{l}3.956^{\text {*** }} \\
(0.060)\end{array}$ & $\begin{array}{l}3.955^{\text {*** }} \\
(0.070)\end{array}$ & $\begin{array}{l}3.955^{\text {**** }} \\
(0.078)\end{array}$ & $\begin{array}{l}3.955^{\text {*** }} \\
(0.070)\end{array}$ & $\begin{array}{l}3.954^{* * *} \\
(0.078)\end{array}$ \\
\hline No. of employees & 18,064 & 18,064 & 18,064 & 18,064 & 18,064 & 18,064 \\
\hline R-squared & 0.090 & & 0.095 & & 0.095 & \\
\hline No. of workplaces & & 1506 & & 1506 & & 1506 \\
\hline
\end{tabular}

Robust standard errors in parentheses.

$* * \mathrm{p}<0.05$.

* $\mathrm{p}<0.1$.

$* * * \mathrm{p}<0.01$

Table 5

WGD and job-related affective well-being, gender-based sub-groups.

\begin{tabular}{|c|c|c|c|c|c|c|}
\hline & OLS & $\mathrm{RE}$ & OLS & $\mathrm{RE}$ & OLS & $\mathrm{RE}$ \\
\hline \multicolumn{7}{|l|}{ Female } \\
\hline Gender diversity & $\begin{array}{l}-0.155^{* *} \\
(0.065)\end{array}$ & $\begin{array}{l}-0.148^{*} \\
(0.082)\end{array}$ & $\begin{array}{l}-0.122^{*} \\
(0.071)\end{array}$ & $\begin{array}{c}-0.116 \\
(0.087)\end{array}$ & $\begin{array}{l}-0.104 \\
(0.072)\end{array}$ & $\begin{array}{c}-0.097 \\
(0.088)\end{array}$ \\
\hline Gender equality policy and practice & & & & & $\begin{array}{l}-0.017 \\
(0.011)\end{array}$ & $\begin{array}{l}-0.021 \\
(0.014)\end{array}$ \\
\hline Constant & $\begin{array}{l}3.944^{\text {*** }} \\
(0.079)\end{array}$ & $\begin{array}{l}3.933^{* * *} \\
(0.084)\end{array}$ & $\begin{array}{l}3.929^{* * *} \\
(0.097)\end{array}$ & $\begin{array}{l}3.954^{\text {*** }} \\
(0.109)\end{array}$ & $\begin{array}{l}3.929^{* * *} \\
(0.097)\end{array}$ & $\begin{array}{l}3.952^{* * *} \\
(0.109)\end{array}$ \\
\hline No. of employees & 9450 & 9450 & 9450 & 9450 & 9450 & 9450 \\
\hline R-squared & 0.108 & & 0.114 & & 0.115 & \\
\hline No. of workplaces & & 1409 & & 1409 & & 1409 \\
\hline \multicolumn{7}{|l|}{ Male } \\
\hline Gender diversity & $\begin{array}{l}-0.031 \\
(0.080)\end{array}$ & $\begin{array}{l}-0.052 \\
(0.092)\end{array}$ & $\begin{array}{l}-0.009 \\
(0.083)\end{array}$ & $\begin{array}{l}-0.036 \\
(0.095)\end{array}$ & $\begin{array}{l}0.000 \\
(0.084)\end{array}$ & $\begin{array}{l}-0.025 \\
(0.095)\end{array}$ \\
\hline Gender equality policy and practice & & & & & $\begin{array}{l}-0.015 \\
(0.012)\end{array}$ & $\begin{array}{l}-0.016 \\
(0.015)\end{array}$ \\
\hline Constant & $\begin{array}{l}3.876^{\text {*** }} \\
(0.087)\end{array}$ & $\begin{array}{l}3.864^{\text {*** }} \\
(0.090)\end{array}$ & $\begin{array}{l}3.889^{* * *} \\
(0.106)\end{array}$ & $\begin{array}{l}3.873^{\text {*** }} \\
(0.112)\end{array}$ & $\begin{array}{l}3.888^{\text {*** }} \\
(0.106)\end{array}$ & $\begin{array}{l}3.872^{\text {*** }} \\
(0.112)\end{array}$ \\
\hline No. of employees & 8614 & 8614 & 8614 & 8614 & 8614 & 8614 \\
\hline R-squared & 0.074 & & 0.079 & & 0.080 & \\
\hline No. of workplaces & & 1336 & & 1336 & & 1336 \\
\hline
\end{tabular}

Robust standard errors in parentheses.

** $\mathrm{p}<0.05$.

$* \mathrm{p}<0.1$.

$* * * \mathrm{p}<0.01$.

Table 6

WGD and job-related affective well-being, group size related link.

\begin{tabular}{|c|c|c|c|c|c|c|}
\hline & OLS & $\mathrm{RE}$ & OLS & $\mathrm{RE}$ & OLS & $\mathrm{RE}$ \\
\hline Gender diversity & $\begin{array}{c}-0.099 \\
(0.076)\end{array}$ & $\begin{array}{l}-0.114 \\
(0.092)\end{array}$ & $\begin{array}{l}-0.069 \\
(0.079)\end{array}$ & $\begin{array}{l}-0.092 \\
(0.095)\end{array}$ & $\begin{array}{l}-0.063 \\
(0.079)\end{array}$ & $\begin{array}{l}-0.084 \\
(0.095)\end{array}$ \\
\hline Gender diversity*Majority female & $\begin{array}{l}-0.040 \\
(0.102)\end{array}$ & $\begin{array}{l}-0.056 \\
(0.125)\end{array}$ & $\begin{array}{l}-0.071 \\
(0.110)\end{array}$ & $\begin{array}{c}-0.069 \\
(0.133)\end{array}$ & $\begin{array}{l}-0.059 \\
(0.110)\end{array}$ & $\begin{array}{l}-0.058 \\
(0.133)\end{array}$ \\
\hline Gender equality policy and practice & & & & & $\begin{array}{l}-0.015^{*} \\
(0.008)\end{array}$ & $\begin{array}{l}-0.017 \\
(0.011)\end{array}$ \\
\hline Constant & $\begin{array}{l}3.954^{\text {*** }} \\
(0.060)\end{array}$ & $\begin{array}{l}3.946^{\text {*** }} \\
(0.064)\end{array}$ & $\begin{array}{l}3.941^{\text {*** }} \\
(0.073)\end{array}$ & $\begin{array}{l}3.941^{\text {*** }} \\
(0.082)\end{array}$ & $\begin{array}{l}3.944^{\text {*** }} \\
(0.073)\end{array}$ & $\begin{array}{l}3.942^{* * *} \\
(0.082)\end{array}$ \\
\hline No. of employees & 18,064 & 18,064 & 18,064 & 18,064 & 18,064 & 18,064 \\
\hline R-squared & 0.090 & & 0.095 & & 0.095 & \\
\hline No. of workplaces & & 1506 & & 1506 & & 1506 \\
\hline
\end{tabular}

Robust standard errors in parentheses.

$$
\begin{array}{r}
* \mathrm{p}<0.1 . \\
* * \mathrm{p}<0.05 . \\
* * * \mathrm{p}<0.01 .
\end{array}
$$


very limited evidence in support of the hypotheses with regards to the job-related affective well-being outcomes. Accordingly, WGD and job satisfaction are found to be negatively and significantly related in each of the models estimated. Sub-group based findings clearly indicate that the negative association between WGD and job satisfaction is specific to women. The findings also suggest group-size related link between WGD and job satisfaction in that where female employees are a numerical majority, the negative association between WGD and job satisfaction becomes stronger and highly significant. In contrast, where female employees are a numerical minority (or where male employees are a numerical majority) there is a positive and statistically significant link between WGD and job satisfaction. These group-size related links are in line with Blalock's 'group competition explanation' based predictions.

As noted at the outset of this paper, the share of women in the labour market in the UK has increased considerably in recent decades. This has happened in the face of widespread gender discrimination reported in the literature. In such a context, gender diversity may lead to workplace hostility that may affect workplace well-being adversely. Given the well-established link between job satisfaction and labour market attachment (quit and absenteeism primarily), the increase in WGD may have some serious cost implications for employees, employers and society at large. This may be so especially if the increase in WGD is not matched by active policies promoting gender equality. ${ }^{16}$

It is important to note here the value of controlling for extensive sets of employee- and employer-related influences, including the unobserved ones, in a study of this nature. As the evidence in this paper shows, not accounting for employer characteristics and failing to account for unobserved influences do make a difference. Still some level of caution has to be exercised in the interpretation of the findings in this paper however. Although the empirical analysis undertaken makes extensive control on observable employee and employer characteristics, as well as accounting for unmeasured workplace-level influences, it is impossible to rule out outstanding issues of endogeneity. A study that uses panel data will, for example, be better placed to address such concerns more fully. Regardless, the findings in this paper do suggest patterns worthy of the attention of workplace policy makers and researchers alike.

\section{Acknowledgements}

The author is grateful to the Economic and Social Research Council for the research grant RES-000-22-2465.

The author also acknowledges the Department of Trade and Industry, the Economic and Social Research Council, the Advisory, Conciliation and Arbitration Service and the Policy Studies Institute as the originators of the 2004 Workplace Employment Relations Survey data, and the Data Archive at the University of Essex as the distributor of the data. The National Centre for Social Research was commissioned to conduct the survey fieldwork on behalf of the sponsors. None of these organisations bears any responsibility for the author's analysis and interpretations of the data.

The paper benefited from feedback by Michael White, Alex Bryson and Helen Bewley on an earlier draft and excellent research assistance by Hirut Mengistu. Early findings were presented at the 2008 WERS-WPEG meeting in Sheffield, the 2008 SOLE conference as well as seminars at PSI, DWP, WBS, Cardiff and Mannheim. The author would like to thank the participants at these events for useful comments. The usual disclaimer applies.

\footnotetext{
${ }^{16}$ For example, Kochan et al. (2003) report adverse effects of racial diversity on team processes being mitigated through training and development-focused initiatives.
}

\section{Appendix A. Figures}

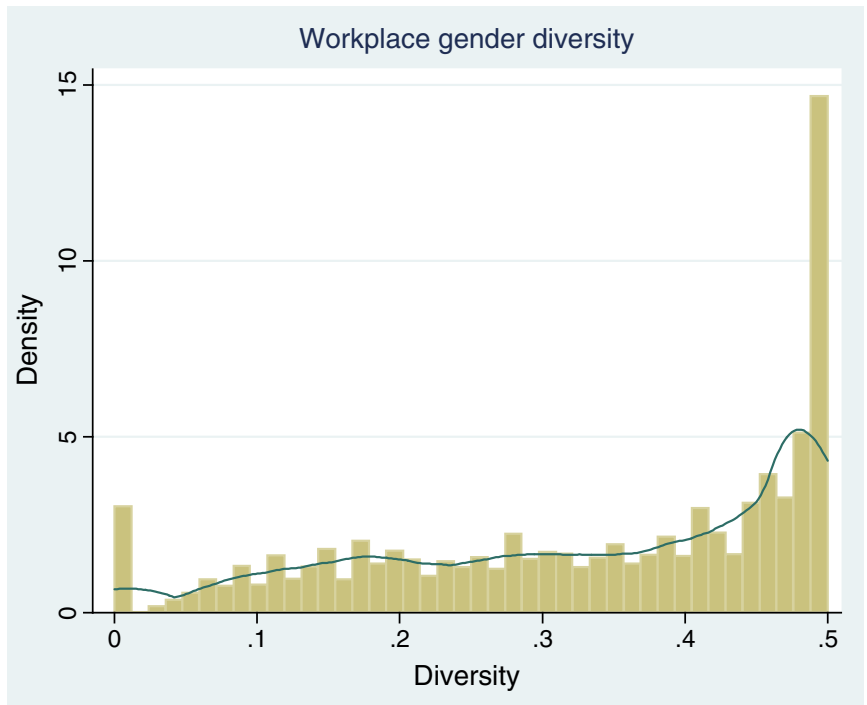

Fig. 1. Workplace gender diversity.
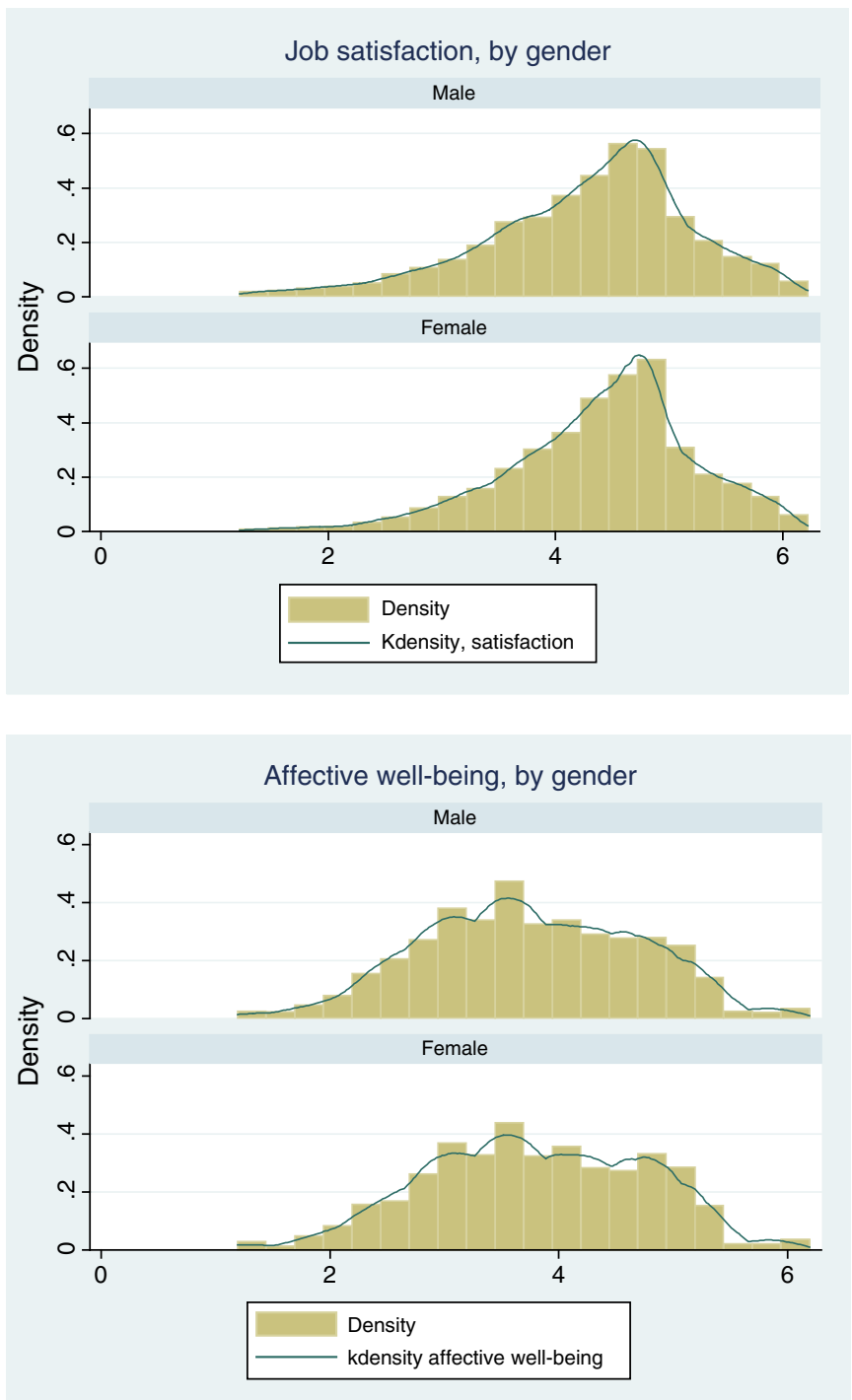

Fig. 2. Plots of EJW by gender 

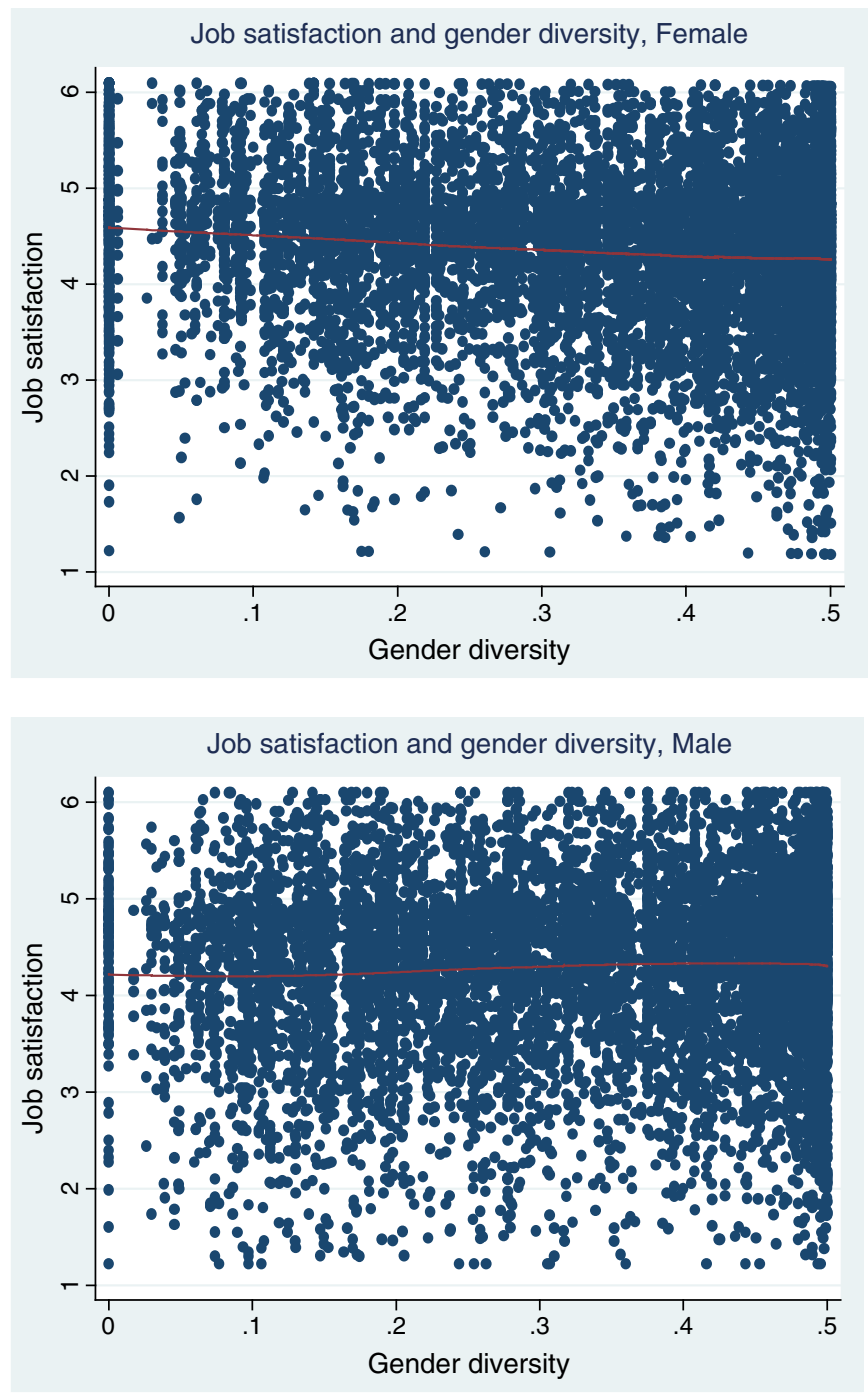

Fig. 3. Lowess smoother of job satisfaction and gender diversity.

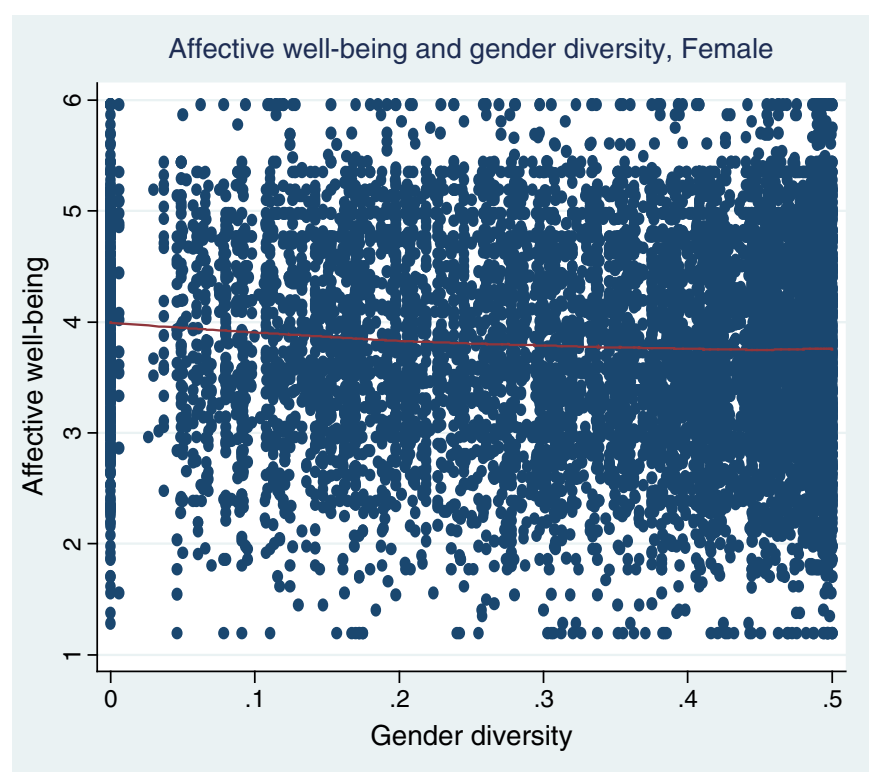

Affective well-being and gender diversity, Male

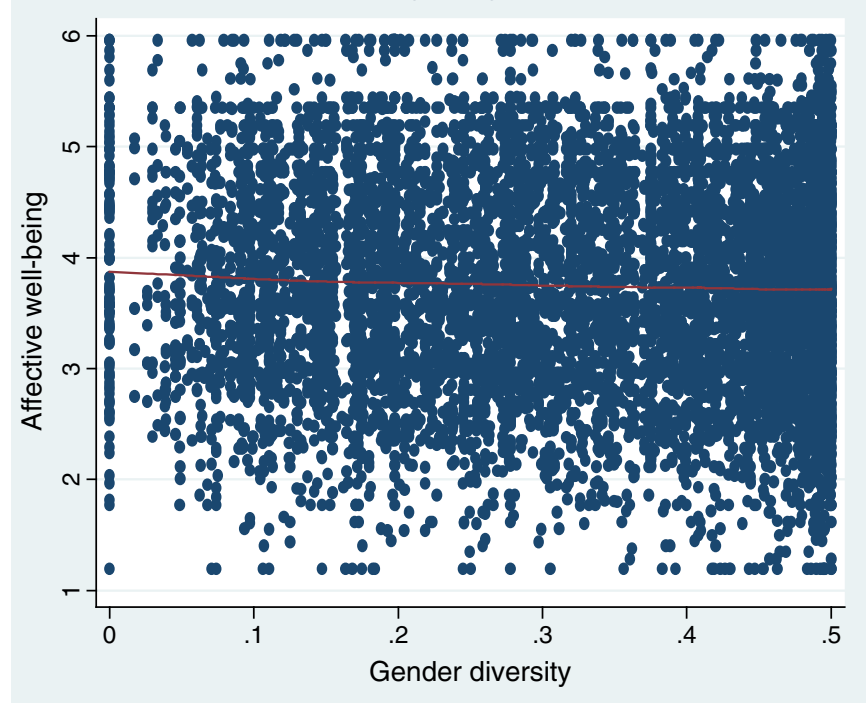

Fig. 4. Lowess smoother affective well-being and gender diversity, by gender.

Appendix A. Tables - descriptive statistics and full regression outputs

Table A1

Correlation matrix, job satisfaction outcome and its constituent components of facets of job satisfaction $(\mathrm{N}=18,064)$.

\begin{tabular}{|c|c|c|c|c|c|c|c|c|c|}
\hline & JOB SATIS & Achieve & Initiative & Influence & Training & Pay & Job-security & work itself & Decision \\
\hline JOB SATISFACTION & 1.000 & & & & & & & & \\
\hline Achievement & 0.813 & 1.000 & & & & & & & \\
\hline Initiative & 0.833 & 0.639 & 1.000 & & & & & & \\
\hline Influence & 0.852 & 0.589 & 0.726 & 1.000 & & & & & \\
\hline Training & 0.593 & 0.382 & 0.376 & 0.416 & 1.000 & & & & \\
\hline Pay & 0.482 & 0.274 & 0.278 & 0.318 & 0.335 & 1.000 & & & \\
\hline Job security & 0.525 & 0.331 & 0.317 & 0.361 & 0.363 & 0.305 & 1.000 & & \\
\hline Work itself & 0.762 & 0.681 & 0.546 & 0.535 & 0.364 & 0.284 & 0.350 & 1.000 & \\
\hline Decision making & 0.693 & 0.438 & 0.496 & 0.557 & 0.415 & 0.366 & 0.344 & 0.423 & 1.000 \\
\hline
\end{tabular}


Table A2

Correlation matrix, affective well-being and its constituent components of positive and negative affects $(\mathrm{N}=18,064)$.

\begin{tabular}{|c|c|c|c|c|c|c|c|}
\hline & AFFECT & Tense & Calm & Relaxed & Worried & Uneasy & Content \\
\hline AFFECT & 1.000 & & & & & & \\
\hline Tense & 0.758 & 1.000 & & & & & \\
\hline Calm & 0.832 & 0.490 & 1.000 & & & & \\
\hline Relaxed & 0.833 & 0.492 & 0.791 & 1.000 & & & \\
\hline Worried & 0.733 & 0.638 & 0.388 & 0.379 & 1.000 & & \\
\hline Uneasy & 0.730 & 0.589 & 0.385 & 0.371 & 0.706 & 1.000 & \\
\hline Content & 0.652 & 0.383 & 0.575 & 0.581 & 0.301 & 0.374 & 1.000 \\
\hline
\end{tabular}

Table A3

Descriptive statistics on all control variables, by Gender.

\begin{tabular}{|c|c|c|c|c|c|c|c|c|}
\hline \multirow[t]{2}{*}{ Variable } & \multicolumn{4}{|l|}{ Female } & \multicolumn{4}{|l|}{ Male } \\
\hline & Mean & Std. dev. & Min & Max & Mean & Std. dev. & Min & Max \\
\hline Gender diversity & 0.3350 & 0.1537 & 0 & 0.5 & 0.3303 & 0.1448 & 0 & 0.5 \\
\hline Majority female (female > 50\%) & 0.7527 & 0.4315 & 0 & 1 & 0.2797 & 0.4489 & 0 & 1 \\
\hline Age $<30$ & 0.2314 & 0.4218 & 0 & 1 & 0.2000 & 0.4000 & 0 & 1 \\
\hline Age $30-39$ & 0.2519 & 0.4341 & 0 & 1 & 0.2604 & 0.4389 & 0 & 1 \\
\hline Age $50+$ & 0.2430 & 0.4289 & 0 & 1 & 0.2749 & 0.4465 & 0 & 1 \\
\hline Married & 0.6694 & 0.4704 & 0 & 1 & 0.6989 & 0.4588 & 0 & 1 \\
\hline White & 0.9479 & 0.2222 & 0 & 1 & 0.9505 & 0.2168 & 0 & 1 \\
\hline Children $<7$ year old & 0.1551 & 0.3620 & 0 & 1 & 0.2098 & 0.4072 & 0 & 1 \\
\hline Other dependents & 0.1803 & 0.3845 & 0 & 1 & 0.1376 & 0.3445 & 0 & 1 \\
\hline Disabled & 0.1078 & 0.3102 & 0 & 1 & 0.1304 & 0.3367 & 0 & 1 \\
\hline No academic qualification & 0.1273 & 0.3333 & 0 & 1 & 0.1665 & 0.3725 & 0 & 1 \\
\hline O-level & 0.2455 & 0.4304 & 0 & 1 & 0.2144 & 0.4104 & 0 & 1 \\
\hline A-level & 0.0951 & 0.2934 & 0 & 1 & 0.0875 & 0.2826 & 0 & 1 \\
\hline Other qualification & 0.3599 & 0.4800 & 0 & 1 & 0.3172 & 0.4654 & 0 & 1 \\
\hline On permanent contract & 0.9139 & 0.2806 & 0 & 1 & 0.9324 & 0.2510 & 0 & 1 \\
\hline Full-time & 0.6698 & 0.4703 & 0 & 1 & 0.9236 & 0.2656 & 0 & 1 \\
\hline Works over $48 \mathrm{~h}$ & 0.3092 & 0.4622 & 0 & 1 & 0.6511 & 0.4766 & 0 & 1 \\
\hline Skill same as required & 0.4615 & 0.4985 & 0 & 1 & 0.3776 & 0.4848 & 0 & 1 \\
\hline Professional & 0.1135 & 0.3173 & 0 & 1 & 0.1330 & 0.3396 & 0 & 1 \\
\hline Associate prof. and technical & 0.1739 & 0.3790 & 0 & 1 & 0.1574 & 0.3642 & 0 & 1 \\
\hline Admin. and secretarial & 0.2795 & 0.4488 & 0 & 1 & 0.0864 & 0.2809 & 0 & 1 \\
\hline Skilled trades plant and mach. & 0.0361 & 0.1865 & 0 & 1 & 0.2657 & 0.4417 & 0 & 1 \\
\hline Personal and customer services & 0.2281 & 0.4197 & 0 & 1 & 0.0698 & 0.2548 & 0 & 1 \\
\hline Elementary occupations & 0.0878 & 0.2831 & 0 & 1 & 0.1244 & 0.3301 & 0 & 1 \\
\hline Gross weekly pay $\leq 110$ & 0.1463 & 0.3535 & 0 & 1 & 0.0324 & 0.1770 & 0 & 1 \\
\hline Gross weekly pay $111-180$ & 0.1511 & 0.3582 & 0 & 1 & 0.0359 & 0.1860 & 0 & 1 \\
\hline Gross weekly pay $181-260$ & 0.1961 & 0.3971 & 0 & 1 & 0.1256 & 0.3314 & 0 & 1 \\
\hline Gross weekly pay $261-360$ & 0.2052 & 0.4039 & 0 & 1 & 0.2091 & 0.4067 & 0 & 1 \\
\hline Trade union member & 0.3479 & 0.4763 & 0 & 1 & 0.3664 & 0.4818 & 0 & 1 \\
\hline Log workplace age & 3.2203 & 1.1470 & 0 & 6.802395 & 3.2816 & 1.1215 & 0 & 6.802395 \\
\hline Private establishment & 0.6205 & 0.4853 & 0 & 1 & 0.7824 & 0.4126 & 0 & 1 \\
\hline Sole establishment & 0.1985 & 0.3989 & 0 & 1 & 0.2208 & 0.4148 & 0 & 1 \\
\hline No. of employees/1000 & 0.3705 & 0.8382 & 0.005 & 7.74 & 0.3877 & 0.7366 & 0.005 & 7.74 \\
\hline Manufacturing & 0.0763 & 0.2655 & 0 & 1 & 0.2450 & 0.4301 & 0 & 1 \\
\hline Construction & 0.0237 & 0.1521 & 0 & 1 & 0.0820 & 0.2743 & 0 & 1 \\
\hline Wholesale and retail trade & 0.0986 & 0.2982 & 0 & 1 & 0.0863 & 0.2808 & 0 & 1 \\
\hline Hotel and restaurant & 0.0651 & 0.2467 & 0 & 1 & 0.1219 & 0.3272 & 0 & 1 \\
\hline Public and community services & 0.1513 & 0.3584 & 0 & 1 & 0.1644 & 0.3706 & 0 & 1 \\
\hline Education & 0.1724 & 0.3777 & 0 & 1 & 0.0634 & 0.2437 & 0 & 1 \\
\hline Health & 0.2341 & 0.4234 & 0 & 1 & 0.0601 & 0.2378 & 0 & 1 \\
\hline Urban area & 0.8487 & 0.3584 & 0 & 1 & 0.7951 & 0.4037 & 0 & 1 \\
\hline Unemployment to vacancy ratio & 3.4497 & 2.4207 & 0 & 10 & 3.3315 & 2.3770 & 0 & 10 \\
\hline Gender equality policy and practice & 0.1749 & 0.9800 & -1.2959 & 3.01558 & 0.0460 & 0.9944 & -1.2959 & 3.01558 \\
\hline No of observations & 9450 & & & & 8614 & & & \\
\hline
\end{tabular}

Table A4

Descriptive statistics on selected HRM policy and practice variables making up the EQUALITY variable $(\mathrm{N}=18,064)$.

\begin{tabular}{|c|c|c|c|c|}
\hline Variable & Mean & Std. dev. & Min & Max \\
\hline Workplace has formal equal opportunities (EO)/diversity policy & 0.8928 & 0.3094 & 0 & 1 \\
\hline Policy explicitly mention gender equality & 0.8232 & 0.3815 & 0 & 1 \\
\hline Training provided covered EO & 0.2544 & 0.4355 & 0 & 1 \\
\hline Meeting between senior mgt and workforce discuss EO & 0.2117 & 0.4086 & 0 & 1 \\
\hline Meeting between supervisors and workers discuss EO & 0.1948 & 0.3960 & 0 & 1 \\
\hline Meeting between mgt and employee committees discuss EO & 0.1113 & 0.3145 & 0 & 1 \\
\hline Employer monitors recruitment and screening to identify indirect discrimination by gender & 0.4204 & 0.4936 & 0 & 1 \\
\hline Employer reviews recruitment and screening to identify indirect discrimination by gender & 0.3279 & 0.4695 & 0 & 1 \\
\hline Employer monitors promotions to identify indirect discrimination by gender & 0.1976 & 0.3982 & 0 & 1 \\
\hline Employer reviews promotions to identify indirect discrimination by gender & 0.2060 & 0.4044 & 0 & 1 \\
\hline Employer reviews relative pay to identify indirect discrimination by gender & 0.1638 & 0.3701 & 0 & 1 \\
\hline Employer has special processes to encourage women in general & 0.0922 & 0.2893 & 0 & 1 \\
\hline
\end{tabular}


Table A5

Correlation matrix of HRM/EQUALITY variable and its constituent HRM policy and practice (HRMpp) dummies.

\begin{tabular}{|c|c|c|c|c|c|c|c|c|c|c|c|c|c|}
\hline & EQUALITY & HRMpp1 & HRMpp2 & HRMpp3 & HRMpp4 & HRMpp5 & HRMpp6 & HRMpp7 & HRMpp8 & HRMpp9 & HRMpp10 & HRMpp11 & HRMpp 12 \\
\hline EQUALITY & 1.0000 & & & & & & & & & & & & \\
\hline HRMpp1 & 0.4468 & 1.0000 & & & & & & & & & & & \\
\hline HRMpp2 & 0.4977 & 0.7478 & 1.0000 & & & & & & & & & & \\
\hline HRMpp3 & 0.4919 & 0.1642 & 0.1544 & 1.0000 & & & & & & & & & \\
\hline HRMpp4 & 0.2721 & 0.0990 & 0.0903 & 0.2396 & 1.0000 & & & & & & & & \\
\hline HRMpp5 & 0.3651 & 0.1393 & 0.1132 & 0.3030 & 0.3956 & 1.0000 & & & & & & & \\
\hline HRMpp6 & 0.3908 & 0.1050 & 0.1308 & 0.2392 & 0.0894 & 0.1887 & 1.0000 & & & & & & \\
\hline HRMpp7 & 0.7306 & 0.2593 & 0.3203 & 0.3039 & 0.0752 & 0.1487 & 0.2293 & 1.0000 & & & & & \\
\hline HRMpp8 & 0.7636 & 0.2108 & 0.2653 & 0.2478 & 0.0876 & 0.1192 & 0.2163 & 0.5952 & 1.0000 & & & & \\
\hline HRMpp9 & 0.7551 & 0.1581 & 0.2067 & 0.2620 & 0.1198 & 0.1709 & 0.1820 & 0.5019 & 0.5420 & 1.0000 & & & \\
\hline HRMpp10 & 0.7441 & 0.1575 & 0.2038 & 0.2331 & 0.0781 & 0.1318 & 0.1680 & 0.4431 & 0.6023 & 0.6575 & 1.0000 & & \\
\hline HRMpp11 & 0.5604 & 0.1200 & 0.1565 & 0.1269 & 0.0226 & 0.0933 & 0.1702 & 0.3317 & 0.3824 & 0.4175 & 0.4168 & 1.0000 & \\
\hline HRMpp12 & 0.2748 & 0.0486 & 0.0554 & 0.1606 & 0.0644 & 0.0603 & 0.1581 & 0.1249 & 0.1663 & 0.1413 & 0.1760 & 0.0845 & 1.0000 \\
\hline
\end{tabular}

Table 1

Workplace gender diversity and employee job satisfaction, main effect.

\begin{tabular}{|c|c|c|c|c|c|c|}
\hline & OLS & $\mathrm{RE}$ & OLS & RE & OLS & $\mathrm{RE}$ \\
\hline Gender diversity & $\begin{array}{l}-0.262^{* * *} \\
(0.044)\end{array}$ & $\begin{array}{l}-0.280^{\text {**** }} \\
(0.061)\end{array}$ & $\begin{array}{l}-0.108^{* *} \\
(0.046)\end{array}$ & $\begin{array}{l}-0.130^{* *} \\
(0.062)\end{array}$ & $\begin{array}{l}-0.095^{* *} \\
(0.047)\end{array}$ & $\begin{array}{l}-0.113^{*} \\
(0.062)\end{array}$ \\
\hline Majority female (female $>50 \%$ ) & $\begin{array}{l}0.084^{* * *} \\
(0.015)\end{array}$ & $\begin{array}{l}0.101^{* * * *} \\
(0.020)\end{array}$ & $\begin{array}{l}0.018 \\
(0.017)\end{array}$ & $\begin{array}{l}0.025 \\
(0.022)\end{array}$ & $\begin{array}{l}0.018 \\
(0.017)\end{array}$ & $\begin{array}{l}0.025 \\
(0.022)\end{array}$ \\
\hline Age $<30$ & $\begin{array}{l}-0.041^{* *} \\
(0.020)\end{array}$ & $\begin{array}{l}-0.040^{* *} \\
(0.019)\end{array}$ & $\begin{array}{c}-0.018 \\
(0.020)\end{array}$ & $\begin{array}{c}-0.029 \\
(0.019)\end{array}$ & $\begin{array}{l}-0.020 \\
(0.020)\end{array}$ & $\begin{array}{l}-0.030 \\
(0.019)\end{array}$ \\
\hline Age 30-39 & $\begin{array}{l}-0.004 \\
(0.018)\end{array}$ & $\begin{array}{l}-0.004 \\
(0.017)\end{array}$ & $\begin{array}{l}0.009 \\
(0.018)\end{array}$ & $\begin{array}{l}0.003 \\
(0.017)\end{array}$ & $\begin{array}{l}0.009 \\
(0.018)\end{array}$ & $\begin{array}{l}0.002 \\
(0.017)\end{array}$ \\
\hline Age $50+$ & $\begin{array}{l}0.116^{* * *} \\
(0.018)\end{array}$ & $\begin{array}{l}0.104^{* * *} \\
(0.017)\end{array}$ & $\begin{array}{l}0.100^{* * * *} \\
(0.018)\end{array}$ & $\begin{array}{l}0.097^{* * * *} \\
(0.017)\end{array}$ & $\begin{array}{l}0.100^{* * * *} \\
(0.018)\end{array}$ & $\begin{array}{l}0.096^{* * * *} \\
(0.017)\end{array}$ \\
\hline Female & $\begin{array}{l}0.097^{* * * *} \\
(0.016)\end{array}$ & $\begin{array}{l}0.078^{* * *} \\
(0.016)\end{array}$ & $\begin{array}{l}0.078^{* * * *} \\
(0.016)\end{array}$ & $\begin{array}{l}0.070^{* * *} \\
(0.016)\end{array}$ & $\begin{array}{l}0.078^{* * *} \\
(0.016)\end{array}$ & $\begin{array}{l}0.070^{* * *} \\
(0.016)\end{array}$ \\
\hline Married & $\begin{array}{l}0.059 * * * \\
(0.015)\end{array}$ & $\begin{array}{l}0.052^{* * *} \\
(0.014)\end{array}$ & $\begin{array}{l}0.059 * * * \\
(0.014)\end{array}$ & $\begin{array}{l}0.053^{* * *} \\
(0.014)\end{array}$ & $\begin{array}{l}0.059^{* * *} \\
(0.014)\end{array}$ & $\begin{array}{l}0.053^{* * *} \\
(0.014)\end{array}$ \\
\hline White & $\begin{array}{l}-0.022 \\
(0.029)\end{array}$ & $\begin{array}{l}-0.034 \\
(0.029)\end{array}$ & $\begin{array}{l}-0.035 \\
(0.029)\end{array}$ & $\begin{array}{l}-0.043 \\
(0.029)\end{array}$ & $\begin{array}{l}-0.036 \\
(0.029)\end{array}$ & $\begin{array}{l}-0.044 \\
(0.029)\end{array}$ \\
\hline Children $<7$ years old & $\begin{array}{l}0.014 \\
(0.018)\end{array}$ & $\begin{array}{l}0.020 \\
(0.018)\end{array}$ & $\begin{array}{l}0.013 \\
(0.018)\end{array}$ & $\begin{array}{l}0.019 \\
(0.018)\end{array}$ & $\begin{array}{l}0.013 \\
(0.018)\end{array}$ & $\begin{array}{l}0.019 \\
(0.018)\end{array}$ \\
\hline Other dependents & $\begin{array}{l}-0.061^{* * *} \\
(0.018)\end{array}$ & $\begin{array}{l}-0.064^{* * *} \\
(0.017)\end{array}$ & $\begin{array}{l}-0.066^{* * *} \\
(0.018)\end{array}$ & $\begin{array}{l}-0.067^{* * *} \\
(0.017)\end{array}$ & $\begin{array}{l}-0.066^{* * *} \\
(0.018)\end{array}$ & $\begin{array}{l}-0.066^{* * *} \\
(0.017)\end{array}$ \\
\hline Disabled & $\begin{array}{l}-0.123^{* * * *} \\
(0.021)\end{array}$ & $\begin{array}{l}-0.112^{* * *} \\
(0.019)\end{array}$ & $\begin{array}{l}-0.117^{* * *} \\
(0.021)\end{array}$ & $\begin{array}{l}-0.110^{* * *} \\
(0.019)\end{array}$ & $\begin{array}{l}-0.116^{* * *} \\
(0.021)\end{array}$ & $\begin{array}{l}-0.109^{\text {**** }} \\
(0.019)\end{array}$ \\
\hline No academic qualification & $\begin{array}{l}0.147^{* * *} \\
(0.026)\end{array}$ & $\begin{array}{l}0.147^{* * *} \\
(0.025)\end{array}$ & $\begin{array}{l}0.160^{* * * *} \\
(0.026)\end{array}$ & $\begin{array}{l}0.154^{* * *} \\
(0.025)\end{array}$ & $\begin{array}{l}0.158^{* * *} \\
(0.026)\end{array}$ & $\begin{array}{l}0.153^{* * *} \\
(0.025)\end{array}$ \\
\hline O-level & $\begin{array}{l}0.076^{* * * *} \\
(0.021)\end{array}$ & $\begin{array}{l}0.086^{* * *} \\
(0.021)\end{array}$ & $\begin{array}{l}0.089^{* * *} \\
(0.021)\end{array}$ & $\begin{array}{l}0.092^{* * *} \\
(0.021)\end{array}$ & $\begin{array}{l}0.088^{* * *} \\
(0.021)\end{array}$ & $\begin{array}{l}0.091^{\text {**** }} \\
(0.021)\end{array}$ \\
\hline A-level & $\begin{array}{l}-0.006 \\
(0.026)\end{array}$ & $\begin{array}{l}0.022 \\
(0.025)\end{array}$ & $\begin{array}{l}0.015 \\
(0.026)\end{array}$ & $\begin{array}{l}0.031 \\
(0.025)\end{array}$ & $\begin{array}{l}0.014 \\
(0.026)\end{array}$ & $\begin{array}{l}0.031 \\
(0.025)\end{array}$ \\
\hline Other qualification & $\begin{array}{l}0.062^{* * *} \\
(0.018)\end{array}$ & $\begin{array}{l}0.062^{* * *} \\
(0.018)\end{array}$ & $\begin{array}{l}0.061^{\text {**** }} \\
(0.018)\end{array}$ & $\begin{array}{l}0.061^{\text {**** }} \\
(0.018)\end{array}$ & $\begin{array}{l}0.060^{\text {**** }} \\
(0.018)\end{array}$ & $\begin{array}{l}0.061^{* * *} \\
(0.018)\end{array}$ \\
\hline On permanent contract & $\begin{array}{l}0.012 \\
(0.024)\end{array}$ & $\begin{array}{l}0.036 \\
(0.024)\end{array}$ & $\begin{array}{l}0.041^{*} \\
(0.024)\end{array}$ & $\begin{array}{l}0.050^{* *} \\
(0.024)\end{array}$ & $\begin{array}{l}0.040^{*} \\
(0.024)\end{array}$ & $\begin{array}{l}0.049^{* *} \\
(0.024)\end{array}$ \\
\hline Full-time & $\begin{array}{l}-0.033 \\
(0.022)\end{array}$ & $\begin{array}{l}-0.035 \\
(0.022)\end{array}$ & $\begin{array}{l}-0.032 \\
(0.022)\end{array}$ & $\begin{array}{l}-0.035 \\
(0.022)\end{array}$ & $\begin{array}{l}-0.032 \\
(0.022)\end{array}$ & $\begin{array}{l}-0.035 \\
(0.022)\end{array}$ \\
\hline Works over $48 \mathrm{~h}$ & $\begin{array}{l}0.059^{* * * *} \\
(0.015)\end{array}$ & $\begin{array}{l}0.051^{* * * *} \\
(0.015)\end{array}$ & $\begin{array}{l}0.044^{* * * *} \\
(0.015)\end{array}$ & $\begin{array}{l}0.044^{* * *} \\
(0.015)\end{array}$ & $\begin{array}{l}0.043^{* * *} \\
(0.015)\end{array}$ & $\begin{array}{l}0.043^{* * *} \\
(0.015)\end{array}$ \\
\hline Skill same as required & $\begin{array}{l}0.280^{* * * *} \\
(0.012)\end{array}$ & $\begin{array}{l}0.261^{* * *} \\
(0.012)\end{array}$ & $\begin{array}{l}0.272^{* * *} \\
(0.012)\end{array}$ & $\begin{array}{l}0.258^{* * *} \\
(0.012)\end{array}$ & $\begin{array}{l}0.272^{* * * *} \\
(0.012)\end{array}$ & $\begin{array}{l}0.258^{* * *} \\
(0.012)\end{array}$ \\
\hline Professional & $\begin{array}{l}-0.200^{\text {**** }} \\
(0.025)\end{array}$ & $\begin{array}{l}-0.234^{* * *} \\
(0.027)\end{array}$ & $\begin{array}{l}-0.245^{* * * *} \\
(0.026)\end{array}$ & $\begin{array}{l}-0.256^{* * * *} \\
(0.027)\end{array}$ & $\begin{array}{l}-0.246^{* * * *} \\
(0.026)\end{array}$ & $\begin{array}{l}-0.256^{* * *} \\
(0.027)\end{array}$ \\
\hline Associate prof. and technical & $\begin{array}{l}-0.205^{* * *} \\
(0.024)\end{array}$ & $\begin{array}{l}-0.225^{* * *} \\
(0.024)\end{array}$ & $\begin{array}{l}-0.220^{* * * *} \\
(0.024)\end{array}$ & $\begin{array}{l}-0.230^{* * * *} \\
(0.024)\end{array}$ & $\begin{array}{l}-0.220^{* * *} \\
(0.024)\end{array}$ & $\begin{array}{l}-0.230^{\text {**** }} \\
(0.024)\end{array}$ \\
\hline Admin. and secretarial & $\begin{array}{l}-0.358^{* * *} \\
(0.025)\end{array}$ & $\begin{array}{l}-0.331^{* * *} \\
(0.025)\end{array}$ & $\begin{array}{l}-0.341^{* * *} \\
(0.025)\end{array}$ & $\begin{array}{l}-0.322^{\text {**** }} \\
(0.025)\end{array}$ & $\begin{array}{l}-0.341^{\text {**** }} \\
(0.025)\end{array}$ & $\begin{array}{l}-0.321^{* * *} \\
(0.025)\end{array}$ \\
\hline Skilled trades plant and mach. & $\begin{array}{l}-0.404^{* * *} \\
(0.027)\end{array}$ & $\begin{array}{l}-0.383^{* * *} \\
(0.027)\end{array}$ & $\begin{array}{l}-0.387^{* * *} \\
(0.027)\end{array}$ & $\begin{array}{l}-0.375^{* * *} \\
(0.027)\end{array}$ & $\begin{array}{l}-0.387^{* * *} \\
(0.027)\end{array}$ & $\begin{array}{l}-0.375^{* * *} \\
(0.027)\end{array}$ \\
\hline Personal and customer services & $\begin{array}{l}-0.295^{\text {**** }} \\
(0.027)\end{array}$ & $\begin{array}{l}-0.281^{\text {**** }} \\
(0.028)\end{array}$ & $\begin{array}{l}-0.323^{* * *} \\
(0.027)\end{array}$ & $\begin{array}{l}-0.299^{* * * *} \\
(0.028)\end{array}$ & $\begin{array}{l}-0.323^{\text {**** }} \\
(0.027)\end{array}$ & $\begin{array}{l}-0.299^{* * *} \\
(0.028)\end{array}$ \\
\hline Elementary occupations & $\begin{array}{l}-0.364^{* * *} \\
(0.030)\end{array}$ & $\begin{array}{l}-0.349^{* * *} \\
(0.030)\end{array}$ & $\begin{array}{l}-0.347^{* * * *} \\
(0.030)\end{array}$ & $\begin{array}{l}-0.337^{* * * *} \\
(0.030)\end{array}$ & $\begin{array}{l}-0.348^{* * *} \\
(0.030)\end{array}$ & $\begin{array}{l}-0.337^{\text {*** }} \\
(0.030)\end{array}$ \\
\hline Gross weekly pay $\leq 110$ & $\begin{array}{l}-0.033 \\
(0.034)\end{array}$ & $\begin{array}{l}-0.105^{* * *} \\
(0.035)\end{array}$ & $\begin{array}{l}-0.076^{* *} \\
(0.034)\end{array}$ & $\begin{array}{l}-0.130^{* * *} \\
(0.035)\end{array}$ & $\begin{array}{l}-0.079^{* *} \\
(0.034)\end{array}$ & $\begin{array}{l}-0.133^{* * *} \\
(0.035)\end{array}$ \\
\hline Gross weekly pay $111-180$ & $\begin{array}{l}-0.092^{\text {**** }} \\
(0.030)\end{array}$ & $\begin{array}{l}-0.146^{* * *} \\
(0.029)\end{array}$ & $\begin{array}{l}-0.136^{* * * *} \\
(0.030)\end{array}$ & $\begin{array}{l}-0.170^{* * * *} \\
(0.029)\end{array}$ & $\begin{array}{l}-0.138^{* * *} \\
(0.030)\end{array}$ & $\begin{array}{l}-0.172^{* * *} \\
(0.029)\end{array}$ \\
\hline Gross weekly pay $181-260$ & $\begin{array}{l}-0.120^{\text {**** }} \\
(0.022)\end{array}$ & $\begin{array}{l}-0.149^{\text {*** }} \\
(0.022)\end{array}$ & $\begin{array}{l}-0.148^{* * *} \\
(0.022)\end{array}$ & $\begin{array}{l}-0.164^{* * *} \\
(0.022)\end{array}$ & $\begin{array}{l}-0.150^{\text {**** }} \\
(0.022)\end{array}$ & $\begin{array}{l}-0.166^{* * *} \\
(0.022)\end{array}$ \\
\hline
\end{tabular}


Table 1 (continued)

\begin{tabular}{|c|c|c|c|c|c|c|}
\hline & OLS & $\mathrm{RE}$ & OLS & $\mathrm{RE}$ & OLS & $\mathrm{RE}$ \\
\hline Gross weekly pay $261-360$ & $\begin{array}{l}-0.139^{* * *} \\
(0.019)\end{array}$ & $\begin{array}{l}-0.148^{* * *} \\
(0.018)\end{array}$ & $\begin{array}{l}-0.154^{* * *} \\
(0.018)\end{array}$ & $\begin{array}{l}-0.156^{* * *} \\
(0.018)\end{array}$ & $\begin{array}{l}-0.155^{* * *} \\
(0.018)\end{array}$ & $\begin{array}{l}-0.157^{* * *} \\
(0.018)\end{array}$ \\
\hline Trade union member & $\begin{array}{l}-0.203^{* * * *} \\
(0.014)\end{array}$ & $\begin{array}{l}-0.174^{* * * *} \\
(0.015)\end{array}$ & $\begin{array}{l}-0.179^{* * *} \\
(0.015)\end{array}$ & $\begin{array}{l}-0.157^{* * *} \\
(0.016)\end{array}$ & $\begin{array}{l}-0.177^{* * *} \\
(0.015)\end{array}$ & $\begin{array}{l}-0.155^{\text {*** }} \\
(0.016)\end{array}$ \\
\hline Log workplace age & & & $\begin{array}{l}-0.019^{* * *} \\
(0.006)\end{array}$ & $\begin{array}{l}-0.019^{* *} \\
(0.008)\end{array}$ & $\begin{array}{l}-0.019^{* * *} \\
(0.006)\end{array}$ & $\begin{array}{l}-0.019^{* *} \\
(0.008)\end{array}$ \\
\hline Private establishment & & & $\begin{array}{l}0.068^{* * *} \\
(0.019)\end{array}$ & $\begin{array}{l}0.080^{* * * *} \\
(0.026)\end{array}$ & $\begin{array}{l}0.062^{* * *} \\
(0.019)\end{array}$ & $\begin{array}{l}0.072^{* * * *} \\
(0.026)\end{array}$ \\
\hline Sole establishment & & & $\begin{array}{l}0.123^{* * *} \\
(0.016)\end{array}$ & $\begin{array}{l}0.141^{* * *} \\
(0.022)\end{array}$ & $\begin{array}{l}0.119^{* * *} \\
(0.016)\end{array}$ & $\begin{array}{l}0.134^{* * *} \\
(0.022)\end{array}$ \\
\hline No. of employees/1000 & & & $\begin{array}{l}-0.050^{* * *} \\
(0.008)\end{array}$ & $\begin{array}{l}-0.054^{* * *} \\
(0.012)\end{array}$ & $\begin{array}{l}-0.044^{* * *} \\
(0.009)\end{array}$ & $\begin{array}{l}-0.046^{\text {**** }} \\
(0.012)\end{array}$ \\
\hline Manufacturing & & & $\begin{array}{l}-0.041^{*} \\
(0.025)\end{array}$ & $\begin{array}{l}-0.047 \\
(0.034)\end{array}$ & $\begin{array}{l}-0.045^{*} \\
(0.025)\end{array}$ & $\begin{array}{l}-0.052 \\
(0.034)\end{array}$ \\
\hline Construction & & & $\begin{array}{l}0.166^{* * * *} \\
(0.032)\end{array}$ & $\begin{array}{l}0.156^{* * *} \\
(0.046)\end{array}$ & $\begin{array}{l}0.165^{* * *} \\
(0.032)\end{array}$ & $\begin{array}{l}0.155^{* * *} \\
(0.046)\end{array}$ \\
\hline Wholesale and retail trade & & & $\begin{array}{l}0.093^{* * *} \\
(0.026)\end{array}$ & $\begin{array}{l}0.102^{* * *} \\
(0.035)\end{array}$ & $\begin{array}{l}0.090^{* * * *} \\
(0.026)\end{array}$ & $\begin{array}{l}0.098^{* * *} \\
(0.035)\end{array}$ \\
\hline Hotel and restaurant & & & $\begin{array}{l}0.025 \\
(0.028)\end{array}$ & $\begin{array}{l}0.018 \\
(0.036)\end{array}$ & $\begin{array}{l}0.023 \\
(0.028)\end{array}$ & $\begin{array}{l}0.015 \\
(0.036)\end{array}$ \\
\hline Public and community services & & & $\begin{array}{l}0.105^{* * *} \\
(0.024)\end{array}$ & $\begin{array}{l}0.114^{* * * *} \\
(0.033)\end{array}$ & $\begin{array}{l}0.113^{* * * *} \\
(0.025)\end{array}$ & $\begin{array}{l}0.124^{* * *} \\
(0.034)\end{array}$ \\
\hline Education & & & $\begin{array}{l}0.292^{* * *} \\
(0.029)\end{array}$ & $\begin{array}{l}0.297^{* * *} \\
(0.040)\end{array}$ & $\begin{array}{l}0.296^{* * *} \\
(0.029)\end{array}$ & $\begin{array}{l}0.303^{* * *} \\
(0.040)\end{array}$ \\
\hline Health & & & $\begin{array}{l}0.289^{* * *} \\
(0.026)\end{array}$ & $\begin{array}{l}0.296^{* * * *} \\
(0.036)\end{array}$ & $\begin{array}{l}0.292^{* * *} \\
(0.026)\end{array}$ & $\begin{array}{l}0.300^{* * *} \\
(0.036)\end{array}$ \\
\hline Urban area & & & $\begin{array}{l}-0.055^{* * *} \\
(0.016)\end{array}$ & $\begin{array}{l}-0.057^{* *} \\
(0.023)\end{array}$ & $\begin{array}{l}-0.053^{* * * *} \\
(0.016)\end{array}$ & $\begin{array}{l}-0.054^{* *} \\
(0.023)\end{array}$ \\
\hline Unemployment to vacancy ratio & & & $\begin{array}{l}-0.006^{* *} \\
(0.003)\end{array}$ & $\begin{array}{l}-0.008^{* *} \\
(0.004)\end{array}$ & $\begin{array}{l}-0.005^{* *} \\
(0.003)\end{array}$ & $\begin{array}{l}-0.007^{*} \\
(0.004)\end{array}$ \\
\hline Gender equality policy and practice & & & & & $\begin{array}{l}-0.016^{* *} \\
(0.008)\end{array}$ & $\begin{array}{l}-0.022^{* *} \\
(0.011)\end{array}$ \\
\hline Constant & $\begin{array}{l}4.537^{* * * *} \\
(0.053)\end{array}$ & $\begin{array}{l}4.560^{* * * *} \\
(0.055)\end{array}$ & $\begin{array}{l}4.492^{* * *} \\
(0.064)\end{array}$ & $\begin{array}{l}4.513^{* * *} \\
(0.073)\end{array}$ & $\begin{array}{l}4.492^{* * *} \\
(0.064)\end{array}$ & $\begin{array}{l}4.511^{* * *} \\
(0.073)\end{array}$ \\
\hline $\begin{array}{l}\text { No. of employees } \\
\text { R-squared }\end{array}$ & $\begin{array}{l}18,064 \\
0.091\end{array}$ & 18,064 & $\begin{array}{l}18,064 \\
0.109\end{array}$ & 18,064 & $\begin{array}{l}18,064 \\
0.110\end{array}$ & 18,064 \\
\hline No. of workplaces & & 1506 & & 1506 & & 1506 \\
\hline
\end{tabular}

Robust standard errors in parentheses.

${ }^{* * *} \mathrm{p}<0.01,{ }^{* *} \mathrm{p}<0.05$, and ${ }^{*} \mathrm{p}<0.1$.

Table 2

Workplace gender diversity and employee job satisfaction, sub-group analysis (female).

\begin{tabular}{|c|c|c|c|c|c|c|}
\hline & OLS & RE & OLS & $\mathrm{RE}$ & OLS & RE \\
\hline Gender diversity & $\begin{array}{l}-0.496^{* * *} \\
(0.057)\end{array}$ & $\begin{array}{l}-0.493^{* * *} \\
(0.077)\end{array}$ & $\begin{array}{l}-0.287^{* * *} \\
(0.062)\end{array}$ & $\begin{array}{l}-0.295^{* * *} \\
(0.079)\end{array}$ & $\begin{array}{l}-0.263^{* * *} \\
(0.063)\end{array}$ & $\begin{array}{l}-0.273^{* * *} \\
(0.080)\end{array}$ \\
\hline Majority female (female > 50\%) & $\begin{array}{l}0.049^{* *} \\
(0.021)\end{array}$ & $\begin{array}{l}0.067^{* * *} \\
(0.026)\end{array}$ & $\begin{array}{l}0.008 \\
(0.023)\end{array}$ & $\begin{array}{l}0.015 \\
(0.028)\end{array}$ & $\begin{array}{l}0.007 \\
(0.023)\end{array}$ & $\begin{array}{l}0.015 \\
(0.028)\end{array}$ \\
\hline Age $<30$ & $\begin{array}{l}-0.072^{\text {**** }} \\
(0.026)\end{array}$ & $\begin{array}{l}-0.063^{* *} \\
(0.025)\end{array}$ & $\begin{array}{l}-0.048^{*} \\
(0.026)\end{array}$ & $\begin{array}{l}-0.049^{* *} \\
(0.025)\end{array}$ & $\begin{array}{l}-0.050^{*} \\
(0.026)\end{array}$ & $\begin{array}{l}-0.050^{* *} \\
(0.025)\end{array}$ \\
\hline Age 30-39 & $\begin{array}{l}0.024 \\
(0.024)\end{array}$ & $\begin{array}{l}0.023 \\
(0.023)\end{array}$ & $\begin{array}{l}0.039 \\
(0.024)\end{array}$ & $\begin{array}{l}0.032 \\
(0.023)\end{array}$ & $\begin{array}{l}0.038 \\
(0.024)\end{array}$ & $\begin{array}{l}0.032 \\
(0.023)\end{array}$ \\
\hline Age $50+$ & $\begin{array}{l}0.083^{* * *} \\
(0.024)\end{array}$ & $\begin{array}{l}0.072^{* * *} \\
(0.023)\end{array}$ & $\begin{array}{l}0.066^{* * *} \\
(0.023)\end{array}$ & $\begin{array}{l}0.063^{* * *} \\
(0.023)\end{array}$ & $\begin{array}{l}0.065^{* * *} \\
(0.023)\end{array}$ & $\begin{array}{l}0.063^{* * *} \\
(0.023)\end{array}$ \\
\hline Married & $\begin{array}{l}0.044^{* *} \\
(0.019)\end{array}$ & $\begin{array}{l}0.036^{* *} \\
(0.018)\end{array}$ & $\begin{array}{l}0.041^{* *} \\
(0.019)\end{array}$ & $\begin{array}{l}0.035^{*} \\
(0.018)\end{array}$ & $\begin{array}{l}0.040^{* *} \\
(0.019)\end{array}$ & $\begin{array}{l}0.035^{*} \\
(0.018)\end{array}$ \\
\hline White & $\begin{array}{l}0.020 \\
(0.039)\end{array}$ & $\begin{array}{l}0.008 \\
(0.038)\end{array}$ & $\begin{array}{l}0.007 \\
(0.039)\end{array}$ & $\begin{array}{l}-0.006 \\
(0.039)\end{array}$ & $\begin{array}{l}0.004 \\
(0.039)\end{array}$ & $\begin{array}{l}-0.007 \\
(0.039)\end{array}$ \\
\hline Children $<7$ years old & $\begin{array}{l}-0.010 \\
(0.026)\end{array}$ & $\begin{array}{l}0.004 \\
(0.025)\end{array}$ & $\begin{array}{l}-0.010 \\
(0.026)\end{array}$ & $\begin{array}{l}0.002 \\
(0.025)\end{array}$ & $\begin{array}{l}-0.008 \\
(0.026)\end{array}$ & $\begin{array}{l}0.004 \\
(0.025)\end{array}$ \\
\hline Other dependents & $\begin{array}{l}-0.057^{* *} \\
(0.023)\end{array}$ & $\begin{array}{l}-0.055^{* * * *} \\
(0.021)\end{array}$ & $\begin{array}{l}-0.062^{* * *} \\
(0.022)\end{array}$ & $\begin{array}{l}-0.059^{* * *} \\
(0.021)\end{array}$ & $\begin{array}{l}-0.060^{* * *} \\
(0.022)\end{array}$ & $\begin{array}{l}-0.058^{* * *} \\
(0.021)\end{array}$ \\
\hline
\end{tabular}


Table 2 (continued)

\begin{tabular}{|c|c|c|c|c|c|c|}
\hline & OLS & $\mathrm{RE}$ & OLS & $\mathrm{RE}$ & OLS & $\mathrm{RE}$ \\
\hline Disabled & $\begin{array}{l}-0.105^{* * * *} \\
(0.029)\end{array}$ & $\begin{array}{l}-0.089^{* * * *} \\
(0.026)\end{array}$ & $\begin{array}{l}-0.096^{* * *} \\
(0.028)\end{array}$ & $\begin{array}{l}-0.085^{* * * *} \\
(0.026)\end{array}$ & $\begin{array}{l}-0.095^{* * * *} \\
(0.028)\end{array}$ & $\begin{array}{l}-0.084^{* * *} \\
(0.026)\end{array}$ \\
\hline No academic qualification & $\begin{array}{l}0.114^{* * * *} \\
(0.036)\end{array}$ & $\begin{array}{l}0.119^{* * * *} \\
(0.036)\end{array}$ & $\begin{array}{l}0.139^{* * * *} \\
(0.036)\end{array}$ & $\begin{array}{l}0.135^{* * * *} \\
(0.036)\end{array}$ & $\begin{array}{l}0.137^{* * * *} \\
(0.036)\end{array}$ & $\begin{array}{l}0.134^{* * *} \\
(0.036)\end{array}$ \\
\hline O-level & $\begin{array}{l}0.090^{* * *} \\
(0.029)\end{array}$ & $\begin{array}{l}0.101^{* * * *} \\
(0.028)\end{array}$ & $\begin{array}{l}0.111^{* * * *} \\
(0.028)\end{array}$ & $\begin{array}{l}0.113^{* * * *} \\
(0.028)\end{array}$ & $\begin{array}{l}0.110^{* * * *} \\
(0.028)\end{array}$ & $\begin{array}{l}0.112^{* * *} \\
(0.028)\end{array}$ \\
\hline A-level & $\begin{array}{c}-0.032 \\
(0.035)\end{array}$ & $\begin{array}{l}0.000 \\
(0.034)\end{array}$ & $\begin{array}{l}-0.010 \\
(0.035)\end{array}$ & $\begin{array}{l}0.010 \\
(0.034)\end{array}$ & $\begin{array}{c}-0.011 \\
(0.035)\end{array}$ & $\begin{array}{l}0.009 \\
(0.034)\end{array}$ \\
\hline Other qualification & $\begin{array}{l}0.075^{* * *} \\
(0.025)\end{array}$ & $\begin{array}{l}0.075^{* * * *} \\
(0.025)\end{array}$ & $\begin{array}{l}0.077^{* * * *} \\
(0.025)\end{array}$ & $\begin{array}{l}0.076^{* * * *} \\
(0.025)\end{array}$ & $\begin{array}{l}0.076^{* * *} \\
(0.025)\end{array}$ & $\begin{array}{l}0.075^{* * *} \\
(0.025)\end{array}$ \\
\hline On permanent contract & $\begin{array}{l}0.018 \\
(0.029)\end{array}$ & $\begin{array}{l}0.036 \\
(0.030)\end{array}$ & $\begin{array}{l}0.043 \\
(0.029)\end{array}$ & $\begin{array}{l}0.050^{*} \\
(0.030)\end{array}$ & $\begin{array}{l}0.041 \\
(0.029)\end{array}$ & $\begin{array}{l}0.049 \\
(0.030)\end{array}$ \\
\hline Full-time & $\begin{array}{l}-0.005 \\
(0.026)\end{array}$ & $\begin{array}{l}-0.010 \\
(0.025)\end{array}$ & $\begin{array}{l}-0.014 \\
(0.026)\end{array}$ & $\begin{array}{l}-0.016 \\
(0.025)\end{array}$ & $\begin{array}{l}-0.016 \\
(0.026)\end{array}$ & $\begin{array}{l}-0.016 \\
(0.025)\end{array}$ \\
\hline Works over $48 \mathrm{~h}$ & $\begin{array}{l}0.054^{* * *} \\
(0.021)\end{array}$ & $\begin{array}{l}0.034^{*} \\
(0.020)\end{array}$ & $\begin{array}{l}0.035^{*} \\
(0.021)\end{array}$ & $\begin{array}{l}0.024 \\
(0.020)\end{array}$ & $\begin{array}{l}0.035^{*} \\
(0.021)\end{array}$ & $\begin{array}{l}0.024 \\
(0.020)\end{array}$ \\
\hline Skill same as required & $\begin{array}{l}0.288^{* * * *} \\
(0.016)\end{array}$ & $\begin{array}{l}0.271^{* * * *} \\
(0.016)\end{array}$ & $\begin{array}{l}0.286^{* * * *} \\
(0.016)\end{array}$ & $\begin{array}{l}0.271^{* * * *} \\
(0.016)\end{array}$ & $\begin{array}{l}0.285^{* * * *} \\
(0.016)\end{array}$ & $\begin{array}{l}0.271^{* * *} \\
(0.016)\end{array}$ \\
\hline Professional & $\begin{array}{l}-0.145^{* * *} \\
(0.038)\end{array}$ & $\begin{array}{l}-0.171^{* * *} \\
(0.040)\end{array}$ & $\begin{array}{l}-0.182^{* * *} \\
(0.039)\end{array}$ & $\begin{array}{l}-0.193^{* * *} \\
(0.041)\end{array}$ & $\begin{array}{l}-0.182^{* * *} \\
(0.039)\end{array}$ & $\begin{array}{l}-0.193^{* * *} \\
(0.041)\end{array}$ \\
\hline Associate prof. and technical & $\begin{array}{l}-0.172^{* * *} \\
(0.035)\end{array}$ & $\begin{array}{l}-0.194^{* * *} \\
(0.036)\end{array}$ & $\begin{array}{l}-0.184^{* * *} \\
(0.035)\end{array}$ & $\begin{array}{l}-0.197^{* * *} \\
(0.036)\end{array}$ & $\begin{array}{l}-0.184^{* * *} \\
(0.035)\end{array}$ & $\begin{array}{l}-0.197^{* * *} \\
(0.036)\end{array}$ \\
\hline Admin. and secretarial & $\begin{array}{l}-0.330^{* * *} \\
(0.035)\end{array}$ & $\begin{array}{l}-0.304^{* * *} \\
(0.035)\end{array}$ & $\begin{array}{l}-0.312^{* * *} \\
(0.035)\end{array}$ & $\begin{array}{l}-0.295^{* * *} \\
(0.035)\end{array}$ & $\begin{array}{l}-0.312^{* * *} \\
(0.035)\end{array}$ & $\begin{array}{l}-0.295^{* * *} \\
(0.035)\end{array}$ \\
\hline Skilled trades plant and mach. & $\begin{array}{l}-0.453^{* * * *} \\
(0.057)\end{array}$ & $\begin{array}{l}-0.418^{* * * *} \\
(0.057)\end{array}$ & $\begin{array}{l}-0.412^{* * * *} \\
(0.059)\end{array}$ & $\begin{array}{l}-0.390^{* * * *} \\
(0.058)\end{array}$ & $\begin{array}{l}-0.413^{* * * *} \\
(0.059)\end{array}$ & $\begin{array}{l}-0.391^{* * *} \\
(0.058)\end{array}$ \\
\hline Personal and customer services & $\begin{array}{l}-0.243^{* * *} \\
(0.037)\end{array}$ & $\begin{array}{l}-0.233^{* * *} \\
(0.038)\end{array}$ & $\begin{array}{l}-0.260^{* * *} \\
(0.038)\end{array}$ & $\begin{array}{l}-0.245^{* * *} \\
(0.038)\end{array}$ & $\begin{array}{l}-0.260^{* * *} \\
(0.038)\end{array}$ & $\begin{array}{l}-0.245^{* * *} \\
(0.038)\end{array}$ \\
\hline Elementary occupations & $\begin{array}{l}-0.273^{* * *} \\
(0.044)\end{array}$ & $\begin{array}{l}-0.271^{* * *} \\
(0.045)\end{array}$ & $\begin{array}{l}-0.279^{* * *} \\
(0.045)\end{array}$ & $\begin{array}{l}-0.274^{* * *} \\
(0.046)\end{array}$ & $\begin{array}{l}-0.280^{* * * *} \\
(0.045)\end{array}$ & $\begin{array}{l}-0.274^{* * *} \\
(0.046)\end{array}$ \\
\hline Gross weekly pay $\leq 110$ & $\begin{array}{l}-0.052 \\
(0.041)\end{array}$ & $\begin{array}{l}-0.121^{* * * *} \\
(0.041)\end{array}$ & $\begin{array}{l}-0.091^{* *} \\
(0.041)\end{array}$ & $\begin{array}{l}-0.147^{* * *} \\
(0.041)\end{array}$ & $\begin{array}{l}-0.097^{* *} \\
(0.041)\end{array}$ & $\begin{array}{l}-0.151^{* * *} \\
(0.041)\end{array}$ \\
\hline Gross weekly pay $111-180$ & $\begin{array}{l}-0.103^{* * *} \\
(0.036)\end{array}$ & $\begin{array}{l}-0.152^{* * *} \\
(0.035)\end{array}$ & $\begin{array}{l}-0.154^{* * *} \\
(0.036)\end{array}$ & $\begin{array}{l}-0.183^{* * *} \\
(0.035)\end{array}$ & $\begin{array}{l}-0.158^{* * *} \\
(0.036)\end{array}$ & $\begin{array}{l}-0.186^{* * *} \\
(0.035)\end{array}$ \\
\hline Gross weekly pay $181-260$ & $\begin{array}{l}-0.099^{* * * *} \\
(0.029)\end{array}$ & $\begin{array}{l}-0.128^{* * *} \\
(0.028)\end{array}$ & $\begin{array}{l}-0.136^{* * *} \\
(0.029)\end{array}$ & $\begin{array}{l}-0.150^{* * *} \\
(0.028)\end{array}$ & $\begin{array}{l}-0.139^{* * *} \\
(0.029)\end{array}$ & $\begin{array}{l}-0.153^{* * *} \\
(0.028)\end{array}$ \\
\hline Gross weekly pay $261-360$ & $\begin{array}{l}-0.095^{* * *} \\
(0.026)\end{array}$ & $\begin{array}{l}-0.113^{* * *} \\
(0.026)\end{array}$ & $\begin{array}{l}-0.118^{* * *} \\
(0.026)\end{array}$ & $\begin{array}{l}-0.127^{* * *} \\
(0.026)\end{array}$ & $\begin{array}{l}-0.120^{* * *} \\
(0.026)\end{array}$ & $\begin{array}{l}-0.128^{* * *} \\
(0.026)\end{array}$ \\
\hline Trade union member & $\begin{array}{l}-0.163^{* * *} \\
(0.019)\end{array}$ & $\begin{array}{l}-0.139^{* * *} \\
(0.020)\end{array}$ & $\begin{array}{l}-0.133^{* * *} \\
(0.020)\end{array}$ & $\begin{array}{l}-0.124^{* * *} \\
(0.021)\end{array}$ & $\begin{array}{l}-0.131^{* * *} \\
(0.020)\end{array}$ & $\begin{array}{l}-0.123^{* * *} \\
(0.021)\end{array}$ \\
\hline Log workplace age & & & $\begin{array}{l}-0.021^{* * *} \\
(0.008)\end{array}$ & $\begin{array}{l}-0.022^{* *} \\
(0.010)\end{array}$ & $\begin{array}{l}-0.021^{* * *} \\
(0.008)\end{array}$ & $\begin{array}{l}-0.022^{* *} \\
(0.010)\end{array}$ \\
\hline Private establishment & & & $\begin{array}{l}0.120^{* * * *} \\
(0.025)\end{array}$ & $\begin{array}{l}0.111^{* * *} \\
(0.032)\end{array}$ & $\begin{array}{l}0.113^{* * *} \\
(0.025)\end{array}$ & $\begin{array}{l}0.102^{* * *} \\
(0.033)\end{array}$ \\
\hline Sole establishment & & & $\begin{array}{l}0.099^{* * *} \\
(0.022)\end{array}$ & $\begin{array}{l}0.116^{* * * *} \\
(0.028)\end{array}$ & $\begin{array}{l}0.094^{* * *} \\
(0.022)\end{array}$ & $\begin{array}{l}0.110^{* * *} \\
(0.028)\end{array}$ \\
\hline No. of employees/1000 & & & $\begin{array}{l}-0.045^{* * *} \\
(0.010)\end{array}$ & $\begin{array}{l}-0.050^{* * *} \\
(0.014)\end{array}$ & $\begin{array}{l}-0.039^{* * *} \\
(0.011)\end{array}$ & $\begin{array}{l}-0.041^{* * *} \\
(0.015)\end{array}$ \\
\hline Manufacturing & & & $\begin{array}{l}-0.030 \\
(0.039)\end{array}$ & $\begin{array}{l}-0.038 \\
(0.048)\end{array}$ & $\begin{array}{l}-0.038 \\
(0.040)\end{array}$ & $\begin{array}{l}-0.046 \\
(0.048)\end{array}$ \\
\hline Construction & & & $\begin{array}{l}0.173^{* * * *} \\
(0.064)\end{array}$ & $\begin{array}{l}0.178^{* *} \\
(0.070)\end{array}$ & $\begin{array}{l}0.173^{* * *} \\
(0.064)\end{array}$ & $\begin{array}{l}0.178^{* *} \\
(0.070)\end{array}$ \\
\hline Wholesale and retail trade & & & $\begin{array}{l}0.031 \\
(0.035)\end{array}$ & $\begin{array}{l}0.049 \\
(0.044)\end{array}$ & $\begin{array}{l}0.028 \\
(0.035)\end{array}$ & $\begin{array}{l}0.044 \\
(0.044)\end{array}$ \\
\hline Hotel and restaurant & & & $\begin{array}{l}0.083^{* *} \\
(0.041)\end{array}$ & $\begin{array}{l}0.088^{*} \\
(0.049)\end{array}$ & $\begin{array}{l}0.079^{*} \\
(0.041)\end{array}$ & $\begin{array}{l}0.084^{*} \\
(0.049)\end{array}$ \\
\hline Public and community services & & & $\begin{array}{l}0.103^{* * * *} \\
(0.033)\end{array}$ & $\begin{array}{l}0.118^{* * *} \\
(0.042)\end{array}$ & $\begin{array}{l}0.114^{* * *} \\
(0.034)\end{array}$ & $\begin{array}{l}0.129^{* * *} \\
(0.042)\end{array}$ \\
\hline Education & & & $\begin{array}{l}0.285^{* * * *} \\
(0.037)\end{array}$ & $\begin{array}{l}0.291^{* * * *} \\
(0.048)\end{array}$ & $\begin{array}{l}0.292^{* * *} \\
(0.037)\end{array}$ & $\begin{array}{l}0.298^{* * *} \\
(0.048)\end{array}$ \\
\hline Health & & & $\begin{array}{l}0.285^{* * *} \\
(0.031)\end{array}$ & $\begin{array}{l}0.294^{* * * *} \\
(0.042)\end{array}$ & $\begin{array}{l}0.292^{* * *} \\
(0.032)\end{array}$ & $\begin{array}{l}0.300^{* * * *} \\
(0.042)\end{array}$ \\
\hline Urban area & & & $\begin{array}{l}-0.038^{*} \\
(0.022)\end{array}$ & $\begin{array}{l}-0.054^{*} \\
(0.030)\end{array}$ & $\begin{array}{l}-0.035 \\
(0.022)\end{array}$ & $\begin{array}{l}-0.050^{*} \\
(0.030)\end{array}$ \\
\hline Unemployment to vacancy ratio & & & $\begin{array}{l}-0.011^{* * *} \\
(0.004)\end{array}$ & $\begin{array}{l}-0.013^{* * *} \\
(0.005)\end{array}$ & $\begin{array}{l}-0.010^{* * *} \\
(0.004)\end{array}$ & $\begin{array}{l}-0.012^{* * *} \\
(0.005)\end{array}$ \\
\hline Gender equality policy and practice & & & & & $\begin{array}{l}-0.023^{* *} \\
(0.010)\end{array}$ & $\begin{array}{l}-0.025^{*} \\
(0.013)\end{array}$ \\
\hline Constant & $\begin{array}{l}4.630^{* * * *} \\
(0.071)\end{array}$ & $\begin{array}{l}4.642^{* * * *} \\
(0.075)\end{array}$ & $\begin{array}{l}4.516^{* * *} \\
(0.087)\end{array}$ & $\begin{array}{l}4.566^{* * * *} \\
(0.097)\end{array}$ & $\begin{array}{l}4.516^{* * *} \\
(0.087)\end{array}$ & $\begin{array}{l}4.564^{* * *} \\
(0.097)\end{array}$ \\
\hline $\begin{array}{l}\text { No. of employees } \\
\text { R-squared }\end{array}$ & $\begin{array}{l}9450 \\
0.086\end{array}$ & 9450 & $\begin{array}{l}9450 \\
0.106\end{array}$ & 9450 & $\begin{array}{l}9450 \\
0.106\end{array}$ & 9450 \\
\hline No. of workplaces & & 1409 & & 1409 & & 1409 \\
\hline
\end{tabular}

Robust standard errors in parentheses.

${ }^{* * *} \mathrm{p}<0.01,{ }^{* *} \mathrm{p}<0.05$, and ${ }^{*} \mathrm{p}<0.1$. 
Table 3

Workplace gender diversity and employee job satisfaction, sub-group analysis (male).

\begin{tabular}{|c|c|c|c|c|c|c|}
\hline & OLS & $\mathrm{RE}$ & OLS & $\mathrm{RE}$ & OLS & $\mathrm{RE}$ \\
\hline Gender diversity & $\begin{array}{l}0.061 \\
(0.077)\end{array}$ & $\begin{array}{l}0.013 \\
(0.096)\end{array}$ & $\begin{array}{l}0.102 \\
(0.079)\end{array}$ & $\begin{array}{l}0.074 \\
(0.097)\end{array}$ & $\begin{array}{l}0.105 \\
(0.080)\end{array}$ & $\begin{array}{l}0.082 \\
(0.098)\end{array}$ \\
\hline Majority female (female > 50\%) & $\begin{array}{l}0.069^{* * *} \\
(0.024)\end{array}$ & $\begin{array}{l}0.075^{* * * *} \\
(0.029)\end{array}$ & $\begin{array}{l}0.010 \\
(0.026)\end{array}$ & $\begin{array}{l}0.013 \\
(0.032)\end{array}$ & $\begin{array}{l}0.010 \\
(0.026)\end{array}$ & $\begin{array}{l}0.014 \\
(0.032)\end{array}$ \\
\hline Age $<30$ & $\begin{array}{l}-0.008 \\
(0.031)\end{array}$ & $\begin{array}{l}-0.020 \\
(0.031)\end{array}$ & $\begin{array}{l}0.006 \\
(0.031)\end{array}$ & $\begin{array}{l}-0.009 \\
(0.031)\end{array}$ & $\begin{array}{l}0.006 \\
(0.031)\end{array}$ & $\begin{array}{l}-0.009 \\
(0.031)\end{array}$ \\
\hline Age 30-39 & $\begin{array}{c}-0.028 \\
(0.027)\end{array}$ & $\begin{array}{c}-0.035 \\
(0.026)\end{array}$ & $\begin{array}{c}-0.023 \\
(0.027)\end{array}$ & $\begin{array}{l}-0.031 \\
(0.026)\end{array}$ & $\begin{array}{l}-0.023 \\
(0.027)\end{array}$ & $\begin{array}{l}-0.031 \\
(0.026)\end{array}$ \\
\hline Age $50+$ & $\begin{array}{l}0.148^{* * * *} \\
(0.027)\end{array}$ & $\begin{array}{l}0.132^{* * *} \\
(0.026)\end{array}$ & $\begin{array}{l}0.134^{* * *} \\
(0.027)\end{array}$ & $\begin{array}{l}0.124^{* * *} \\
(0.026)\end{array}$ & $\begin{array}{l}0.134^{* * *} \\
(0.027)\end{array}$ & $\begin{array}{l}0.124^{* * *} \\
(0.026)\end{array}$ \\
\hline Married & $\begin{array}{l}0.078^{* * * *} \\
(0.023)\end{array}$ & $\begin{array}{l}0.070^{* * * *} \\
(0.023)\end{array}$ & $\begin{array}{l}0.079 * * * \\
(0.023)\end{array}$ & $\begin{array}{l}0.072^{* * *} \\
(0.023)\end{array}$ & $\begin{array}{l}0.080^{* * *} \\
(0.023)\end{array}$ & $\begin{array}{l}0.072^{* * * *} \\
(0.023)\end{array}$ \\
\hline White & $\begin{array}{c}-0.070 \\
(0.044)\end{array}$ & $\begin{array}{l}-0.081^{*} \\
(0.044)\end{array}$ & $\begin{array}{l}-0.088^{* *} \\
(0.044)\end{array}$ & $\begin{array}{l}-0.089^{* *} \\
(0.044)\end{array}$ & $\begin{array}{l}-0.088^{* *} \\
(0.044)\end{array}$ & $\begin{array}{l}-0.089^{* *} \\
(0.044)\end{array}$ \\
\hline Children $<7$ years old & $\begin{array}{l}0.052^{* *} \\
(0.026)\end{array}$ & $\begin{array}{l}0.047^{*} \\
(0.025)\end{array}$ & $\begin{array}{l}0.045^{*} \\
(0.026)\end{array}$ & $\begin{array}{l}0.043^{*} \\
(0.025)\end{array}$ & $\begin{array}{l}0.046^{*} \\
(0.026)\end{array}$ & $\begin{array}{l}0.043^{*} \\
(0.025)\end{array}$ \\
\hline Other dependents & $\begin{array}{l}-0.070^{* *} \\
(0.030)\end{array}$ & $\begin{array}{l}-0.073^{* * *} \\
(0.027)\end{array}$ & $\begin{array}{l}-0.074^{* *} \\
(0.029)\end{array}$ & $\begin{array}{l}-0.074^{* * *} \\
(0.027)\end{array}$ & $\begin{array}{l}-0.074^{* *} \\
(0.029)\end{array}$ & $\begin{array}{l}-0.074^{* * *} \\
(0.027)\end{array}$ \\
\hline Disabled & $\begin{array}{l}-0.142^{* * *} \\
(0.030)\end{array}$ & $\begin{array}{l}-0.137^{* * *} \\
(0.028)\end{array}$ & $\begin{array}{l}-0.142^{* * * *} \\
(0.030)\end{array}$ & $\begin{array}{l}-0.138^{* * * *} \\
(0.028)\end{array}$ & $\begin{array}{l}-0.141^{* * * *} \\
(0.030)\end{array}$ & $\begin{array}{l}-0.138^{* * * *} \\
(0.028)\end{array}$ \\
\hline No academic qualification & $\begin{array}{l}0.200^{* * * *} \\
(0.036)\end{array}$ & $\begin{array}{l}0.190^{* * * *} \\
(0.036)\end{array}$ & $\begin{array}{l}0.192^{* * * *} \\
(0.036)\end{array}$ & $\begin{array}{l}0.188^{* * *} \\
(0.036)\end{array}$ & $\begin{array}{l}0.191^{* * * *} \\
(0.036)\end{array}$ & $\begin{array}{l}0.188^{* * *} \\
(0.036)\end{array}$ \\
\hline O-level & $\begin{array}{l}0.069^{* *} \\
(0.030)\end{array}$ & $\begin{array}{l}0.076^{* *} \\
(0.031)\end{array}$ & $\begin{array}{l}0.067^{* *} \\
(0.030)\end{array}$ & $\begin{array}{l}0.077^{* *} \\
(0.031)\end{array}$ & $\begin{array}{l}0.067^{* *} \\
(0.030)\end{array}$ & $\begin{array}{l}0.076^{* *} \\
(0.031)\end{array}$ \\
\hline A-level & $\begin{array}{l}0.023 \\
(0.038)\end{array}$ & $\begin{array}{l}0.044 \\
(0.038)\end{array}$ & $\begin{array}{l}0.039 \\
(0.038)\end{array}$ & $\begin{array}{l}0.055 \\
(0.038)\end{array}$ & $\begin{array}{l}0.039 \\
(0.038)\end{array}$ & $\begin{array}{l}0.055 \\
(0.038)\end{array}$ \\
\hline Other qualification & $\begin{array}{l}0.047^{*} \\
(0.027)\end{array}$ & $\begin{array}{l}0.050^{*} \\
(0.027)\end{array}$ & $\begin{array}{l}0.043 \\
(0.027)\end{array}$ & $\begin{array}{l}0.049^{*} \\
(0.027)\end{array}$ & $\begin{array}{l}0.043 \\
(0.027)\end{array}$ & $\begin{array}{l}0.049^{*} \\
(0.027)\end{array}$ \\
\hline On permanent contract & $\begin{array}{l}0.038 \\
(0.040)\end{array}$ & $\begin{array}{l}0.061 \\
(0.039)\end{array}$ & $\begin{array}{l}0.061 \\
(0.039)\end{array}$ & $\begin{array}{l}0.074^{*} \\
(0.039)\end{array}$ & $\begin{array}{l}0.061 \\
(0.039)\end{array}$ & $\begin{array}{l}0.073^{*} \\
(0.039)\end{array}$ \\
\hline Full-time & $\begin{array}{l}-0.109^{* *} \\
(0.046)\end{array}$ & $\begin{array}{l}-0.103^{* *} \\
(0.044)\end{array}$ & $\begin{array}{l}-0.089^{*} \\
(0.045)\end{array}$ & $\begin{array}{l}-0.090^{* *} \\
(0.044)\end{array}$ & $\begin{array}{l}-0.089^{*} \\
(0.045)\end{array}$ & $\begin{array}{l}-0.090^{* *} \\
(0.044)\end{array}$ \\
\hline Works over $48 \mathrm{~h}$ & $\begin{array}{l}0.073^{* * *} \\
(0.021)\end{array}$ & $\begin{array}{l}0.074^{* * *} \\
(0.021)\end{array}$ & $\begin{array}{l}0.061^{* * *} \\
(0.021)\end{array}$ & $\begin{array}{l}0.067^{* * *} \\
(0.021)\end{array}$ & $\begin{array}{l}0.060 * * * \\
(0.021)\end{array}$ & $\begin{array}{l}0.066^{* * *} \\
(0.021)\end{array}$ \\
\hline Skill same as required & $\begin{array}{l}0.266^{* * * *} \\
(0.019)\end{array}$ & $\begin{array}{l}0.250^{* * * *} \\
(0.019)\end{array}$ & $\begin{array}{l}0.252^{* * * *} \\
(0.018)\end{array}$ & $\begin{array}{l}0.242^{* * * *} \\
(0.019)\end{array}$ & $\begin{array}{l}0.252^{* * *} \\
(0.018)\end{array}$ & $\begin{array}{l}0.242^{* * *} \\
(0.019)\end{array}$ \\
\hline Professional & $\begin{array}{l}-0.242^{* * * *} \\
(0.035)\end{array}$ & $\begin{array}{l}-0.269^{* * * *} \\
(0.036)\end{array}$ & $\begin{array}{l}-0.274^{* * *} \\
(0.035)\end{array}$ & $\begin{array}{l}-0.292^{* * *} \\
(0.037)\end{array}$ & $\begin{array}{l}-0.274^{* * *} \\
(0.035)\end{array}$ & $\begin{array}{l}-0.292^{* * * *} \\
(0.037)\end{array}$ \\
\hline Associate prof. and technical & $\begin{array}{l}-0.222^{* * *} \\
(0.033)\end{array}$ & $\begin{array}{l}-0.242^{* * *} \\
(0.034)\end{array}$ & $\begin{array}{l}-0.235^{* * *} \\
(0.033)\end{array}$ & $\begin{array}{l}-0.249^{* * *} \\
(0.034)\end{array}$ & $\begin{array}{l}-0.235^{* * *} \\
(0.033)\end{array}$ & $\begin{array}{l}-0.249^{* * *} \\
(0.034)\end{array}$ \\
\hline Admin. and secretarial & $\begin{array}{l}-0.353^{* * *} \\
(0.040)\end{array}$ & $\begin{array}{l}-0.332^{* * * *} \\
(0.041)\end{array}$ & $\begin{array}{l}-0.335^{* * *} \\
(0.039)\end{array}$ & $\begin{array}{l}-0.320^{* * * *} \\
(0.041)\end{array}$ & $\begin{array}{l}-0.335^{* * *} \\
(0.039)\end{array}$ & $\begin{array}{l}-0.319^{* * * *} \\
(0.041)\end{array}$ \\
\hline Skilled trades plant and mach. & $\begin{array}{l}-0.364^{* * *} \\
(0.032)\end{array}$ & $\begin{array}{l}-0.359^{* * * *} \\
(0.034)\end{array}$ & $\begin{array}{l}-0.359^{* * *} \\
(0.033)\end{array}$ & $\begin{array}{l}-0.358^{* * * *} \\
(0.034)\end{array}$ & $\begin{array}{l}-0.359^{* * * *} \\
(0.033)\end{array}$ & $\begin{array}{l}-0.359^{* * * *} \\
(0.034)\end{array}$ \\
\hline Personal and customer services & $\begin{array}{l}-0.371^{* * *} \\
(0.047)\end{array}$ & $\begin{array}{l}-0.364^{* * *} \\
(0.047)\end{array}$ & $\begin{array}{l}-0.406^{* * *} \\
(0.047)\end{array}$ & $\begin{array}{l}-0.391^{* * *} \\
(0.047)\end{array}$ & $\begin{array}{l}-0.406^{* * *} \\
(0.047)\end{array}$ & $\begin{array}{l}-0.392^{\text {**** }} \\
(0.047)\end{array}$ \\
\hline Elementary occupations & $\begin{array}{l}-0.389^{* * *} \\
(0.041)\end{array}$ & $\begin{array}{l}-0.380^{* * *} \\
(0.041)\end{array}$ & $\begin{array}{l}-0.365^{* * *} \\
(0.041)\end{array}$ & $\begin{array}{l}-0.365^{\text {**** }} \\
(0.041)\end{array}$ & $\begin{array}{l}-0.365^{* * *} \\
(0.041)\end{array}$ & $\begin{array}{l}-0.365^{\text {**** }} \\
(0.041)\end{array}$ \\
\hline Gross weekly pay $\leq 110$ & $\begin{array}{l}0.080 \\
(0.069)\end{array}$ & $\begin{array}{l}0.039 \\
(0.070)\end{array}$ & $\begin{array}{l}0.028 \\
(0.068)\end{array}$ & $\begin{array}{l}0.002 \\
(0.070)\end{array}$ & $\begin{array}{l}0.027 \\
(0.068)\end{array}$ & $\begin{array}{l}0.000 \\
(0.071)\end{array}$ \\
\hline Gross weekly pay $111-180$ & $\begin{array}{l}0.002 \\
(0.060)\end{array}$ & $\begin{array}{l}-0.022 \\
(0.058)\end{array}$ & $\begin{array}{l}-0.042 \\
(0.061)\end{array}$ & $\begin{array}{l}-0.055 \\
(0.058)\end{array}$ & $\begin{array}{l}-0.042 \\
(0.061)\end{array}$ & $\begin{array}{l}-0.055 \\
(0.058)\end{array}$ \\
\hline Gross weekly pay $181-260$ & $\begin{array}{l}-0.156^{* * *} \\
(0.035)\end{array}$ & $\begin{array}{l}-0.176^{* * *} \\
(0.034)\end{array}$ & $\begin{array}{l}-0.173^{* * *} \\
(0.035)\end{array}$ & $\begin{array}{l}-0.186^{* * *} \\
(0.034)\end{array}$ & $\begin{array}{l}-0.174^{* * *} \\
(0.035)\end{array}$ & $\begin{array}{l}-0.187^{\text {*** }} \\
(0.034)\end{array}$ \\
\hline Gross weekly pay 261-360 & $\begin{array}{l}-0.187^{* * *} \\
(0.027)\end{array}$ & $\begin{array}{l}-0.187^{* * *} \\
(0.027)\end{array}$ & $\begin{array}{l}-0.196^{* * *} \\
(0.027)\end{array}$ & $\begin{array}{l}-0.193^{* * *} \\
(0.027)\end{array}$ & $\begin{array}{l}-0.197^{* * * *} \\
(0.027)\end{array}$ & $\begin{array}{l}-0.194^{* * *} \\
(0.027)\end{array}$ \\
\hline Trade union member & $\begin{array}{l}-0.242^{* * *} \\
(0.021)\end{array}$ & $\begin{array}{l}-0.212^{* * *} \\
(0.022)\end{array}$ & $\begin{array}{l}-0.219^{* * *} \\
(0.023)\end{array}$ & $\begin{array}{l}-0.195^{* * *} \\
(0.024)\end{array}$ & $\begin{array}{l}-0.218^{* * *} \\
(0.024)\end{array}$ & $\begin{array}{l}-0.194^{* * *} \\
(0.024)\end{array}$ \\
\hline Log workplace age & & & $\begin{array}{l}-0.014^{*} \\
(0.008)\end{array}$ & $\begin{array}{l}-0.017 \\
(0.011)\end{array}$ & $\begin{array}{l}-0.014^{*} \\
(0.008)\end{array}$ & $\begin{array}{l}-0.017 \\
(0.011)\end{array}$ \\
\hline Private establishment & & & $\begin{array}{l}0.009 \\
(0.031)\end{array}$ & $\begin{array}{l}0.019 \\
(0.038)\end{array}$ & $\begin{array}{l}0.007 \\
(0.031)\end{array}$ & $\begin{array}{l}0.014 \\
(0.039)\end{array}$ \\
\hline Sole establishment & & & $\begin{array}{l}0.141^{* * *} \\
(0.023)\end{array}$ & $\begin{array}{l}0.152^{* * *} \\
(0.030)\end{array}$ & $\begin{array}{l}0.140^{* * *} \\
(0.023)\end{array}$ & $\begin{array}{l}0.148^{* * *} \\
(0.030)\end{array}$ \\
\hline No. of employees/1000 & & & $\begin{array}{l}-0.042^{* * * *} \\
(0.013)\end{array}$ & $\begin{array}{l}-0.050^{* * *} \\
(0.017)\end{array}$ & $\begin{array}{l}-0.040^{* * *} \\
(0.014)\end{array}$ & $\begin{array}{l}-0.044^{* *} \\
(0.018)\end{array}$ \\
\hline Manufacturing & & & $\begin{array}{l}-0.020 \\
(0.033)\end{array}$ & $\begin{array}{l}-0.018 \\
(0.043)\end{array}$ & $\begin{array}{l}-0.021 \\
(0.033)\end{array}$ & $\begin{array}{l}-0.021 \\
(0.043)\end{array}$ \\
\hline Construction & & & $\begin{array}{l}0.176^{* * *} \\
(0.039)\end{array}$ & $\begin{array}{l}0.169^{* * * *} \\
(0.056)\end{array}$ & $\begin{array}{l}0.176^{* * *} \\
(0.040)\end{array}$ & $\begin{array}{l}0.168^{* * * *} \\
(0.056)\end{array}$ \\
\hline Wholesale and retail trade & & & $\begin{array}{l}0.161^{* * * *} \\
(0.039)\end{array}$ & $\begin{array}{l}0.151^{* * *} \\
(0.050)\end{array}$ & $\begin{array}{l}0.160^{* * *} \\
(0.039)\end{array}$ & $\begin{array}{l}0.148^{* * *} \\
(0.050)\end{array}$ \\
\hline Hotel and restaurant & & & $\begin{array}{l}0.006 \\
(0.039)\end{array}$ & $\begin{array}{l}0.013 \\
(0.048)\end{array}$ & $\begin{array}{l}0.006 \\
(0.039)\end{array}$ & $\begin{array}{l}0.012 \\
(0.048)\end{array}$ \\
\hline Public and community services & & & $\begin{array}{l}0.113^{* * *} \\
(0.036)\end{array}$ & $\begin{array}{l}0.107^{* *} \\
(0.046)\end{array}$ & $\begin{array}{l}0.115^{* * *} \\
(0.037)\end{array}$ & $\begin{array}{l}0.112^{* *} \\
(0.047)\end{array}$ \\
\hline
\end{tabular}


Table 3 (continued)

\begin{tabular}{|c|c|c|c|c|c|c|}
\hline & OLS & $\mathrm{RE}$ & OLS & $\mathrm{RE}$ & OLS & $\mathrm{RE}$ \\
\hline Education & & & $\begin{array}{l}0.273^{* * *} \\
(0.050)\end{array}$ & $\begin{array}{l}0.273^{* * * *} \\
(0.062)\end{array}$ & $\begin{array}{l}0.273^{* * * *} \\
(0.050)\end{array}$ & $\begin{array}{l}0.275^{* * * *} \\
(0.062)\end{array}$ \\
\hline Health & & & $\begin{array}{l}0.251^{* * * *} \\
(0.052)\end{array}$ & $\begin{array}{l}0.265^{* * * *} \\
(0.059)\end{array}$ & $\begin{array}{l}0.251^{* * * *} \\
(0.052)\end{array}$ & $\begin{array}{l}0.266^{* * *} \\
(0.059)\end{array}$ \\
\hline Urban area & & & $\begin{array}{l}-0.076^{* * *} \\
(0.023)\end{array}$ & $\begin{array}{l}-0.066^{* *} \\
(0.031)\end{array}$ & $\begin{array}{l}-0.076^{* * *} \\
(0.023)\end{array}$ & $\begin{array}{l}-0.065^{* *} \\
(0.031)\end{array}$ \\
\hline Unemployment to vacancy ratio & & & $\begin{array}{l}-0.000 \\
(0.004)\end{array}$ & $\begin{array}{l}-0.000 \\
(0.005)\end{array}$ & $\begin{array}{l}0.000 \\
(0.004)\end{array}$ & $\begin{array}{l}-0.000 \\
(0.005)\end{array}$ \\
\hline Gender equality policy and practice & & & & & $\begin{array}{l}-0.004 \\
(0.012)\end{array}$ & $\begin{array}{l}-0.012 \\
(0.015)\end{array}$ \\
\hline Constant & $\begin{array}{l}4.518^{* * * *} \\
(0.084)\end{array}$ & $\begin{array}{l}4.543^{* * * *} \\
(0.086)\end{array}$ & $\begin{array}{l}4.526^{* * *} \\
(0.100)\end{array}$ & $\begin{array}{l}4.531^{* * *} \\
(0.110)\end{array}$ & $\begin{array}{l}4.526^{* * *} \\
(0.100)\end{array}$ & $\begin{array}{l}4.530^{* * *} \\
(0.110)\end{array}$ \\
\hline No. of employees & 8614 & 8614 & 8614 & 8614 & 8614 & 8614 \\
\hline R-squared & 0.101 & & 0.118 & & 0.118 & \\
\hline No. of workplaces & & 1336 & & 1336 & & 1336 \\
\hline
\end{tabular}

Robust standard errors in parentheses.

${ }^{* * *} \mathrm{p}<0.01,{ }^{* *} \mathrm{p}<0.05$, and ${ }^{*} \mathrm{p}<0.1$.

Table 4

Workplace gender diversity and employee job satisfaction, group-size related effect.

\begin{tabular}{|c|c|c|c|c|c|c|}
\hline & OLS & $\mathrm{RE}$ & OLS & RE & OLS & $\mathrm{RE}$ \\
\hline \multirow[t]{2}{*}{ Gender diversity } & $0.164^{* *}$ & $0.179^{*}$ & $0.188^{* *}$ & $0.187^{* *}$ & $0.193^{* * *}$ & $0.196^{* *}$ \\
\hline & $(0.071)$ & $(0.093)$ & $(0.073)$ & $(0.094)$ & $(0.073)$ & $(0.094)$ \\
\hline \multirow[t]{2}{*}{ Majority female (female $>50 \%$ ) } & $0.370^{* * *}$ & $0.385^{* * *}$ & $0.241^{* * *}$ & $0.250^{* * * *}$ & $0.237^{* * * *}$ & $0.245^{* * * *}$ \\
\hline & $(0.038)$ & $(0.048)$ & $(0.044)$ & $(0.055)$ & $(0.044)$ & $(0.055)$ \\
\hline \multirow[t]{2}{*}{ Gender diversity* Majority female } & $-0.766^{* * *}$ & $-0.813^{* * *}$ & $-0.558^{* * *}$ & $-0.587^{* * *}$ & $-0.547^{* * *}$ & $-0.574^{* * *}$ \\
\hline & $(0.093)$ & $(0.125)$ & $(0.100)$ & $(0.130)$ & $(0.100)$ & $(0.130)$ \\
\hline \multirow[t]{2}{*}{ Age $<30$} & $-0.037^{*}$ & $-0.039 * *$ & -0.020 & -0.030 & -0.021 & -0.031 \\
\hline & $(0.020)$ & $(0.019)$ & $(0.020)$ & $(0.019)$ & $(0.020)$ & $(0.019)$ \\
\hline \multirow[t]{2}{*}{ Age 30-39 } & -0.000 & -0.002 & 0.010 & 0.003 & 0.009 & 0.003 \\
\hline & $(0.018)$ & $(0.017)$ & $(0.018)$ & $(0.017)$ & $(0.018)$ & $(0.017)$ \\
\hline \multirow[t]{2}{*}{ Age $50+$} & $0.115^{* * *}$ & $0.104^{* * *}$ & $0.100^{* * *}$ & $0.097^{* * *}$ & $0.100^{* * *}$ & $0.097^{* * * *}$ \\
\hline & $(0.018)$ & $(0.017)$ & $(0.018)$ & $(0.017)$ & $(0.018)$ & $(0.017)$ \\
\hline \multirow[t]{2}{*}{ Female } & $0.067^{* * *}$ & $0.062^{* * *}$ & $0.060^{* * * *}$ & $0.059^{* * * *}$ & $0.060^{* * * *}$ & $0.059^{* * *}$ \\
\hline & $(0.016)$ & $(0.016)$ & $(0.016)$ & $(0.016)$ & $(0.016)$ & $(0.016)$ \\
\hline \multirow[t]{2}{*}{ Married } & $0.059^{* * *}$ & $0.052^{* * *}$ & $0.059^{* * *}$ & $0.053^{* * *}$ & $0.059^{* * *}$ & $0.053^{* * *}$ \\
\hline & $(0.015)$ & $(0.014)$ & $(0.014)$ & $(0.014)$ & $(0.014)$ & $(0.014)$ \\
\hline \multirow[t]{2}{*}{ White } & -0.021 & -0.034 & -0.038 & -0.046 & -0.039 & -0.046 \\
\hline & $(0.029)$ & $(0.029)$ & $(0.029)$ & $(0.029)$ & $(0.029)$ & $(0.029)$ \\
\hline \multirow[t]{2}{*}{ Children $<7$ years old } & 0.015 & 0.021 & 0.013 & 0.020 & 0.014 & 0.020 \\
\hline & $(0.018)$ & $(0.018)$ & $(0.018)$ & $(0.018)$ & $(0.018)$ & $(0.018)$ \\
\hline \multirow[t]{2}{*}{ Other dependents } & $-0.062^{* * *}$ & $-0.065^{* * *}$ & $-0.066^{* * *}$ & $-0.066^{* * *}$ & $-0.065^{* * *}$ & $-0.066^{* * *}$ \\
\hline & $(0.018)$ & $(0.017)$ & $(0.018)$ & $(0.017)$ & $(0.018)$ & $(0.017)$ \\
\hline \multirow[t]{2}{*}{ Disabled } & $-0.123^{* * *}$ & $-0.112^{* * *}$ & $-0.117^{* * *}$ & $-0.110^{* * *}$ & $-0.117^{* * *}$ & $-0.110^{* * *}$ \\
\hline & $(0.021)$ & $(0.019)$ & $(0.021)$ & $(0.019)$ & $(0.021)$ & $(0.019)$ \\
\hline \multirow[t]{2}{*}{ No academic qualification } & $0.153^{* * *}$ & $0.151^{* * *}$ & $0.162^{* * *}$ & $0.155^{* * *}$ & $0.160^{* * *}$ & $0.154^{* * *}$ \\
\hline & $(0.026)$ & $(0.025)$ & $(0.026)$ & $(0.025)$ & $(0.026)$ & $(0.025)$ \\
\hline \multirow[t]{2}{*}{ O-level } & $0.080^{* * *}$ & $0.089^{* * *}$ & $0.090^{* * *}$ & $0.093^{* * *}$ & $0.089^{* * *}$ & $0.092^{* * *}$ \\
\hline & $(0.021)$ & $(0.021)$ & $(0.021)$ & $(0.021)$ & $(0.021)$ & $(0.021)$ \\
\hline \multirow[t]{2}{*}{ A-level } & -0.003 & 0.024 & 0.014 & 0.031 & 0.014 & 0.031 \\
\hline & $(0.026)$ & $(0.025)$ & $(0.026)$ & $(0.025)$ & $(0.026)$ & $(0.025)$ \\
\hline \multirow[t]{2}{*}{ Other qualification } & $0.062^{* * *}$ & $0.062^{* * *}$ & $0.061^{* * *}$ & $0.061^{* * * *}$ & $0.060^{* * * *}$ & $0.060^{* * * *}$ \\
\hline & $(0.018)$ & $(0.018)$ & $(0.018)$ & $(0.018)$ & $(0.018)$ & $(0.018)$ \\
\hline \multirow[t]{2}{*}{ On permanent contract } & 0.013 & 0.036 & $0.040^{*}$ & $0.048^{* *}$ & 0.039 & $0.048^{* *}$ \\
\hline & $(0.024)$ & $(0.024)$ & $(0.024)$ & $(0.024)$ & $(0.024)$ & $(0.024)$ \\
\hline \multirow[t]{2}{*}{ Full-time } & -0.033 & -0.034 & -0.031 & -0.035 & -0.032 & -0.035 \\
\hline & $(0.022)$ & $(0.022)$ & $(0.022)$ & $(0.022)$ & $(0.022)$ & $(0.022)$ \\
\hline \multirow[t]{2}{*}{ Works over $48 \mathrm{~h}$} & $0.062^{* * *}$ & $0.053^{* * *}$ & $0.047^{* * * *}$ & $0.045^{* * * *}$ & $0.046^{* * *}$ & $0.045^{* * *}$ \\
\hline & $(0.015)$ & $(0.015)$ & $(0.015)$ & $(0.014)$ & $(0.015)$ & $(0.015)$ \\
\hline \multirow[t]{2}{*}{ Skill same as required } & $0.276^{* * *}$ & $0.259^{* * *}$ & $0.270^{* * * *}$ & $0.257^{* * * *}$ & $0.270^{* * * *}$ & $0.257^{* * *}$ \\
\hline & $(0.012)$ & $(0.012)$ & $(0.012)$ & $(0.012)$ & $(0.012)$ & $(0.012)$ \\
\hline \multirow[t]{2}{*}{ Professional } & $-0.205^{* * *}$ & $-0.237^{* * *}$ & $-0.242^{* * *}$ & $-0.255^{* * *}$ & $-0.243^{* * *}$ & $-0.255^{* * *}$ \\
\hline & $(0.025)$ & $(0.027)$ & $(0.026)$ & $(0.027)$ & $(0.026)$ & $(0.027)$ \\
\hline Associate prof. and technical & $-0.206^{* * *}$ & $-0.226^{* * *}$ & $-0.216^{* * *}$ & $-0.228^{* * *}$ & $-0.216^{* * *}$ & $-0.228^{* * *}$ \\
\hline & $(0.024)$ & $(0.024)$ & $(0.024)$ & $(0.024)$ & $(0.024)$ & $(0.024)$ \\
\hline Admin. and secretarial & $-0.350^{* * *}$ & $-0.326^{* * *}$ & $-0.335^{* * * *}$ & $-0.317^{* * *}$ & $-0.335^{* * *}$ & $-0.317^{* * *}$ \\
\hline & $(0.025)$ & $(0.025)$ & $(0.025)$ & $(0.025)$ & $(0.025)$ & $(0.025)$ \\
\hline Skilled trades plant and mach. & $-0.370^{* * *}$ & $-0.362^{* * *}$ & $-0.369^{* * *}$ & $-0.363^{* * *}$ & $-0.370^{* * *}$ & $-0.364^{* * *}$ \\
\hline & $(0.027)$ & $(0.027)$ & $(0.027)$ & $(0.028)$ & $(0.027)$ & $(0.028)$ \\
\hline
\end{tabular}


Table 4 (continued)

\begin{tabular}{|c|c|c|c|c|c|c|}
\hline & OLS & $\mathrm{RE}$ & OLS & RE & OLS & $\mathrm{RE}$ \\
\hline Personal and customer services & $\begin{array}{l}-0.308^{* * *} \\
(0.027)\end{array}$ & $\begin{array}{l}-0.291^{* * *} \\
(0.028)\end{array}$ & $\begin{array}{l}-0.329^{* * *} \\
(0.027)\end{array}$ & $\begin{array}{l}-0.303^{* * *} \\
(0.028)\end{array}$ & $\begin{array}{l}-0.329^{* * *} \\
(0.027)\end{array}$ & $\begin{array}{l}-0.303^{* * *} \\
(0.028)\end{array}$ \\
\hline Elementary occupations & $\begin{array}{l}-0.347^{* * *} \\
(0.030)\end{array}$ & $\begin{array}{l}-0.337^{* * * *} \\
(0.030)\end{array}$ & $\begin{array}{l}-0.337^{* * * *} \\
(0.030)\end{array}$ & $\begin{array}{l}-0.330^{* * * *} \\
(0.030)\end{array}$ & $\begin{array}{l}-0.337^{* * *} \\
(0.030)\end{array}$ & $\begin{array}{l}-0.330^{\text {*** }} \\
(0.030)\end{array}$ \\
\hline Gross weekly pay $\leq 110$ & $\begin{array}{l}-0.059^{*} \\
(0.034)\end{array}$ & $\begin{array}{l}-0.121^{* * *} \\
(0.035)\end{array}$ & $\begin{array}{l}-0.094^{* * *} \\
(0.034)\end{array}$ & $\begin{array}{l}-0.142^{* * *} \\
(0.035)\end{array}$ & $\begin{array}{l}-0.096^{* * *} \\
(0.034)\end{array}$ & $\begin{array}{l}-0.144^{* * *} \\
(0.035)\end{array}$ \\
\hline Gross weekly pay $111-180$ & $\begin{array}{l}-0.110^{* * *} \\
(0.030)\end{array}$ & $\begin{array}{l}-0.156^{* * * *} \\
(0.029)\end{array}$ & $\begin{array}{l}-0.146^{* * * *} \\
(0.030)\end{array}$ & $\begin{array}{l}-0.177^{* * * *} \\
(0.029)\end{array}$ & $\begin{array}{l}-0.148^{* * *} \\
(0.030)\end{array}$ & $\begin{array}{l}-0.179^{* * *} \\
(0.029)\end{array}$ \\
\hline Gross weekly pay $181-260$ & $\begin{array}{l}-0.131^{* * *} \\
(0.022)\end{array}$ & $\begin{array}{l}-0.155^{* * *} \\
(0.022)\end{array}$ & $\begin{array}{l}-0.155^{* * *} \\
(0.022)\end{array}$ & $\begin{array}{l}-0.168^{* * *} \\
(0.022)\end{array}$ & $\begin{array}{l}-0.156^{* * *} \\
(0.022)\end{array}$ & $\begin{array}{l}-0.169^{* * *} \\
(0.022)\end{array}$ \\
\hline Gross weekly pay $261-360$ & $\begin{array}{l}-0.139^{* * *} \\
(0.018)\end{array}$ & $\begin{array}{l}-0.148^{* * *} \\
(0.018)\end{array}$ & $\begin{array}{l}-0.153^{* * *} \\
(0.018)\end{array}$ & $\begin{array}{l}-0.155^{* * * *} \\
(0.018)\end{array}$ & $\begin{array}{l}-0.154^{* * *} \\
(0.018)\end{array}$ & $\begin{array}{l}-0.156^{* * *} \\
(0.018)\end{array}$ \\
\hline Trade union member & $\begin{array}{l}-0.202^{* * *} \\
(0.014)\end{array}$ & $\begin{array}{l}-0.173^{* * *} \\
(0.015)\end{array}$ & $\begin{array}{l}-0.176^{* * *} \\
(0.015)\end{array}$ & $\begin{array}{l}-0.155^{* * * *} \\
(0.016)\end{array}$ & $\begin{array}{l}-0.174^{* * *} \\
(0.015)\end{array}$ & $\begin{array}{l}-0.153^{* * *} \\
(0.016)\end{array}$ \\
\hline Log workplace age & & & $\begin{array}{l}-0.019^{* * *} \\
(0.006)\end{array}$ & $\begin{array}{l}-0.019^{* *} \\
(0.008)\end{array}$ & $\begin{array}{l}-0.019^{* * *} \\
(0.006)\end{array}$ & $\begin{array}{l}-0.019^{* *} \\
(0.008)\end{array}$ \\
\hline Private establishment & & & $\begin{array}{l}0.073^{* * *} \\
(0.019)\end{array}$ & $\begin{array}{l}0.084^{* * *} \\
(0.026)\end{array}$ & $\begin{array}{l}0.068^{* * * *} \\
(0.019)\end{array}$ & $\begin{array}{l}0.077^{* * * *} \\
(0.026)\end{array}$ \\
\hline Sole establishment & & & $\begin{array}{l}0.124^{* * *} \\
(0.016)\end{array}$ & $\begin{array}{l}0.141^{* * *} \\
(0.022)\end{array}$ & $\begin{array}{l}0.120^{* * *} \\
(0.016)\end{array}$ & $\begin{array}{l}0.135^{* * *} \\
(0.022)\end{array}$ \\
\hline No. of employees/1000 & & & $\begin{array}{l}-0.044^{* * *} \\
(0.008)\end{array}$ & $\begin{array}{l}-0.047^{* * * *} \\
(0.012)\end{array}$ & $\begin{array}{l}-0.039^{* * *} \\
(0.009)\end{array}$ & $\begin{array}{l}-0.039^{\text {*** }} \\
(0.012)\end{array}$ \\
\hline Manufacturing & & & $\begin{array}{l}-0.025 \\
(0.025)\end{array}$ & $\begin{array}{l}-0.027 \\
(0.034)\end{array}$ & $\begin{array}{l}-0.029 \\
(0.025)\end{array}$ & $\begin{array}{l}-0.032 \\
(0.034)\end{array}$ \\
\hline Construction & & & $\begin{array}{l}0.199^{* * *} \\
(0.033)\end{array}$ & $\begin{array}{l}0.194^{* * * *} \\
(0.047)\end{array}$ & $\begin{array}{l}0.198^{* * *} \\
(0.033)\end{array}$ & $\begin{array}{l}0.193^{* * * *} \\
(0.047)\end{array}$ \\
\hline Wholesale and retail trade & & & $\begin{array}{l}0.101^{* * *} \\
(0.026)\end{array}$ & $\begin{array}{l}0.107^{* * *} \\
(0.035)\end{array}$ & $\begin{array}{l}0.098^{* * *} \\
(0.026)\end{array}$ & $\begin{array}{l}0.103^{* * *} \\
(0.035)\end{array}$ \\
\hline Hotel and restaurant & & & $\begin{array}{l}0.044 \\
(0.028)\end{array}$ & $\begin{array}{l}0.039 \\
(0.037)\end{array}$ & $\begin{array}{l}0.042 \\
(0.028)\end{array}$ & $\begin{array}{l}0.036 \\
(0.037)\end{array}$ \\
\hline Public and community services & & & $\begin{array}{l}0.110^{* * *} \\
(0.024)\end{array}$ & $\begin{array}{l}0.118^{* * *} \\
(0.033)\end{array}$ & $\begin{array}{l}0.116^{* * * *} \\
(0.025)\end{array}$ & $\begin{array}{l}0.127^{* * *} \\
(0.034)\end{array}$ \\
\hline Education & & & $\begin{array}{l}0.276^{* * *} \\
(0.029)\end{array}$ & $\begin{array}{l}0.281^{* * * *} \\
(0.041)\end{array}$ & $\begin{array}{l}0.280^{\text {**** }} \\
(0.029)\end{array}$ & $\begin{array}{l}0.287^{* * *} \\
(0.041)\end{array}$ \\
\hline Health & & & $\begin{array}{l}0.262^{* * *} \\
(0.026)\end{array}$ & $\begin{array}{l}0.267^{* * *} \\
(0.036)\end{array}$ & $\begin{array}{l}0.265^{* * *} \\
(0.026)\end{array}$ & $\begin{array}{l}0.271^{* * *} \\
(0.036)\end{array}$ \\
\hline Urban area & & & $\begin{array}{l}-0.061^{* * * *} \\
(0.016)\end{array}$ & $\begin{array}{l}-0.063^{* * * *} \\
(0.023)\end{array}$ & $\begin{array}{l}-0.060^{* * * *} \\
(0.016)\end{array}$ & $\begin{array}{l}-0.060^{* * *} \\
(0.023)\end{array}$ \\
\hline Unemployment to vacancy ratio & & & $\begin{array}{l}-0.006^{* *} \\
(0.003)\end{array}$ & $\begin{array}{l}-0.008^{* *} \\
(0.004)\end{array}$ & $\begin{array}{l}-0.005^{* *} \\
(0.003)\end{array}$ & $\begin{array}{l}-0.007^{*} \\
(0.004)\end{array}$ \\
\hline Gender equality policy and practice & & & & & $\begin{array}{l}-0.014^{*} \\
(0.008)\end{array}$ & $\begin{array}{l}-0.020^{*} \\
(0.011)\end{array}$ \\
\hline Constant & $\begin{array}{l}4.393^{* * *} \\
(0.056)\end{array}$ & $\begin{array}{l}4.410^{* * * *} \\
(0.060)\end{array}$ & $\begin{array}{l}4.383^{* * *} \\
(0.067)\end{array}$ & $\begin{array}{l}4.397^{* * * *} \\
(0.078)\end{array}$ & $\begin{array}{l}4.385^{* * *} \\
(0.067)\end{array}$ & $\begin{array}{l}4.397^{\text {**** }} \\
(0.078)\end{array}$ \\
\hline $\begin{array}{l}\text { No. of employees } \\
\text { R-squared }\end{array}$ & $\begin{array}{l}18,064 \\
0.094\end{array}$ & 18,064 & $\begin{array}{l}18,064 \\
0.111\end{array}$ & 18,064 & $\begin{array}{l}18,064 \\
0.111\end{array}$ & 18,064 \\
\hline No. of workplaces & & 1506 & & 1506 & & 1506 \\
\hline
\end{tabular}

Robust standard errors in parentheses.

${ }^{* * *} \mathrm{p}<0.01,{ }^{* *} \mathrm{p}<0.05$, and ${ }^{*} \mathrm{p}<0.1$.

Table 5

Workplace gender diversity and affective well-being, main effect.

\begin{tabular}{|c|c|c|c|c|c|c|}
\hline & OLS & $\mathrm{RE}$ & OLS & RE & OLS & $\mathrm{RE}$ \\
\hline Gender diversity & $\begin{array}{l}-0.122^{* *} \\
(0.048)\end{array}$ & $\begin{array}{l}-0.146^{* *} \\
(0.060)\end{array}$ & $\begin{array}{l}-0.106^{* *} \\
(0.051)\end{array}$ & $\begin{array}{l}-0.129^{* *} \\
(0.062)\end{array}$ & $\begin{array}{l}-0.095^{*} \\
(0.051)\end{array}$ & $\begin{array}{l}-0.115^{*} \\
(0.063)\end{array}$ \\
\hline Majority female (female $>50 \%$ ) & $\begin{array}{l}-0.035^{* *} \\
(0.016)\end{array}$ & $\begin{array}{l}-0.036^{*} \\
(0.020)\end{array}$ & $\begin{array}{l}-0.037^{* *} \\
(0.018)\end{array}$ & $\begin{array}{l}-0.039^{*} \\
(0.023)\end{array}$ & $\begin{array}{l}-0.037^{* *} \\
(0.018)\end{array}$ & $\begin{array}{l}-0.039^{*} \\
(0.023)\end{array}$ \\
\hline Age $<30$ & $\begin{array}{l}-0.029 \\
(0.022)\end{array}$ & $\begin{array}{l}-0.028 \\
(0.021)\end{array}$ & $\begin{array}{l}-0.026 \\
(0.022)\end{array}$ & $\begin{array}{l}-0.027 \\
(0.022)\end{array}$ & $\begin{array}{l}-0.027 \\
(0.022)\end{array}$ & $\begin{array}{l}-0.027 \\
(0.022)\end{array}$ \\
\hline Age 30-39 & $\begin{array}{l}-0.005 \\
(0.020)\end{array}$ & $\begin{array}{l}-0.004 \\
(0.019)\end{array}$ & $\begin{array}{l}-0.001 \\
(0.020)\end{array}$ & $\begin{array}{l}-0.001 \\
(0.019)\end{array}$ & $\begin{array}{l}-0.001 \\
(0.020)\end{array}$ & $\begin{array}{l}-0.002 \\
(0.019)\end{array}$ \\
\hline Age $50+$ & $\begin{array}{l}0.140^{* * * *} \\
(0.020)\end{array}$ & $\begin{array}{l}0.128^{* * *} \\
(0.019)\end{array}$ & $\begin{array}{l}0.134^{* * * *} \\
(0.020)\end{array}$ & $\begin{array}{l}0.124^{* * *} \\
(0.019)\end{array}$ & $\begin{array}{l}0.134^{* * *} \\
(0.020)\end{array}$ & $\begin{array}{l}0.124^{* * *} \\
(0.019)\end{array}$ \\
\hline Female & $\begin{array}{l}-0.085^{* * * *} \\
(0.017)\end{array}$ & $\begin{array}{l}-0.080^{* * *} \\
(0.017)\end{array}$ & $\begin{array}{l}-0.085^{* * *} \\
(0.017)\end{array}$ & $\begin{array}{l}-0.080^{* * *} \\
(0.017)\end{array}$ & $\begin{array}{l}-0.085^{* * * *} \\
(0.017)\end{array}$ & $\begin{array}{l}-0.080^{* * *} \\
(0.017)\end{array}$ \\
\hline Married & $\begin{array}{l}-0.005 \\
(0.016)\end{array}$ & $\begin{array}{l}-0.008 \\
(0.016)\end{array}$ & $\begin{array}{l}-0.005 \\
(0.016)\end{array}$ & $\begin{array}{l}-0.008 \\
(0.016)\end{array}$ & $\begin{array}{l}-0.005 \\
(0.016)\end{array}$ & $\begin{array}{l}-0.008 \\
(0.016)\end{array}$ \\
\hline White & $\begin{array}{c}-0.023 \\
(0.032)\end{array}$ & $\begin{array}{l}-0.026 \\
(0.032)\end{array}$ & $\begin{array}{l}-0.036 \\
(0.032)\end{array}$ & $\begin{array}{c}-0.038 \\
(0.032)\end{array}$ & $\begin{array}{c}-0.037 \\
(0.032)\end{array}$ & $\begin{array}{c}-0.039 \\
(0.032)\end{array}$ \\
\hline Children $<7$ year old & $\begin{array}{l}-0.004 \\
(0.020)\end{array}$ & $\begin{array}{l}-0.002 \\
(0.020)\end{array}$ & $\begin{array}{l}-0.005 \\
(0.020)\end{array}$ & $\begin{array}{l}-0.003 \\
(0.020)\end{array}$ & $\begin{array}{l}-0.004 \\
(0.020)\end{array}$ & $\begin{array}{l}-0.002 \\
(0.020)\end{array}$ \\
\hline Other dependents & $\begin{array}{l}-0.105^{* * *} \\
(0.019)\end{array}$ & $\begin{array}{l}-0.106^{* * *} \\
(0.019)\end{array}$ & $\begin{array}{l}-0.105^{* * *} \\
(0.019)\end{array}$ & $\begin{array}{l}-0.106^{* * *} \\
(0.019)\end{array}$ & $\begin{array}{l}-0.104^{* * *} \\
(0.019)\end{array}$ & $\begin{array}{l}-0.105^{* * *} \\
(0.019)\end{array}$ \\
\hline
\end{tabular}


Table 5 (continued)

\begin{tabular}{|c|c|c|c|c|c|c|}
\hline & OLS & $\mathrm{RE}$ & OLS & $\mathrm{RE}$ & OLS & $\mathrm{RE}$ \\
\hline Disabled & $\begin{array}{l}-0.178^{* * * *} \\
(0.022)\end{array}$ & $\begin{array}{l}-0.170^{* * * *} \\
(0.021)\end{array}$ & $\begin{array}{l}-0.174^{* * *} \\
(0.022)\end{array}$ & $\begin{array}{l}-0.169^{* * * *} \\
(0.021)\end{array}$ & $\begin{array}{l}-0.174^{* * * *} \\
(0.022)\end{array}$ & $\begin{array}{l}-0.168^{* * *} \\
(0.021)\end{array}$ \\
\hline No academic qualification & $\begin{array}{l}0.134^{* * * *} \\
(0.028)\end{array}$ & $\begin{array}{l}0.132^{* * * *} \\
(0.028)\end{array}$ & $\begin{array}{l}0.143^{* * * *} \\
(0.029)\end{array}$ & $\begin{array}{l}0.137^{* * * *} \\
(0.028)\end{array}$ & $\begin{array}{l}0.141^{* * * *} \\
(0.029)\end{array}$ & $\begin{array}{l}0.137^{* * * *} \\
(0.028)\end{array}$ \\
\hline O-level & $\begin{array}{l}0.082^{* * *} \\
(0.023)\end{array}$ & $\begin{array}{l}0.081^{* * *} \\
(0.023)\end{array}$ & $\begin{array}{l}0.089^{* * *} \\
(0.023)\end{array}$ & $\begin{array}{l}0.085^{* * *} \\
(0.023)\end{array}$ & $\begin{array}{l}0.088^{* * *} \\
(0.023)\end{array}$ & $\begin{array}{l}0.085^{* * *} \\
(0.023)\end{array}$ \\
\hline A-level & $\begin{array}{l}0.026 \\
(0.027)\end{array}$ & $\begin{array}{l}0.031 \\
(0.028)\end{array}$ & $\begin{array}{l}0.031 \\
(0.027)\end{array}$ & $\begin{array}{l}0.034 \\
(0.028)\end{array}$ & $\begin{array}{l}0.031 \\
(0.027)\end{array}$ & $\begin{array}{l}0.034 \\
(0.028)\end{array}$ \\
\hline Other qualification & $\begin{array}{l}0.036^{*} \\
(0.019)\end{array}$ & $\begin{array}{l}0.035^{*} \\
(0.020)\end{array}$ & $\begin{array}{l}0.039^{* *} \\
(0.020)\end{array}$ & $\begin{array}{l}0.037^{*} \\
(0.020)\end{array}$ & $\begin{array}{l}0.039^{* *} \\
(0.020)\end{array}$ & $\begin{array}{l}0.037^{*} \\
(0.020)\end{array}$ \\
\hline On permanent contract & $\begin{array}{l}-0.080^{* * *} \\
(0.026)\end{array}$ & $\begin{array}{l}-0.066^{* *} \\
(0.026)\end{array}$ & $\begin{array}{l}-0.072^{* * *} \\
(0.026)\end{array}$ & $\begin{array}{l}-0.061^{* *} \\
(0.027)\end{array}$ & $\begin{array}{l}-0.073^{* * *} \\
(0.026)\end{array}$ & $\begin{array}{l}-0.061^{* *} \\
(0.027)\end{array}$ \\
\hline Full-time & $\begin{array}{l}-0.109^{* * *} \\
(0.024)\end{array}$ & $\begin{array}{l}-0.105^{* * *} \\
(0.024)\end{array}$ & $\begin{array}{l}-0.110^{* * * *} \\
(0.024)\end{array}$ & $\begin{array}{l}-0.105^{* * *} \\
(0.024)\end{array}$ & $\begin{array}{l}-0.110^{* * *} \\
(0.024)\end{array}$ & $\begin{array}{l}-0.106^{* * *} \\
(0.024)\end{array}$ \\
\hline Works over $48 \mathrm{~h}$ & $\begin{array}{l}-0.204^{* * *} \\
(0.016)\end{array}$ & $\begin{array}{l}-0.209^{* * *} \\
(0.016)\end{array}$ & $\begin{array}{l}-0.214^{* * *} \\
(0.016)\end{array}$ & $\begin{array}{l}-0.215^{* * *} \\
(0.016)\end{array}$ & $\begin{array}{l}-0.214^{* * *} \\
(0.016)\end{array}$ & $\begin{array}{l}-0.215^{* * *} \\
(0.016)\end{array}$ \\
\hline Skill same as required & $\begin{array}{l}0.063^{* * * *} \\
(0.014)\end{array}$ & $\begin{array}{l}0.059^{* * * *} \\
(0.014)\end{array}$ & $\begin{array}{l}0.062^{* * * *} \\
(0.014)\end{array}$ & $\begin{array}{l}0.059^{* * * *} \\
(0.014)\end{array}$ & $\begin{array}{l}0.062^{* * * *} \\
(0.014)\end{array}$ & $\begin{array}{l}0.058^{* * *} \\
(0.014)\end{array}$ \\
\hline Professional & $\begin{array}{l}-0.031 \\
(0.027)\end{array}$ & $\begin{array}{l}-0.023 \\
(0.029)\end{array}$ & $\begin{array}{l}-0.029 \\
(0.028)\end{array}$ & $\begin{array}{l}-0.023 \\
(0.030)\end{array}$ & $\begin{array}{l}-0.030 \\
(0.028)\end{array}$ & $\begin{array}{l}-0.023 \\
(0.030)\end{array}$ \\
\hline Associate prof. and technical & $\begin{array}{l}0.064^{* *} \\
(0.025)\end{array}$ & $\begin{array}{l}0.054^{* *} \\
(0.027)\end{array}$ & $\begin{array}{l}0.054^{* *} \\
(0.026)\end{array}$ & $\begin{array}{l}0.049^{*} \\
(0.027)\end{array}$ & $\begin{array}{l}0.054^{* *} \\
(0.026)\end{array}$ & $\begin{array}{l}0.049 * \\
(0.027)\end{array}$ \\
\hline Admin. and secretarial & $\begin{array}{l}0.103^{* * * *} \\
(0.027)\end{array}$ & $\begin{array}{l}0.106^{* * *} \\
(0.028)\end{array}$ & $\begin{array}{l}0.103^{* * *} \\
(0.027)\end{array}$ & $\begin{array}{l}0.107^{* * *} \\
(0.028)\end{array}$ & $\begin{array}{l}0.103^{* * *} \\
(0.027)\end{array}$ & $\begin{array}{l}0.107^{* * *} \\
(0.028)\end{array}$ \\
\hline Skilled trades plant and mach. & $\begin{array}{l}0.224^{* * * *} \\
(0.029)\end{array}$ & $\begin{array}{l}0.232^{* * * *} \\
(0.030)\end{array}$ & $\begin{array}{l}0.223^{* * * *} \\
(0.030)\end{array}$ & $\begin{array}{l}0.232^{* * * *} \\
(0.030)\end{array}$ & $\begin{array}{l}0.223^{* * * *} \\
(0.030)\end{array}$ & $\begin{array}{l}0.231^{* * * *} \\
(0.030)\end{array}$ \\
\hline Personal and customer services & $\begin{array}{l}0.114^{* * *} \\
(0.030)\end{array}$ & $\begin{array}{l}0.116^{\text {**** }} \\
(0.031)\end{array}$ & $\begin{array}{l}0.098^{* * *} \\
(0.030)\end{array}$ & $\begin{array}{l}0.106^{* * *} \\
(0.031)\end{array}$ & $\begin{array}{l}0.098^{* * * *} \\
(0.030)\end{array}$ & $\begin{array}{l}0.106^{* * *} \\
(0.031)\end{array}$ \\
\hline Elementary occupations & $\begin{array}{l}0.215^{* * *} \\
(0.033)\end{array}$ & $\begin{array}{l}0.207^{\text {*** }} \\
(0.033)\end{array}$ & $\begin{array}{l}0.218^{* * *} \\
(0.033)\end{array}$ & $\begin{array}{l}0.212^{* * *} \\
(0.033)\end{array}$ & $\begin{array}{l}0.217^{* * *} \\
(0.033)\end{array}$ & $\begin{array}{l}0.212^{\text {*** }} \\
(0.033)\end{array}$ \\
\hline Gross weekly pay $\leq 110$ & $\begin{array}{l}0.323^{* * *} \\
(0.037)\end{array}$ & $\begin{array}{l}0.319^{* * *} \\
(0.038)\end{array}$ & $\begin{array}{l}0.298^{* * *} \\
(0.038)\end{array}$ & $\begin{array}{l}0.300^{* * *} \\
(0.038)\end{array}$ & $\begin{array}{l}0.295^{* * *} \\
(0.038)\end{array}$ & $\begin{array}{l}0.297^{* * *} \\
(0.038)\end{array}$ \\
\hline Gross weekly pay $111-180$ & $\begin{array}{l}0.194^{* * * *} \\
(0.033)\end{array}$ & $\begin{array}{l}0.191^{* * *} \\
(0.032)\end{array}$ & $\begin{array}{l}0.177^{* * *} \\
(0.033)\end{array}$ & $\begin{array}{l}0.177^{* * *} \\
(0.032)\end{array}$ & $\begin{array}{l}0.174^{* * * *} \\
(0.033)\end{array}$ & $\begin{array}{l}0.175^{* * * *} \\
(0.032)\end{array}$ \\
\hline Gross weekly pay $181-260$ & $\begin{array}{l}0.136^{* * *} \\
(0.024)\end{array}$ & $\begin{array}{l}0.143^{* * *} \\
(0.024)\end{array}$ & $\begin{array}{l}0.127^{* * *} \\
(0.024)\end{array}$ & $\begin{array}{l}0.136^{* * *} \\
(0.024)\end{array}$ & $\begin{array}{l}0.125^{* * * *} \\
(0.024)\end{array}$ & $\begin{array}{l}0.135^{* * *} \\
(0.024)\end{array}$ \\
\hline Gross weekly pay $261-360$ & $\begin{array}{l}0.030 \\
(0.020)\end{array}$ & $\begin{array}{l}0.035^{*} \\
(0.020)\end{array}$ & $\begin{array}{l}0.028 \\
(0.020)\end{array}$ & $\begin{array}{l}0.033 \\
(0.020)\end{array}$ & $\begin{array}{l}0.027 \\
(0.020)\end{array}$ & $\begin{array}{l}0.032 \\
(0.020)\end{array}$ \\
\hline Trade union member & $\begin{array}{l}-0.144^{* * *} \\
(0.015)\end{array}$ & $\begin{array}{l}-0.138^{* * *} \\
(0.016)\end{array}$ & $\begin{array}{l}-0.135^{* * *} \\
(0.017)\end{array}$ & $\begin{array}{l}-0.131^{* * *} \\
(0.017)\end{array}$ & $\begin{array}{l}-0.133^{* * *} \\
(0.017)\end{array}$ & $\begin{array}{l}-0.130^{* * *} \\
(0.017)\end{array}$ \\
\hline Log workplace age & & & $\begin{array}{l}-0.013^{* *} \\
(0.006)\end{array}$ & $\begin{array}{l}-0.012 \\
(0.008)\end{array}$ & $\begin{array}{l}-0.013^{* *} \\
(0.006)\end{array}$ & $\begin{array}{l}-0.012 \\
(0.008)\end{array}$ \\
\hline Private establishment & & & $\begin{array}{l}0.086^{* * *} \\
(0.021)\end{array}$ & $\begin{array}{l}0.084^{* * * *} \\
(0.026)\end{array}$ & $\begin{array}{l}0.081^{* * *} \\
(0.021)\end{array}$ & $\begin{array}{l}0.078^{* * *} \\
(0.027)\end{array}$ \\
\hline Sole establishment & & & $\begin{array}{l}0.034^{*} \\
(0.018)\end{array}$ & $\begin{array}{l}0.042^{*} \\
(0.022)\end{array}$ & $\begin{array}{l}0.030^{*} \\
(0.018)\end{array}$ & $\begin{array}{l}0.037^{*} \\
(0.022)\end{array}$ \\
\hline No. of employees/1000 & & & $\begin{array}{l}-0.001 \\
(0.009)\end{array}$ & $\begin{array}{l}-0.000 \\
(0.012)\end{array}$ & $\begin{array}{l}0.005 \\
(0.009)\end{array}$ & $\begin{array}{l}0.006 \\
(0.012)\end{array}$ \\
\hline Manufacturing & & & $\begin{array}{c}-0.008 \\
(0.026)\end{array}$ & $\begin{array}{l}-0.012 \\
(0.034)\end{array}$ & $\begin{array}{c}-0.012 \\
(0.027)\end{array}$ & $\begin{array}{l}-0.016 \\
(0.034)\end{array}$ \\
\hline Construction & & & $\begin{array}{l}0.115^{\text {**** }} \\
(0.035)\end{array}$ & $\begin{array}{l}0.114^{* *} \\
(0.046)\end{array}$ & $\begin{array}{l}0.115^{* * *} \\
(0.035)\end{array}$ & $\begin{array}{l}0.114^{* *} \\
(0.046)\end{array}$ \\
\hline Wholesale and retail trade & & & $\begin{array}{l}0.054^{*} \\
(0.029)\end{array}$ & $\begin{array}{l}0.056 \\
(0.036)\end{array}$ & $\begin{array}{l}0.051^{*} \\
(0.029)\end{array}$ & $\begin{array}{l}0.052 \\
(0.036)\end{array}$ \\
\hline Hotel and restaurant & & & $\begin{array}{l}0.063^{* *} \\
(0.031)\end{array}$ & $\begin{array}{l}0.057 \\
(0.037)\end{array}$ & $\begin{array}{l}0.062^{* *} \\
(0.031)\end{array}$ & $\begin{array}{l}0.055 \\
(0.037)\end{array}$ \\
\hline Public and community services & & & $\begin{array}{l}0.154^{* * * *} \\
(0.026)\end{array}$ & $\begin{array}{l}0.161^{* * * *} \\
(0.034)\end{array}$ & $\begin{array}{l}0.162^{* * *} \\
(0.027)\end{array}$ & $\begin{array}{l}0.169^{* * *} \\
(0.034)\end{array}$ \\
\hline Education & & & $\begin{array}{l}0.132^{* * *} \\
(0.032)\end{array}$ & $\begin{array}{l}0.130^{* * * *} \\
(0.041)\end{array}$ & $\begin{array}{l}0.136^{* * *} \\
(0.032)\end{array}$ & $\begin{array}{l}0.135^{\text {*** }} \\
(0.041)\end{array}$ \\
\hline Health & & & $\begin{array}{l}0.120^{* * * *} \\
(0.029)\end{array}$ & $\begin{array}{l}0.120^{* * *} \\
(0.036)\end{array}$ & $\begin{array}{l}0.122^{* * * *} \\
(0.029)\end{array}$ & $\begin{array}{l}0.123^{* * *} \\
(0.036)\end{array}$ \\
\hline Urban area & & & $\begin{array}{l}-0.105^{* * *} \\
(0.018)\end{array}$ & $\begin{array}{l}-0.108^{* * *} \\
(0.023)\end{array}$ & $\begin{array}{l}-0.103^{* * *} \\
(0.018)\end{array}$ & $\begin{array}{l}-0.106^{* * *} \\
(0.023)\end{array}$ \\
\hline Unemployment to vacancy ratio & & & $\begin{array}{l}0.000 \\
(0.003)\end{array}$ & $\begin{array}{l}-0.001 \\
(0.004)\end{array}$ & $\begin{array}{l}0.001 \\
(0.003)\end{array}$ & $\begin{array}{l}-0.001 \\
(0.004)\end{array}$ \\
\hline Gender equality policy and practice & & & & & $\begin{array}{l}-0.015^{*} \\
(0.008)\end{array}$ & $\begin{array}{l}-0.017 \\
(0.011)\end{array}$ \\
\hline Constant & $\begin{array}{l}3.961^{* * *} \\
(0.057)\end{array}$ & $\begin{array}{l}3.956^{* * *} \\
(0.060)\end{array}$ & $\begin{array}{l}3.955^{* * *} \\
(0.070)\end{array}$ & $\begin{array}{l}3.955^{* * *} \\
(0.078)\end{array}$ & $\begin{array}{l}3.955^{* * *} \\
(0.070)\end{array}$ & $\begin{array}{l}3.954^{* * *} \\
(0.078)\end{array}$ \\
\hline No. of employees & 18,064 & 18,064 & 18,064 & 18,064 & 18,064 & 18,064 \\
\hline R-squared & 0.090 & & 0.095 & & 0.095 & \\
\hline No. of workplaces & & 1506 & & 1506 & & 1506 \\
\hline
\end{tabular}

Robust standard errors in parentheses.

${ }^{* * *} \mathrm{p}<0.01,{ }^{* *} \mathrm{p}<0.05$, and ${ }^{*} \mathrm{p}<0.1$. 
Table 6

Workplace gender diversity and affective well-being, sub-group analysis (female).

\begin{tabular}{|c|c|c|c|c|c|c|}
\hline & OLS & $\mathrm{RE}$ & OLS & $\mathrm{RE}$ & OLS & $\mathrm{RE}$ \\
\hline Gender diversity & $\begin{array}{l}-0.155^{* *} \\
(0.065)\end{array}$ & $\begin{array}{l}-0.148^{*} \\
(0.082)\end{array}$ & $\begin{array}{l}-0.122^{*} \\
(0.071)\end{array}$ & $\begin{array}{l}-0.116 \\
(0.087)\end{array}$ & $\begin{array}{l}-0.104 \\
(0.072)\end{array}$ & $\begin{array}{c}-0.097 \\
(0.088)\end{array}$ \\
\hline Majority female (female > 50\%) & $\begin{array}{l}-0.046^{* *} \\
(0.023)\end{array}$ & $\begin{array}{c}-0.038 \\
(0.028)\end{array}$ & $\begin{array}{l}-0.047^{*} \\
(0.025)\end{array}$ & $\begin{array}{l}-0.040 \\
(0.031)\end{array}$ & $\begin{array}{l}-0.048^{*} \\
(0.025)\end{array}$ & $\begin{array}{l}-0.041 \\
(0.031)\end{array}$ \\
\hline Age $<30$ & $\begin{array}{l}-0.054^{*} \\
(0.029)\end{array}$ & $\begin{array}{l}-0.046 \\
(0.029)\end{array}$ & $\begin{array}{l}-0.042 \\
(0.029)\end{array}$ & $\begin{array}{l}-0.039 \\
(0.029)\end{array}$ & $\begin{array}{l}-0.044 \\
(0.029)\end{array}$ & $\begin{array}{l}-0.040 \\
(0.029)\end{array}$ \\
\hline Age 30-39 & $\begin{array}{l}0.003 \\
(0.027)\end{array}$ & $\begin{array}{l}0.006 \\
(0.027)\end{array}$ & $\begin{array}{l}0.012 \\
(0.028)\end{array}$ & $\begin{array}{l}0.012 \\
(0.027)\end{array}$ & $\begin{array}{l}0.011 \\
(0.028)\end{array}$ & $\begin{array}{l}0.011 \\
(0.027)\end{array}$ \\
\hline Age $50+$ & $\begin{array}{l}0.119 * * * \\
(0.027)\end{array}$ & $\begin{array}{l}0.108^{* * *} \\
(0.027)\end{array}$ & $\begin{array}{l}0.109^{* * *} \\
(0.027)\end{array}$ & $\begin{array}{l}0.103^{* * *} \\
(0.027)\end{array}$ & $\begin{array}{l}0.108^{* * *} \\
(0.027)\end{array}$ & $\begin{array}{l}0.102^{* * *} \\
(0.027)\end{array}$ \\
\hline Married & $\begin{array}{c}-0.017 \\
(0.021)\end{array}$ & $\begin{array}{c}-0.018 \\
(0.021)\end{array}$ & $\begin{array}{l}-0.019 \\
(0.021)\end{array}$ & $\begin{array}{l}-0.020 \\
(0.021)\end{array}$ & $\begin{array}{l}-0.019 \\
(0.021)\end{array}$ & $\begin{array}{l}-0.020 \\
(0.021)\end{array}$ \\
\hline White & $\begin{array}{l}-0.087^{* *} \\
(0.043)\end{array}$ & $\begin{array}{l}-0.091^{* *} \\
(0.044)\end{array}$ & $\begin{array}{l}-0.100^{* *} \\
(0.044)\end{array}$ & $\begin{array}{l}-0.106^{* *} \\
(0.044)\end{array}$ & $\begin{array}{l}-0.102^{* *} \\
(0.044)\end{array}$ & $\begin{array}{l}-0.108^{* *} \\
(0.044)\end{array}$ \\
\hline Children $<7$ year old & $\begin{array}{l}0.011 \\
(0.029)\end{array}$ & $\begin{array}{l}0.012 \\
(0.029)\end{array}$ & $\begin{array}{l}0.006 \\
(0.029)\end{array}$ & $\begin{array}{l}0.009 \\
(0.029)\end{array}$ & $\begin{array}{l}0.008 \\
(0.029)\end{array}$ & $\begin{array}{l}0.010 \\
(0.029)\end{array}$ \\
\hline Other dependents & $\begin{array}{l}-0.108^{* * *} \\
(0.025)\end{array}$ & $\begin{array}{l}-0.109^{* * * *} \\
(0.025)\end{array}$ & $\begin{array}{l}-0.108^{* * *} \\
(0.025)\end{array}$ & $\begin{array}{l}-0.108^{* * * *} \\
(0.025)\end{array}$ & $\begin{array}{l}-0.107^{* * *} \\
(0.025)\end{array}$ & $\begin{array}{l}-0.108^{* * * *} \\
(0.025)\end{array}$ \\
\hline Disabled & $\begin{array}{l}-0.153^{* * *} \\
(0.031)\end{array}$ & $\begin{array}{l}-0.145^{* * *} \\
(0.030)\end{array}$ & $\begin{array}{l}-0.149^{* * * *} \\
(0.031)\end{array}$ & $\begin{array}{l}-0.142^{* * * *} \\
(0.030)\end{array}$ & $\begin{array}{l}-0.148^{* * *} \\
(0.031)\end{array}$ & $\begin{array}{l}-0.142^{* * * *} \\
(0.030)\end{array}$ \\
\hline No academic qualification & $\begin{array}{l}0.102^{* *} \\
(0.042)\end{array}$ & $\begin{array}{l}0.105^{* * *} \\
(0.041)\end{array}$ & $\begin{array}{l}0.122^{* * * *} \\
(0.042)\end{array}$ & $\begin{array}{l}0.119^{* * * *} \\
(0.041)\end{array}$ & $\begin{array}{l}0.121^{* * * *} \\
(0.042)\end{array}$ & $\begin{array}{l}0.118^{* * *} \\
(0.041)\end{array}$ \\
\hline O-level & $\begin{array}{l}0.089 * * * \\
(0.032)\end{array}$ & $\begin{array}{l}0.092^{* * *} \\
(0.032)\end{array}$ & $\begin{array}{l}0.106^{* * *} \\
(0.032)\end{array}$ & $\begin{array}{l}0.103^{\text {**** }} \\
(0.032)\end{array}$ & $\begin{array}{l}0.105^{* * *} \\
(0.032)\end{array}$ & $\begin{array}{l}0.102^{* * *} \\
(0.032)\end{array}$ \\
\hline A-level & $\begin{array}{l}0.033 \\
(0.039)\end{array}$ & $\begin{array}{l}0.033 \\
(0.039)\end{array}$ & $\begin{array}{l}0.041 \\
(0.039)\end{array}$ & $\begin{array}{l}0.039 \\
(0.039)\end{array}$ & $\begin{array}{l}0.041 \\
(0.039)\end{array}$ & $\begin{array}{l}0.038 \\
(0.039)\end{array}$ \\
\hline Other qualification & $\begin{array}{l}0.042 \\
(0.028)\end{array}$ & $\begin{array}{l}0.040 \\
(0.028)\end{array}$ & $\begin{array}{l}0.049^{*} \\
(0.028)\end{array}$ & $\begin{array}{l}0.044 \\
(0.028)\end{array}$ & $\begin{array}{l}0.048^{*} \\
(0.028)\end{array}$ & $\begin{array}{l}0.043 \\
(0.028)\end{array}$ \\
\hline On permanent contract & $\begin{array}{l}-0.062^{*} \\
(0.034)\end{array}$ & $\begin{array}{l}-0.050 \\
(0.034)\end{array}$ & $\begin{array}{l}-0.052 \\
(0.034)\end{array}$ & $\begin{array}{l}-0.043 \\
(0.035)\end{array}$ & $\begin{array}{l}-0.054 \\
(0.034)\end{array}$ & $\begin{array}{l}-0.044 \\
(0.035)\end{array}$ \\
\hline Full-time & $\begin{array}{l}-0.090^{* * * *} \\
(0.029)\end{array}$ & $\begin{array}{l}-0.094^{* * * *} \\
(0.028)\end{array}$ & $\begin{array}{l}-0.098^{* * *} \\
(0.029)\end{array}$ & $\begin{array}{l}-0.099^{* * *} \\
(0.028)\end{array}$ & $\begin{array}{l}-0.099^{* * *} \\
(0.029)\end{array}$ & $\begin{array}{l}-0.100^{\text {**** }} \\
(0.028)\end{array}$ \\
\hline Works over $48 \mathrm{~h}$ & $\begin{array}{l}-0.218^{* * *} \\
(0.023)\end{array}$ & $\begin{array}{l}-0.226^{* * * *} \\
(0.023)\end{array}$ & $\begin{array}{l}-0.232^{* * *} \\
(0.024)\end{array}$ & $\begin{array}{l}-0.234^{* * *} \\
(0.023)\end{array}$ & $\begin{array}{l}-0.232^{* * * *} \\
(0.024)\end{array}$ & $\begin{array}{l}-0.234^{* * *} \\
(0.023)\end{array}$ \\
\hline Skill same as required & $\begin{array}{l}0.074^{* * * *} \\
(0.019)\end{array}$ & $\begin{array}{l}0.068^{* * * *} \\
(0.019)\end{array}$ & $\begin{array}{l}0.075^{* * * *} \\
(0.019)\end{array}$ & $\begin{array}{l}0.069^{* * * *} \\
(0.019)\end{array}$ & $\begin{array}{l}0.075^{* * *} \\
(0.019)\end{array}$ & $\begin{array}{l}0.069^{* * *} \\
(0.019)\end{array}$ \\
\hline Professional & $\begin{array}{l}-0.035 \\
(0.043)\end{array}$ & $\begin{array}{l}-0.032 \\
(0.045)\end{array}$ & $\begin{array}{l}-0.034 \\
(0.045)\end{array}$ & $\begin{array}{l}-0.033 \\
(0.047)\end{array}$ & $\begin{array}{l}-0.034 \\
(0.045)\end{array}$ & $\begin{array}{l}-0.033 \\
(0.047)\end{array}$ \\
\hline Associate prof. and technical & $\begin{array}{l}0.041 \\
(0.040)\end{array}$ & $\begin{array}{l}0.041 \\
(0.041)\end{array}$ & $\begin{array}{l}0.030 \\
(0.040)\end{array}$ & $\begin{array}{l}0.035 \\
(0.041)\end{array}$ & $\begin{array}{l}0.030 \\
(0.040)\end{array}$ & $\begin{array}{l}0.035 \\
(0.041)\end{array}$ \\
\hline Admin. and secretarial & $\begin{array}{l}0.126^{* * *} \\
(0.040)\end{array}$ & $\begin{array}{l}0.141^{* * * *} \\
(0.040)\end{array}$ & $\begin{array}{l}0.129 * * * \\
(0.040)\end{array}$ & $\begin{array}{l}0.142^{* * * *} \\
(0.040)\end{array}$ & $\begin{array}{l}0.129 * * * \\
(0.040)\end{array}$ & $\begin{array}{l}0.143^{* * *} \\
(0.040)\end{array}$ \\
\hline Skilled trades plant and mach. & $\begin{array}{l}0.086 \\
(0.064)\end{array}$ & $\begin{array}{l}0.100 \\
(0.064)\end{array}$ & $\begin{array}{l}0.086 \\
(0.067)\end{array}$ & $\begin{array}{l}0.102 \\
(0.066)\end{array}$ & $\begin{array}{l}0.085 \\
(0.067)\end{array}$ & $\begin{array}{l}0.101 \\
(0.066)\end{array}$ \\
\hline Personal and customer services & $\begin{array}{l}0.127^{* * *} \\
(0.043)\end{array}$ & $\begin{array}{l}0.133^{* * *} \\
(0.043)\end{array}$ & $\begin{array}{l}0.118^{* * *} \\
(0.043)\end{array}$ & $\begin{array}{l}0.129 * * * \\
(0.043)\end{array}$ & $\begin{array}{l}0.119^{* * *} \\
(0.043)\end{array}$ & $\begin{array}{l}0.130^{* * *} \\
(0.043)\end{array}$ \\
\hline Elementary occupations & $\begin{array}{l}0.217^{* * *} \\
(0.052)\end{array}$ & $\begin{array}{l}0.225^{* * *} \\
(0.052)\end{array}$ & $\begin{array}{l}0.216^{* * *} \\
(0.053)\end{array}$ & $\begin{array}{l}0.226^{\text {**** }} \\
(0.052)\end{array}$ & $\begin{array}{l}0.215^{* * *} \\
(0.053)\end{array}$ & $\begin{array}{l}0.225^{* * *} \\
(0.052)\end{array}$ \\
\hline Gross weekly pay $\leq 110$ & $\begin{array}{l}0.321^{* * *} \\
(0.046)\end{array}$ & $\begin{array}{l}0.301^{* * *} \\
(0.047)\end{array}$ & $\begin{array}{l}0.293^{* * *} \\
(0.047)\end{array}$ & $\begin{array}{l}0.278^{* * * *} \\
(0.047)\end{array}$ & $\begin{array}{l}0.289^{* * *} \\
(0.047)\end{array}$ & $\begin{array}{l}0.274^{* * *} \\
(0.047)\end{array}$ \\
\hline Gross weekly pay $111-180$ & $\begin{array}{l}0.207^{* * *} \\
(0.040)\end{array}$ & $\begin{array}{l}0.188^{* * *} \\
(0.040)\end{array}$ & $\begin{array}{l}0.179^{\text {**** }} \\
(0.041)\end{array}$ & $\begin{array}{l}0.169^{* * * *} \\
(0.040)\end{array}$ & $\begin{array}{l}0.176^{* * *} \\
(0.041)\end{array}$ & $\begin{array}{l}0.166^{* * * *} \\
(0.040)\end{array}$ \\
\hline Gross weekly pay $181-260$ & $\begin{array}{l}0.139 * * * \\
(0.032)\end{array}$ & $\begin{array}{l}0.130^{* * * *} \\
(0.032)\end{array}$ & $\begin{array}{l}0.119^{* * * *} \\
(0.033)\end{array}$ & $\begin{array}{l}0.117^{\text {**** }} \\
(0.032)\end{array}$ & $\begin{array}{l}0.117^{* * *} \\
(0.033)\end{array}$ & $\begin{array}{l}0.115^{* * *} \\
(0.032)\end{array}$ \\
\hline Gross weekly pay 261-360 & $\begin{array}{l}0.033 \\
(0.029)\end{array}$ & $\begin{array}{l}0.024 \\
(0.029)\end{array}$ & $\begin{array}{l}0.019 \\
(0.029)\end{array}$ & $\begin{array}{l}0.015 \\
(0.029)\end{array}$ & $\begin{array}{l}0.018 \\
(0.029)\end{array}$ & $\begin{array}{l}0.014 \\
(0.029)\end{array}$ \\
\hline Trade union member & $\begin{array}{l}-0.168^{* * *} \\
(0.021)\end{array}$ & $\begin{array}{l}-0.151^{* * * *} \\
(0.023)\end{array}$ & $\begin{array}{l}-0.156^{* * *} \\
(0.023)\end{array}$ & $\begin{array}{l}-0.144^{* * *} \\
(0.024)\end{array}$ & $\begin{array}{l}-0.154^{* * *} \\
(0.023)\end{array}$ & $\begin{array}{l}-0.143^{\text {*** }} \\
(0.024)\end{array}$ \\
\hline Log workplace age & & & $\begin{array}{l}-0.016^{*} \\
(0.009)\end{array}$ & $\begin{array}{l}-0.020^{*} \\
(0.011)\end{array}$ & $\begin{array}{l}-0.016^{*} \\
(0.009)\end{array}$ & $\begin{array}{l}-0.020^{*} \\
(0.011)\end{array}$ \\
\hline Private establishment & & & $\begin{array}{l}0.115^{* * *} \\
(0.028)\end{array}$ & $\begin{array}{l}0.102^{* * *} \\
(0.035)\end{array}$ & $\begin{array}{l}0.110^{* * * *} \\
(0.029)\end{array}$ & $\begin{array}{l}0.095^{* * *} \\
(0.036)\end{array}$ \\
\hline Sole establishment & & & $\begin{array}{l}0.020 \\
(0.025)\end{array}$ & $\begin{array}{l}0.038 \\
(0.031)\end{array}$ & $\begin{array}{l}0.016 \\
(0.025)\end{array}$ & $\begin{array}{l}0.032 \\
(0.031)\end{array}$ \\
\hline No. of employees/1000 & & & $\begin{array}{l}-0.002 \\
(0.012)\end{array}$ & $\begin{array}{l}-0.005 \\
(0.015)\end{array}$ & $\begin{array}{l}0.003 \\
(0.012)\end{array}$ & $\begin{array}{l}0.002 \\
(0.016)\end{array}$ \\
\hline Manufacturing & & & $\begin{array}{l}0.044 \\
(0.045)\end{array}$ & $\begin{array}{l}0.033 \\
(0.053)\end{array}$ & $\begin{array}{l}0.038 \\
(0.046)\end{array}$ & $\begin{array}{l}0.026 \\
(0.053)\end{array}$ \\
\hline Construction & & & $\begin{array}{l}0.083 \\
(0.063)\end{array}$ & $\begin{array}{l}0.086 \\
(0.078)\end{array}$ & $\begin{array}{l}0.083 \\
(0.063)\end{array}$ & $\begin{array}{l}0.086 \\
(0.078)\end{array}$ \\
\hline Wholesale and retail trade & & & $\begin{array}{l}0.012 \\
(0.041)\end{array}$ & $\begin{array}{l}0.017 \\
(0.048)\end{array}$ & $\begin{array}{l}0.010 \\
(0.041)\end{array}$ & $\begin{array}{l}0.013 \\
(0.048)\end{array}$ \\
\hline Hotel and restaurant & & & $\begin{array}{l}0.044 \\
(0.047)\end{array}$ & $\begin{array}{l}0.040 \\
(0.054)\end{array}$ & $\begin{array}{l}0.041 \\
(0.048)\end{array}$ & $\begin{array}{l}0.036 \\
(0.054)\end{array}$ \\
\hline Public and community services & & & $\begin{array}{l}0.191^{* * *} \\
(0.037)\end{array}$ & $\begin{array}{l}0.190^{* * *} \\
(0.046)\end{array}$ & $\begin{array}{l}0.199 * * * \\
(0.037)\end{array}$ & $\begin{array}{l}0.200^{* * *} \\
(0.046)\end{array}$ \\
\hline
\end{tabular}


Table 6 (continued)

\begin{tabular}{|c|c|c|c|c|c|c|}
\hline & OLS & $\mathrm{RE}$ & OLS & $\mathrm{RE}$ & OLS & $\mathrm{RE}$ \\
\hline Education & & & $\begin{array}{l}0.173^{* * *} \\
(0.042)\end{array}$ & $\begin{array}{l}0.165^{* * * *} \\
(0.053)\end{array}$ & $\begin{array}{l}0.178^{* * * *} \\
(0.042)\end{array}$ & $\begin{array}{l}0.171^{* * *} \\
(0.053)\end{array}$ \\
\hline Health & & & $\begin{array}{l}0.160^{* * * *} \\
(0.037)\end{array}$ & $\begin{array}{l}0.148^{* * * *} \\
(0.045)\end{array}$ & $\begin{array}{l}0.165^{* * * *} \\
(0.037)\end{array}$ & $\begin{array}{l}0.153^{* * *} \\
(0.046)\end{array}$ \\
\hline Urban area & & & $\begin{array}{l}-0.116^{* * *} \\
(0.026)\end{array}$ & $\begin{array}{l}-0.122^{* * * *} \\
(0.033)\end{array}$ & $\begin{array}{l}-0.113^{* * *} \\
(0.026)\end{array}$ & $\begin{array}{l}-0.119^{* * *} \\
(0.033)\end{array}$ \\
\hline Unemployment to vacancy ratio & & & $\begin{array}{l}-0.003 \\
(0.004)\end{array}$ & $\begin{array}{l}-0.005 \\
(0.005)\end{array}$ & $\begin{array}{l}-0.003 \\
(0.004)\end{array}$ & $\begin{array}{l}-0.005 \\
(0.005)\end{array}$ \\
\hline Gender equality policy and practice & & & & & $\begin{array}{l}-0.017 \\
(0.011)\end{array}$ & $\begin{array}{l}-0.021 \\
(0.014)\end{array}$ \\
\hline Constant & $\begin{array}{l}3.944^{* * *} \\
(0.079)\end{array}$ & $\begin{array}{l}3.933^{* * *} \\
(0.084)\end{array}$ & $\begin{array}{l}3.929^{* * *} \\
(0.097)\end{array}$ & $\begin{array}{l}3.954^{* * * *} \\
(0.109)\end{array}$ & $\begin{array}{l}3.929^{* * * *} \\
(0.097)\end{array}$ & $\begin{array}{l}3.952^{* * *} \\
(0.109)\end{array}$ \\
\hline $\begin{array}{l}\text { No. of employees } \\
\text { R-squared }\end{array}$ & $\begin{array}{l}9450 \\
0.108\end{array}$ & 9450 & $\begin{array}{l}9450 \\
0.114\end{array}$ & 9450 & $\begin{array}{l}9450 \\
0.115\end{array}$ & 9450 \\
\hline No. of workplaces & & 1409 & & 1409 & & 1409 \\
\hline
\end{tabular}

Robust standard errors in parentheses.

${ }^{* * *} \mathrm{p}<0.01,{ }^{* *} \mathrm{p}<0.05$, and ${ }^{*} \mathrm{p}<0.1$.

Table 7

Workplace gender diversity and affective well-being, sub-group analysis (male).

\begin{tabular}{|c|c|c|c|c|c|c|}
\hline & OLS & $\mathrm{RE}$ & OLS & RE & OLS & $\mathrm{RE}$ \\
\hline Gender diversity & $\begin{array}{l}-0.031 \\
(0.080)\end{array}$ & $\begin{array}{l}-0.052 \\
(0.092)\end{array}$ & $\begin{array}{l}-0.009 \\
(0.083)\end{array}$ & $\begin{array}{l}-0.036 \\
(0.095)\end{array}$ & $\begin{array}{l}0.000 \\
(0.084)\end{array}$ & $\begin{array}{l}-0.025 \\
(0.095)\end{array}$ \\
\hline Majority female (female > 50\%) & $\begin{array}{l}-0.020 \\
(0.025)\end{array}$ & $\begin{array}{l}-0.023 \\
(0.028)\end{array}$ & $\begin{array}{c}-0.008 \\
(0.027)\end{array}$ & $\begin{array}{c}-0.012 \\
(0.032)\end{array}$ & $\begin{array}{c}-0.008 \\
(0.027)\end{array}$ & $\begin{array}{l}-0.012 \\
(0.032)\end{array}$ \\
\hline Age $<30$ & $\begin{array}{l}0.005 \\
(0.033)\end{array}$ & $\begin{array}{l}0.001 \\
(0.033)\end{array}$ & $\begin{array}{l}0.000 \\
(0.033)\end{array}$ & $\begin{array}{c}-0.002 \\
(0.033)\end{array}$ & $\begin{array}{l}-0.000 \\
(0.033)\end{array}$ & $\begin{array}{c}-0.002 \\
(0.033)\end{array}$ \\
\hline Age 30-39 & $\begin{array}{l}-0.012 \\
(0.028)\end{array}$ & $\begin{array}{l}-0.012 \\
(0.028)\end{array}$ & $\begin{array}{l}-0.012 \\
(0.028)\end{array}$ & $\begin{array}{l}-0.011 \\
(0.028)\end{array}$ & $\begin{array}{c}-0.012 \\
(0.028)\end{array}$ & $\begin{array}{l}-0.012 \\
(0.028)\end{array}$ \\
\hline Age $50+$ & $\begin{array}{l}0.160^{* * *} \\
(0.029)\end{array}$ & $\begin{array}{l}0.151^{* * *} \\
(0.028)\end{array}$ & $\begin{array}{l}0.158^{* * *} \\
(0.029)\end{array}$ & $\begin{array}{l}0.149 * * * \\
(0.028)\end{array}$ & $\begin{array}{l}0.158^{* * *} \\
(0.029)\end{array}$ & $\begin{array}{l}0.149^{* * *} \\
(0.028)\end{array}$ \\
\hline Married & $\begin{array}{l}0.007 \\
(0.025)\end{array}$ & $\begin{array}{l}0.003 \\
(0.024)\end{array}$ & $\begin{array}{l}0.006 \\
(0.025)\end{array}$ & $\begin{array}{l}0.003 \\
(0.024)\end{array}$ & $\begin{array}{l}0.007 \\
(0.025)\end{array}$ & $\begin{array}{l}0.003 \\
(0.024)\end{array}$ \\
\hline White & $\begin{array}{l}0.040 \\
(0.046)\end{array}$ & $\begin{array}{l}0.048 \\
(0.047)\end{array}$ & $\begin{array}{l}0.027 \\
(0.047)\end{array}$ & $\begin{array}{l}0.038 \\
(0.047)\end{array}$ & $\begin{array}{l}0.026 \\
(0.047)\end{array}$ & $\begin{array}{l}0.038 \\
(0.047)\end{array}$ \\
\hline Children $<7$ year old & $\begin{array}{l}-0.012 \\
(0.027)\end{array}$ & $\begin{array}{l}-0.011 \\
(0.027)\end{array}$ & $\begin{array}{l}-0.012 \\
(0.027)\end{array}$ & $\begin{array}{l}-0.012 \\
(0.027)\end{array}$ & $\begin{array}{l}-0.011 \\
(0.027)\end{array}$ & $\begin{array}{l}-0.011 \\
(0.027)\end{array}$ \\
\hline Other dependents & $\begin{array}{l}-0.101^{\text {**** }} \\
(0.030)\end{array}$ & $\begin{array}{l}-0.101^{\text {**** }} \\
(0.029)\end{array}$ & $\begin{array}{l}-0.101^{* * * *} \\
(0.030)\end{array}$ & $\begin{array}{l}-0.101^{* * * *} \\
(0.029)\end{array}$ & $\begin{array}{l}-0.101^{* * * *} \\
(0.030)\end{array}$ & $\begin{array}{l}-0.101^{* * *} \\
(0.029)\end{array}$ \\
\hline Disabled & $\begin{array}{l}-0.199^{* * *} \\
(0.031)\end{array}$ & $\begin{array}{l}-0.192^{* * *} \\
(0.030)\end{array}$ & $\begin{array}{l}-0.197^{* * * *} \\
(0.031)\end{array}$ & $\begin{array}{l}-0.191^{\text {**** }} \\
(0.030)\end{array}$ & $\begin{array}{l}-0.196^{* * *} \\
(0.031)\end{array}$ & $\begin{array}{l}-0.190^{* * *} \\
(0.030)\end{array}$ \\
\hline No academic qualification & $\begin{array}{l}0.160^{* * * *} \\
(0.039)\end{array}$ & $\begin{array}{l}0.152^{* * * *} \\
(0.038)\end{array}$ & $\begin{array}{l}0.155^{* * *} \\
(0.039)\end{array}$ & $\begin{array}{l}0.148^{* * *} \\
(0.038)\end{array}$ & $\begin{array}{l}0.153^{* * *} \\
(0.039)\end{array}$ & $\begin{array}{l}0.147^{* * *} \\
(0.038)\end{array}$ \\
\hline O-level & $\begin{array}{l}0.061^{*} \\
(0.032)\end{array}$ & $\begin{array}{l}0.056^{*} \\
(0.033)\end{array}$ & $\begin{array}{l}0.057^{*} \\
(0.033)\end{array}$ & $\begin{array}{l}0.054^{*} \\
(0.033)\end{array}$ & $\begin{array}{l}0.056^{*} \\
(0.033)\end{array}$ & $\begin{array}{l}0.053 \\
(0.033)\end{array}$ \\
\hline A-level & $\begin{array}{l}0.020 \\
(0.039)\end{array}$ & $\begin{array}{l}0.023 \\
(0.040)\end{array}$ & $\begin{array}{l}0.018 \\
(0.039)\end{array}$ & $\begin{array}{l}0.022 \\
(0.041)\end{array}$ & $\begin{array}{l}0.018 \\
(0.039)\end{array}$ & $\begin{array}{l}0.022 \\
(0.041)\end{array}$ \\
\hline Other qualification & $\begin{array}{l}0.020 \\
(0.027)\end{array}$ & $\begin{array}{l}0.019 \\
(0.028)\end{array}$ & $\begin{array}{l}0.020 \\
(0.028)\end{array}$ & $\begin{array}{l}0.020 \\
(0.029)\end{array}$ & $\begin{array}{l}0.020 \\
(0.028)\end{array}$ & $\begin{array}{l}0.020 \\
(0.029)\end{array}$ \\
\hline On permanent contract & $\begin{array}{l}-0.102^{* *} \\
(0.041)\end{array}$ & $\begin{array}{l}-0.087^{* *} \\
(0.042)\end{array}$ & $\begin{array}{l}-0.100^{* *} \\
(0.041)\end{array}$ & $\begin{array}{l}-0.085^{* *} \\
(0.042)\end{array}$ & $\begin{array}{l}-0.100^{* *} \\
(0.041)\end{array}$ & $\begin{array}{l}-0.085^{* *} \\
(0.042)\end{array}$ \\
\hline Full-time & $\begin{array}{l}-0.124^{* *} \\
(0.048)\end{array}$ & $\begin{array}{l}-0.123^{* * *} \\
(0.047)\end{array}$ & $\begin{array}{l}-0.115^{* *} \\
(0.048)\end{array}$ & $\begin{array}{l}-0.115^{* *} \\
(0.047)\end{array}$ & $\begin{array}{l}-0.115^{* *} \\
(0.048)\end{array}$ & $\begin{array}{l}-0.115^{* *} \\
(0.047)\end{array}$ \\
\hline Works over $48 \mathrm{~h}$ & $\begin{array}{l}-0.187^{* * * *} \\
(0.022)\end{array}$ & $\begin{array}{l}-0.191^{* * *} \\
(0.022)\end{array}$ & $\begin{array}{l}-0.195^{\text {**** }} \\
(0.023)\end{array}$ & $\begin{array}{l}-0.197^{* * * *} \\
(0.022)\end{array}$ & $\begin{array}{l}-0.195^{\text {**** }} \\
(0.023)\end{array}$ & $\begin{array}{l}-0.197^{\text {**** }} \\
(0.022)\end{array}$ \\
\hline Skill same as required & $\begin{array}{l}0.054^{* * *} \\
(0.020)\end{array}$ & $\begin{array}{l}0.050^{* *} \\
(0.020)\end{array}$ & $\begin{array}{l}0.049^{* *} \\
(0.020)\end{array}$ & $\begin{array}{l}0.047^{* *} \\
(0.020)\end{array}$ & $\begin{array}{l}0.049^{* *} \\
(0.020)\end{array}$ & $\begin{array}{l}0.047^{* *} \\
(0.020)\end{array}$ \\
\hline Professional & $\begin{array}{l}-0.024 \\
(0.035)\end{array}$ & $\begin{array}{l}-0.015 \\
(0.038)\end{array}$ & $\begin{array}{c}-0.013 \\
(0.036)\end{array}$ & $\begin{array}{l}-0.006 \\
(0.039)\end{array}$ & $\begin{array}{l}-0.014 \\
(0.036)\end{array}$ & $\begin{array}{l}-0.008 \\
(0.039)\end{array}$ \\
\hline Associate prof. and technical & $\begin{array}{l}0.087^{* *} \\
(0.034)\end{array}$ & $\begin{array}{l}0.079^{* *} \\
(0.036)\end{array}$ & $\begin{array}{l}0.082^{* *} \\
(0.034)\end{array}$ & $\begin{array}{l}0.075^{* *} \\
(0.036)\end{array}$ & $\begin{array}{l}0.082^{* *} \\
(0.034)\end{array}$ & $\begin{array}{l}0.076^{* *} \\
(0.036)\end{array}$ \\
\hline Admin. and secretarial & $\begin{array}{l}0.000 \\
(0.043)\end{array}$ & $\begin{array}{l}0.009 \\
(0.044)\end{array}$ & $\begin{array}{l}0.002 \\
(0.043)\end{array}$ & $\begin{array}{l}0.011 \\
(0.044)\end{array}$ & $\begin{array}{l}0.002 \\
(0.043)\end{array}$ & $\begin{array}{l}0.011 \\
(0.044)\end{array}$ \\
\hline Skilled trades plant and mach. & $\begin{array}{l}0.253^{* * *} \\
(0.035)\end{array}$ & $\begin{array}{l}0.260^{* * * *} \\
(0.036)\end{array}$ & $\begin{array}{l}0.251^{* * *} \\
(0.035)\end{array}$ & $\begin{array}{l}0.258^{* * *} \\
(0.036)\end{array}$ & $\begin{array}{l}0.250^{* * * *} \\
(0.035)\end{array}$ & $\begin{array}{l}0.257^{\text {**** }} \\
(0.036)\end{array}$ \\
\hline Personal and customer services & $\begin{array}{l}0.062 \\
(0.048)\end{array}$ & $\begin{array}{l}0.068 \\
(0.049)\end{array}$ & $\begin{array}{l}0.042 \\
(0.049)\end{array}$ & $\begin{array}{l}0.050 \\
(0.050)\end{array}$ & $\begin{array}{l}0.041 \\
(0.049)\end{array}$ & $\begin{array}{l}0.049 \\
(0.050)\end{array}$ \\
\hline Elementary occupations & $\begin{array}{l}0.221^{* * *} \\
(0.043)\end{array}$ & $\begin{array}{l}0.211^{* * *} \\
(0.043)\end{array}$ & $\begin{array}{l}0.223^{* * *} \\
(0.044)\end{array}$ & $\begin{array}{l}0.214^{* * *} \\
(0.043)\end{array}$ & $\begin{array}{l}0.222^{* * *} \\
(0.044)\end{array}$ & $\begin{array}{l}0.213^{* * *} \\
(0.043)\end{array}$ \\
\hline Gross weekly pay $\leq 110$ & $\begin{array}{l}0.357^{* * *} \\
(0.072)\end{array}$ & $\begin{array}{l}0.357^{* * *} \\
(0.074)\end{array}$ & $\begin{array}{l}0.330^{* * *} \\
(0.072)\end{array}$ & $\begin{array}{l}0.334^{* * *} \\
(0.075)\end{array}$ & $\begin{array}{l}0.327^{* * * *} \\
(0.072)\end{array}$ & $\begin{array}{l}0.331^{* * *} \\
(0.075)\end{array}$ \\
\hline
\end{tabular}


Table 7 (continued)

\begin{tabular}{|c|c|c|c|c|c|c|}
\hline & OLS & $\mathrm{RE}$ & OLS & $\mathrm{RE}$ & OLS & $\mathrm{RE}$ \\
\hline Gross weekly pay $111-180$ & $\begin{array}{l}0.156^{* *} \\
(0.064)\end{array}$ & $\begin{array}{l}0.167^{* * *} \\
(0.061)\end{array}$ & $\begin{array}{l}0.143^{* *} \\
(0.064)\end{array}$ & $\begin{array}{l}0.155^{* *} \\
(0.062)\end{array}$ & $\begin{array}{l}0.143^{* *} \\
(0.065)\end{array}$ & $\begin{array}{l}0.153^{* *} \\
(0.062)\end{array}$ \\
\hline Gross weekly pay $181-260$ & $\begin{array}{l}0.136^{* * *} \\
(0.038)\end{array}$ & $\begin{array}{l}0.148^{* * * *} \\
(0.036)\end{array}$ & $\begin{array}{l}0.133^{* * * *} \\
(0.038)\end{array}$ & $\begin{array}{l}0.145^{* * *} \\
(0.036)\end{array}$ & $\begin{array}{l}0.132^{* * *} \\
(0.038)\end{array}$ & $\begin{array}{l}0.144^{* * * *} \\
(0.036)\end{array}$ \\
\hline Gross weekly pay $261-360$ & $\begin{array}{l}0.023 \\
(0.028)\end{array}$ & $\begin{array}{l}0.031 \\
(0.028)\end{array}$ & $\begin{array}{l}0.026 \\
(0.028)\end{array}$ & $\begin{array}{l}0.033 \\
(0.028)\end{array}$ & $\begin{array}{l}0.025 \\
(0.028)\end{array}$ & $\begin{array}{l}0.032 \\
(0.028)\end{array}$ \\
\hline Trade union member & $\begin{array}{l}-0.116^{* * * *} \\
(0.022)\end{array}$ & $\begin{array}{l}-0.116^{* * * *} \\
(0.023)\end{array}$ & $\begin{array}{l}-0.102^{* * * *} \\
(0.025)\end{array}$ & $\begin{array}{l}-0.105^{\text {**** }} \\
(0.025)\end{array}$ & $\begin{array}{l}-0.100^{* * * *} \\
(0.025)\end{array}$ & $\begin{array}{l}-0.103^{* * * *} \\
(0.025)\end{array}$ \\
\hline Log workplace age & & & $\begin{array}{l}-0.014 \\
(0.009)\end{array}$ & $\begin{array}{l}-0.014 \\
(0.011)\end{array}$ & $\begin{array}{l}-0.014 \\
(0.009)\end{array}$ & $\begin{array}{l}-0.014 \\
(0.011)\end{array}$ \\
\hline Private establishment & & & $\begin{array}{l}0.043 \\
(0.033)\end{array}$ & $\begin{array}{l}0.040 \\
(0.037)\end{array}$ & $\begin{array}{l}0.037 \\
(0.033)\end{array}$ & $\begin{array}{l}0.034 \\
(0.038)\end{array}$ \\
\hline Sole establishment & & & $\begin{array}{l}0.042^{*} \\
(0.025)\end{array}$ & $\begin{array}{l}0.045 \\
(0.029)\end{array}$ & $\begin{array}{l}0.038 \\
(0.025)\end{array}$ & $\begin{array}{l}0.040 \\
(0.030)\end{array}$ \\
\hline No. of employees/1000 & & & $\begin{array}{l}0.006 \\
(0.014)\end{array}$ & $\begin{array}{l}0.004 \\
(0.016)\end{array}$ & $\begin{array}{l}0.013 \\
(0.015)\end{array}$ & $\begin{array}{l}0.012 \\
(0.018)\end{array}$ \\
\hline Manufacturing & & & $\begin{array}{l}-0.010 \\
(0.034)\end{array}$ & $\begin{array}{l}-0.005 \\
(0.041)\end{array}$ & $\begin{array}{l}-0.013 \\
(0.035)\end{array}$ & $\begin{array}{l}-0.009 \\
(0.041)\end{array}$ \\
\hline Construction & & & $\begin{array}{l}0.127^{* * * *} \\
(0.043)\end{array}$ & $\begin{array}{l}0.124^{* *} \\
(0.054)\end{array}$ & $\begin{array}{l}0.126^{* * * *} \\
(0.043)\end{array}$ & $\begin{array}{l}0.124^{* *} \\
(0.053)\end{array}$ \\
\hline Wholesale and retail trade & & & $\begin{array}{l}0.120^{* * *} \\
(0.042)\end{array}$ & $\begin{array}{l}0.118^{* *} \\
(0.049)\end{array}$ & $\begin{array}{l}0.117^{\text {*** }} \\
(0.042)\end{array}$ & $\begin{array}{l}0.114^{* *} \\
(0.049)\end{array}$ \\
\hline Hotel and restaurant & & & $\begin{array}{l}0.071^{*} \\
(0.041)\end{array}$ & $\begin{array}{l}0.069 \\
(0.047)\end{array}$ & $\begin{array}{l}0.070^{*} \\
(0.041)\end{array}$ & $\begin{array}{l}0.068 \\
(0.047)\end{array}$ \\
\hline Public and community services & & & $\begin{array}{l}0.109 * * * \\
(0.038)\end{array}$ & $\begin{array}{l}0.112^{* *} \\
(0.045)\end{array}$ & $\begin{array}{l}0.116^{* * *} \\
(0.039)\end{array}$ & $\begin{array}{l}0.120^{* * *} \\
(0.045)\end{array}$ \\
\hline Education & & & $\begin{array}{l}0.062 \\
(0.054)\end{array}$ & $\begin{array}{l}0.065 \\
(0.061)\end{array}$ & $\begin{array}{l}0.065 \\
(0.054)\end{array}$ & $\begin{array}{l}0.069 \\
(0.061)\end{array}$ \\
\hline Health & & & $\begin{array}{l}0.028 \\
(0.054)\end{array}$ & $\begin{array}{l}0.046 \\
(0.059)\end{array}$ & $\begin{array}{l}0.029 \\
(0.054)\end{array}$ & $\begin{array}{l}0.047 \\
(0.059)\end{array}$ \\
\hline Urban area & & & $\begin{array}{l}-0.099^{* * *} \\
(0.025)\end{array}$ & $\begin{array}{l}-0.099^{* * *} \\
(0.030)\end{array}$ & $\begin{array}{l}-0.098^{* * *} \\
(0.025)\end{array}$ & $\begin{array}{l}-0.097^{* * *} \\
(0.030)\end{array}$ \\
\hline Unemployment to vacancy ratio & & & $\begin{array}{l}0.005 \\
(0.004)\end{array}$ & $\begin{array}{l}0.005 \\
(0.005)\end{array}$ & $\begin{array}{l}0.005 \\
(0.004)\end{array}$ & $\begin{array}{l}0.005 \\
(0.005)\end{array}$ \\
\hline Gender equality policy and practice & & & & & $\begin{array}{l}-0.015 \\
(0.012)\end{array}$ & $\begin{array}{l}-0.016 \\
(0.015)\end{array}$ \\
\hline Constant & $\begin{array}{l}3.876 * * * \\
(0.087)\end{array}$ & $\begin{array}{l}3.864^{* * *} \\
(0.090)\end{array}$ & $\begin{array}{l}3.889^{* * * *} \\
(0.106)\end{array}$ & $\begin{array}{l}3.873^{* * *} \\
(0.112)\end{array}$ & $\begin{array}{l}3.888^{* * *} \\
(0.106)\end{array}$ & $\begin{array}{l}3.872^{* * *} \\
(0.112)\end{array}$ \\
\hline No. of employees & $\begin{array}{l}8614 \\
0074\end{array}$ & 8614 & 8614 & 8614 & 8614 & 8614 \\
\hline $\begin{array}{l}\text { R-squared } \\
\text { No. of workplaces }\end{array}$ & 0.074 & 1336 & 0.079 & 1336 & 0.080 & 1336 \\
\hline
\end{tabular}

Robust standard errors in parentheses.

${ }^{* * *} \mathrm{p}<0.01,{ }^{* *} \mathrm{p}<0.05$, and ${ }^{*} \mathrm{p}<0.1$

Table 8

Workplace gender diversity and affective well-being, group size related effect.

\begin{tabular}{|c|c|c|c|c|c|c|}
\hline & OLS & $\mathrm{RE}$ & OLS & RE & OLS & RE \\
\hline \multirow[t]{2}{*}{ Gender diversity } & -0.099 & -0.114 & -0.069 & -0.092 & -0.063 & -0.084 \\
\hline & $(0.076)$ & $(0.092)$ & $(0.079)$ & $(0.095)$ & $(0.079)$ & $(0.095)$ \\
\hline \multirow[t]{2}{*}{ Majority female (female > 50\%) } & -0.020 & -0.016 & -0.009 & -0.012 & -0.013 & -0.017 \\
\hline & $(0.042)$ & $(0.049)$ & $(0.048)$ & $(0.056)$ & $(0.048)$ & $(0.056)$ \\
\hline \multirow[t]{2}{*}{ Gender diversity*Majority female } & -0.040 & -0.056 & -0.071 & -0.069 & -0.059 & -0.058 \\
\hline & $(0.102)$ & $(0.125)$ & $(0.110)$ & $(0.133)$ & $(0.110)$ & $(0.133)$ \\
\hline \multirow[t]{2}{*}{ Age $<30$} & -0.029 & -0.028 & -0.026 & -0.027 & -0.027 & -0.027 \\
\hline & $(0.022)$ & $(0.021)$ & $(0.022)$ & $(0.022)$ & $(0.022)$ & $(0.022)$ \\
\hline \multirow[t]{2}{*}{ Age 30-39 } & -0.005 & -0.004 & -0.001 & -0.001 & -0.001 & -0.002 \\
\hline & $(0.020)$ & $(0.019)$ & $(0.020)$ & $(0.019)$ & $(0.020)$ & $(0.019)$ \\
\hline \multirow[t]{2}{*}{ Age $50+$} & $0.140^{* * *}$ & $0.127^{* * *}$ & $0.134^{* * *}$ & $0.124^{* * *}$ & $0.134^{* * * *}$ & $0.124^{* * * *}$ \\
\hline & $(0.020)$ & $(0.019)$ & $(0.020)$ & $(0.019)$ & $(0.020)$ & $(0.019)$ \\
\hline \multirow[t]{2}{*}{ Female } & $-0.087^{* * *}$ & $-0.081^{* * *}$ & $-0.087^{* * *}$ & $-0.082^{* * *}$ & $-0.087^{* * * *}$ & $-0.082^{* * *}$ \\
\hline & $(0.018)$ & $(0.017)$ & $(0.018)$ & $(0.018)$ & $(0.018)$ & $(0.017)$ \\
\hline \multirow[t]{2}{*}{ Married } & -0.005 & -0.008 & -0.005 & -0.008 & -0.005 & -0.008 \\
\hline & $(0.016)$ & $(0.016)$ & $(0.016)$ & $(0.016)$ & $(0.016)$ & $(0.016)$ \\
\hline \multirow[t]{2}{*}{ White } & -0.022 & -0.026 & -0.036 & -0.038 & -0.037 & -0.039 \\
\hline & $(0.032)$ & $(0.032)$ & $(0.032)$ & $(0.032)$ & $(0.032)$ & $(0.032)$ \\
\hline \multirow[t]{2}{*}{ Children $<7$ year old } & -0.004 & -0.002 & -0.005 & -0.002 & -0.004 & -0.002 \\
\hline & $(0.020)$ & $(0.020)$ & $(0.020)$ & $(0.020)$ & $(0.020)$ & $(0.020)$ \\
\hline \multirow[t]{2}{*}{ Other dependents } & $-0.105^{* * *}$ & $-0.106^{* * *}$ & $-0.105^{* * *}$ & $-0.106^{* * *}$ & $-0.104^{* * *}$ & $-0.105^{* * *}$ \\
\hline & $(0.019)$ & $(0.019)$ & $(0.019)$ & $(0.019)$ & $(0.019)$ & $(0.019)$ \\
\hline \multirow[t]{2}{*}{ Disabled } & $-0.178^{* * *}$ & $-0.170^{* * *}$ & $-0.174^{* * *}$ & $-0.169^{* * *}$ & $-0.174^{* * * *}$ & $-0.168^{* * * *}$ \\
\hline & $(0.022)$ & $(0.021)$ & $(0.022)$ & $(0.021)$ & $(0.022)$ & $(0.021)$ \\
\hline
\end{tabular}


Table 8 (continued)

\begin{tabular}{|c|c|c|c|c|c|c|}
\hline & OLS & $\mathrm{RE}$ & OLS & RE & OLS & $\mathrm{RE}$ \\
\hline No academic qualification & $\begin{array}{l}0.134^{* * *} \\
(0.028)\end{array}$ & $\begin{array}{l}0.133^{* * *} \\
(0.028)\end{array}$ & $\begin{array}{l}0.143^{* * *} \\
(0.029)\end{array}$ & $\begin{array}{l}0.138^{* * * *} \\
(0.028)\end{array}$ & $\begin{array}{l}0.141^{\text {*** }} \\
(0.029)\end{array}$ & $\begin{array}{l}0.137^{* * *} \\
(0.028)\end{array}$ \\
\hline O-level & $\begin{array}{l}0.082^{* * *} \\
(0.023)\end{array}$ & $\begin{array}{l}0.082^{* * *} \\
(0.023)\end{array}$ & $\begin{array}{l}0.089^{* * *} \\
(0.023)\end{array}$ & $\begin{array}{l}0.085^{\text {**** }} \\
(0.023)\end{array}$ & $\begin{array}{l}0.088^{* * *} \\
(0.023)\end{array}$ & $\begin{array}{l}0.085^{\text {*** }} \\
(0.023)\end{array}$ \\
\hline A-level & $\begin{array}{l}0.027 \\
(0.027)\end{array}$ & $\begin{array}{l}0.031 \\
(0.028)\end{array}$ & $\begin{array}{l}0.031 \\
(0.027)\end{array}$ & $\begin{array}{l}0.034 \\
(0.028)\end{array}$ & $\begin{array}{l}0.031 \\
(0.027)\end{array}$ & $\begin{array}{l}0.034 \\
(0.028)\end{array}$ \\
\hline Other qualification & $\begin{array}{l}0.036^{*} \\
(0.019)\end{array}$ & $\begin{array}{l}0.035^{*} \\
(0.020)\end{array}$ & $\begin{array}{l}0.039^{* *} \\
(0.020)\end{array}$ & $\begin{array}{l}0.037^{*} \\
(0.020)\end{array}$ & $\begin{array}{l}0.039^{* *} \\
(0.020)\end{array}$ & $\begin{array}{l}0.037^{*} \\
(0.020)\end{array}$ \\
\hline On permanent contract & $\begin{array}{l}-0.080^{* * *} \\
(0.026)\end{array}$ & $\begin{array}{l}-0.066^{* *} \\
(0.026)\end{array}$ & $\begin{array}{l}-0.072^{* * *} \\
(0.026)\end{array}$ & $\begin{array}{l}-0.061^{* *} \\
(0.027)\end{array}$ & $\begin{array}{l}-0.073^{* * *} \\
(0.026)\end{array}$ & $\begin{array}{l}-0.061^{* *} \\
(0.027)\end{array}$ \\
\hline Full-time & $\begin{array}{l}-0.109^{* * *} \\
(0.024)\end{array}$ & $\begin{array}{l}-0.105^{\text {**** }} \\
(0.024)\end{array}$ & $\begin{array}{l}-0.110^{\text {**** }} \\
(0.024)\end{array}$ & $\begin{array}{l}-0.105^{\text {**** }} \\
(0.024)\end{array}$ & $\begin{array}{l}-0.110^{\text {**** }} \\
(0.024)\end{array}$ & $\begin{array}{l}-0.106^{* * *} \\
(0.024)\end{array}$ \\
\hline Works over $48 \mathrm{~h}$ & $\begin{array}{l}-0.204^{* * *} \\
(0.016)\end{array}$ & $\begin{array}{l}-0.208^{* * *} \\
(0.016)\end{array}$ & $\begin{array}{l}-0.213^{* * *} \\
(0.016)\end{array}$ & $\begin{array}{l}-0.214^{* * *} \\
(0.016)\end{array}$ & $\begin{array}{l}-0.214^{* * *} \\
(0.016)\end{array}$ & $\begin{array}{l}-0.215^{* * *} \\
(0.016)\end{array}$ \\
\hline Skill same as required & $\begin{array}{l}0.063^{* * *} \\
(0.014)\end{array}$ & $\begin{array}{l}0.059^{* * * *} \\
(0.014)\end{array}$ & $\begin{array}{l}0.062^{* * *} \\
(0.014)\end{array}$ & $\begin{array}{l}0.058^{* * *} \\
(0.014)\end{array}$ & $\begin{array}{l}0.062^{* * * *} \\
(0.014)\end{array}$ & $\begin{array}{l}0.058^{* * *} \\
(0.014)\end{array}$ \\
\hline Professional & $\begin{array}{l}-0.031 \\
(0.027)\end{array}$ & $\begin{array}{l}-0.024 \\
(0.029)\end{array}$ & $\begin{array}{l}-0.029 \\
(0.028)\end{array}$ & $\begin{array}{l}-0.023 \\
(0.030)\end{array}$ & $\begin{array}{l}-0.030 \\
(0.028)\end{array}$ & $\begin{array}{l}-0.023 \\
(0.030)\end{array}$ \\
\hline Associate prof. and technical & $\begin{array}{l}0.064^{* *} \\
(0.025)\end{array}$ & $\begin{array}{l}0.054^{* *} \\
(0.027)\end{array}$ & $\begin{array}{l}0.054^{* *} \\
(0.026)\end{array}$ & $\begin{array}{l}0.049 * \\
(0.027)\end{array}$ & $\begin{array}{l}0.055^{* *} \\
(0.026)\end{array}$ & $\begin{array}{l}0.049 * \\
(0.027)\end{array}$ \\
\hline Admin. and secretarial & $\begin{array}{l}0.103^{* * * *} \\
(0.027)\end{array}$ & $\begin{array}{l}0.106^{* * * *} \\
(0.028)\end{array}$ & $\begin{array}{l}0.103^{* * *} \\
(0.027)\end{array}$ & $\begin{array}{l}0.107^{* * * *} \\
(0.028)\end{array}$ & $\begin{array}{l}0.104^{* * * *} \\
(0.027)\end{array}$ & $\begin{array}{l}0.107^{* * * *} \\
(0.028)\end{array}$ \\
\hline Skilled trades plant and mach. & $\begin{array}{l}0.226^{* * *} \\
(0.030)\end{array}$ & $\begin{array}{l}0.234^{* * *} \\
(0.030)\end{array}$ & $\begin{array}{l}0.225^{* * *} \\
(0.030)\end{array}$ & $\begin{array}{l}0.233^{* * *} \\
(0.030)\end{array}$ & $\begin{array}{l}0.224^{* * *} \\
(0.030)\end{array}$ & $\begin{array}{l}0.233^{* * *} \\
(0.030)\end{array}$ \\
\hline Personal and customer services & $\begin{array}{l}0.113^{* * * *} \\
(0.030)\end{array}$ & $\begin{array}{l}0.115^{\text {**** }} \\
(0.031)\end{array}$ & $\begin{array}{l}0.097^{* * * *} \\
(0.030)\end{array}$ & $\begin{array}{l}0.106^{* * *} \\
(0.031)\end{array}$ & $\begin{array}{l}0.097^{* * *} \\
(0.030)\end{array}$ & $\begin{array}{l}0.106^{* * *} \\
(0.031)\end{array}$ \\
\hline Elementary occupations & $\begin{array}{l}0.215^{* * *} \\
(0.033)\end{array}$ & $\begin{array}{l}0.208^{* * * *} \\
(0.033)\end{array}$ & $\begin{array}{l}0.219^{* * * *} \\
(0.033)\end{array}$ & $\begin{array}{l}0.213^{* * *} \\
(0.033)\end{array}$ & $\begin{array}{l}0.218^{* * *} \\
(0.033)\end{array}$ & $\begin{array}{l}0.212^{* * *} \\
(0.033)\end{array}$ \\
\hline Gross weekly pay $\leq 110$ & $\begin{array}{l}0.321^{* * *} \\
(0.037)\end{array}$ & $\begin{array}{l}0.318^{* * *} \\
(0.038)\end{array}$ & $\begin{array}{l}0.296^{* * *} \\
(0.038)\end{array}$ & $\begin{array}{l}0.298^{* * *} \\
(0.038)\end{array}$ & $\begin{array}{l}0.293^{* * *} \\
(0.038)\end{array}$ & $\begin{array}{l}0.296^{* * *} \\
(0.038)\end{array}$ \\
\hline Gross weekly pay $111-180$ & $\begin{array}{l}0.193^{* * *} \\
(0.033)\end{array}$ & $\begin{array}{l}0.190^{\text {**** }} \\
(0.032)\end{array}$ & $\begin{array}{l}0.176^{* * *} \\
(0.033)\end{array}$ & $\begin{array}{l}0.176^{* * *} \\
(0.032)\end{array}$ & $\begin{array}{l}0.173^{* * *} \\
(0.033)\end{array}$ & $\begin{array}{l}0.174^{* * *} \\
(0.032)\end{array}$ \\
\hline Gross weekly pay $181-260$ & $\begin{array}{l}0.135^{* * *} \\
(0.024)\end{array}$ & $\begin{array}{l}0.143^{* * *} \\
(0.024)\end{array}$ & $\begin{array}{l}0.126^{* * * *} \\
(0.024)\end{array}$ & $\begin{array}{l}0.136^{* * * *} \\
(0.024)\end{array}$ & $\begin{array}{l}0.124^{* * * *} \\
(0.024)\end{array}$ & $\begin{array}{l}0.134^{* * *} \\
(0.024)\end{array}$ \\
\hline Gross weekly pay $261-360$ & $\begin{array}{l}0.030 \\
(0.020)\end{array}$ & $\begin{array}{l}0.035^{*} \\
(0.020)\end{array}$ & $\begin{array}{l}0.028 \\
(0.020)\end{array}$ & $\begin{array}{l}0.033 \\
(0.020)\end{array}$ & $\begin{array}{l}0.027 \\
(0.020)\end{array}$ & $\begin{array}{l}0.032 \\
(0.020)\end{array}$ \\
\hline Trade union member & $\begin{array}{l}-0.144^{* * *} \\
(0.015)\end{array}$ & $\begin{array}{l}-0.138^{* * *} \\
(0.016)\end{array}$ & $\begin{array}{l}-0.135^{* * *} \\
(0.017)\end{array}$ & $\begin{array}{l}-0.131^{* * * *} \\
(0.017)\end{array}$ & $\begin{array}{l}-0.133^{* * * *} \\
(0.017)\end{array}$ & $\begin{array}{l}-0.129^{\text {**** }} \\
(0.017)\end{array}$ \\
\hline Log workplace age & & & $\begin{array}{l}-0.013^{* *} \\
(0.006)\end{array}$ & $\begin{array}{l}-0.012 \\
(0.008)\end{array}$ & $\begin{array}{l}-0.013^{* *} \\
(0.006)\end{array}$ & $\begin{array}{l}-0.012 \\
(0.008)\end{array}$ \\
\hline Private establishment & & & $\begin{array}{l}0.086^{* * *} \\
(0.021)\end{array}$ & $\begin{array}{l}0.085^{* * *} \\
(0.026)\end{array}$ & $\begin{array}{l}0.081^{* * *} \\
(0.022)\end{array}$ & $\begin{array}{l}0.079^{* * *} \\
(0.027)\end{array}$ \\
\hline Sole establishment & & & $\begin{array}{l}0.034^{*} \\
(0.018)\end{array}$ & $\begin{array}{l}0.042 * \\
(0.022)\end{array}$ & $\begin{array}{l}0.030^{*} \\
(0.018)\end{array}$ & $\begin{array}{l}0.037^{*} \\
(0.022)\end{array}$ \\
\hline No. of employees/1000 & & & $\begin{array}{l}-0.000 \\
(0.009)\end{array}$ & $\begin{array}{l}0.000 \\
(0.012)\end{array}$ & $\begin{array}{l}0.005 \\
(0.009)\end{array}$ & $\begin{array}{l}0.007 \\
(0.012)\end{array}$ \\
\hline Manufacturing & & & $\begin{array}{l}-0.006 \\
(0.027)\end{array}$ & $\begin{array}{l}-0.009 \\
(0.034)\end{array}$ & $\begin{array}{l}-0.010 \\
(0.027)\end{array}$ & $\begin{array}{l}-0.014 \\
(0.034)\end{array}$ \\
\hline Construction & & & $\begin{array}{l}0.120^{* * * *} \\
(0.036)\end{array}$ & $\begin{array}{l}0.118^{* *} \\
(0.047)\end{array}$ & $\begin{array}{l}0.118^{* * *} \\
(0.036)\end{array}$ & $\begin{array}{l}0.117^{* *} \\
(0.047)\end{array}$ \\
\hline Wholesale and retail trade & & & $\begin{array}{l}0.055^{*} \\
(0.029)\end{array}$ & $\begin{array}{l}0.056 \\
(0.036)\end{array}$ & $\begin{array}{l}0.052^{*} \\
(0.029)\end{array}$ & $\begin{array}{l}0.053 \\
(0.036)\end{array}$ \\
\hline Hotel and restaurant & & & $\begin{array}{l}0.066^{* *} \\
(0.031)\end{array}$ & $\begin{array}{l}0.059 \\
(0.037)\end{array}$ & $\begin{array}{l}0.064^{* *} \\
(0.031)\end{array}$ & $\begin{array}{l}0.057 \\
(0.037)\end{array}$ \\
\hline Public and community services & & & $\begin{array}{l}0.155^{* * * *} \\
(0.026)\end{array}$ & $\begin{array}{l}0.161^{* * * *} \\
(0.034)\end{array}$ & $\begin{array}{l}0.162^{* * *} \\
(0.027)\end{array}$ & $\begin{array}{l}0.169 * * * \\
(0.034)\end{array}$ \\
\hline Education & & & $\begin{array}{l}0.130^{* * * *} \\
(0.032)\end{array}$ & $\begin{array}{l}0.128^{* * *} \\
(0.041)\end{array}$ & $\begin{array}{l}0.134^{* * *} \\
(0.032)\end{array}$ & $\begin{array}{l}0.133^{* * *} \\
(0.041)\end{array}$ \\
\hline Health & & & $\begin{array}{l}0.116^{* * *} \\
(0.030)\end{array}$ & $\begin{array}{l}0.117^{* * *} \\
(0.037)\end{array}$ & $\begin{array}{l}0.119^{* * *} \\
(0.030)\end{array}$ & $\begin{array}{l}0.120^{* * *} \\
(0.037)\end{array}$ \\
\hline Urban area & & & $\begin{array}{l}-0.105^{* * * *} \\
(0.018)\end{array}$ & $\begin{array}{l}-0.109^{* * *} \\
(0.023)\end{array}$ & $\begin{array}{l}-0.104^{* * *} \\
(0.018)\end{array}$ & $\begin{array}{l}-0.107^{\text {*** }} \\
(0.023)\end{array}$ \\
\hline Unemployment to vacancy ratio & & & $\begin{array}{l}0.000 \\
(0.003)\end{array}$ & $\begin{array}{l}-0.001 \\
(0.004)\end{array}$ & $\begin{array}{l}0.001 \\
(0.003)\end{array}$ & $\begin{array}{l}-0.001 \\
(0.004)\end{array}$ \\
\hline Gender equality policy and practice & & & & & $\begin{array}{l}-0.015^{*} \\
(0.008)\end{array}$ & $\begin{array}{l}-0.017 \\
(0.011)\end{array}$ \\
\hline Constant & $\begin{array}{l}3.954^{* * *} \\
(0.060)\end{array}$ & $\begin{array}{l}3.946 * * * \\
(0.064)\end{array}$ & $\begin{array}{l}3.941^{* * * *} \\
(0.073)\end{array}$ & $\begin{array}{l}3.941^{* * * *} \\
(0.082)\end{array}$ & $\begin{array}{l}3.944^{* * * *} \\
(0.073)\end{array}$ & $\begin{array}{l}3.942 * * * \\
(0.082)\end{array}$ \\
\hline $\begin{array}{l}\text { No. of employees } \\
\text { R-squared }\end{array}$ & $\begin{array}{l}18,064 \\
0.090\end{array}$ & 18,064 & $\begin{array}{l}18,064 \\
0.095\end{array}$ & 18,064 & $\begin{array}{l}18,064 \\
0.095\end{array}$ & 18,064 \\
\hline No. of workplaces & & 1506 & & 1506 & & 1506 \\
\hline
\end{tabular}

Robust standard errors in parentheses.

${ }^{* * * *} \mathrm{p}<0.01,{ }^{* *} \mathrm{p}<0.05$, and ${ }^{*} \mathrm{p}<0.1$. 


\section{References}

Abowd, J., Kramarz, F., Margolis, D., 1999. High wage workers and high wage firms. Econometrica 67 (2), 251-333.

Aigner, D., Cain, G., 1977. Statistical theories of discrimination in labour markets. Industrial and Labour Relations Review 30 (2), 175-187.

Akerlof, G.A., Kranton, R.E., 2000. Economics and identity. Quarterly Journal of Economics 115 (3), 715-753.

Akrelof, G.A., Rose, A.K., Yellen, J.L., 1988. Job switching and job satisfaction in the US labour market. Brooking Papers on Economic Activity 2, 495-582.

Alesina, A., La Ferrara, E., 2000. Participation in heterogeneous communities. Quarterly Journal of Economics 115 (3), 847-904.

Alesina, A., La Ferrara, E., 2005. Ethnic diversity and economic performance. Journal of Economic Literature 43, 762-800.

Arrow, K., 1972. Models of job discrimination. In: Pascal, A. (Ed.), Racial Discrimination in Economic Life. D. C. Heath and Co, Lexington, Massachusetts.

Arrow, K., 1973. The theory of discrimination. In: Ashenfelter, Rees (Eds.), Discrimination in Labor Markets. Princeton University Press, Princeton.

Arulampalam, W., Booth, A., Bryan, M.L., 2007. Is there a glass ceiling over Europe? Exploring the gender pay gap across the wages distribution. Industrial \& Labor Relations Review 60 (2), 163-186.

Becker, G., 1957. The Economics of Discrimination. University of Chicago Press.

Berthoud, R., Blekesaune, M., 2007. Persistent employment disadvantage. Department for Work and Pensions Research Report No. 416.

Blalock, H., 1967. Toward a Theory of Minority-Groups. John Wiley \& Sons Inc.

Blau, F., DeVaro, J., 2007. New evidence on gender differences in promotion rates: an empirical analysis of a sample of new hires. Industrial Relations 46 (3), 511-550.

Booth, A., 2009. Gender and competition. Labour Economics 16, 599-606.

Bryson, A., Dale-Olsen, H., Barth, E., 2009. How does innovation affect worker wellbeing? CEP Discussion Paper No 953. LSE.

Clark, A., 2001. What really matters in a job? Hedonic measurement using quit data. Labour Economics 8, 223-242.

Clark, A., Georgellis, Y., Sanfey, P., 1998. Job satisfaction, wage changes, and quits. Research in Labour Economics 17, 95-121.

Daniels, K., 2000. Measures of five aspects of affective well-being at work. Human Relations 53 (2), 275-294.

Fields, D.L., Blum, T.C., 1997. Employee satisfaction in work groups with different gender composition. Journal of Organizational Behavior 18, 181-196.

Freeman, R., 1978. Job satisfaction as an economic variable. American Economic Review 68 (2), 135-141.

Freeman, R., Medoff, J., 1984. What Do Unions Do? New York: Basic Books.

Giuliano, L., Leonard, J., Levine, D., 2006. Do race, gender and age differences affect manageremployee relations? An analysis of quits, dismissals and promotions at a large retail firm, Institute for Research on Labor and Employment. Hiring in the English Labor Market. BE Press Advances in Economic Analysis \& Policy 6 (2) Article 1.

Jackson, S.E., Joshi, A., Erhardt, N.L., 2003. Recent research on team and organizational diversity: SWOT analysis and implications. Journal of Management 29 (6), 801-830.

Jones, M.K., Latreille, P.L., Sloane, P.J., 2003. Disability, gender and the labour market. IZA Discussion Paper No. 936.

Kersley, B., Alpin, C., Forth, J., Bryson, A., Bewley, H., Dix, G., Oxenbridge, S., 2006. Inside the Workplace: Findings from the 2004 Workplace Employment Relations Survey. Routledge, London.
Knippenberg, D., Schippers, M., 2007. Work group diversity. Annual Review of Psychology $58,515-541$.

Kochan, T., Bezrukova, K., Ely, R., Jackson, S., Joshi, A., Jehn, K., Leonard, J., Levine, D., Thomas, D., 2003. The effects of diversity on business performance: report of the diversity research work. Human Resource Management 42 (1), 3-21.

Kurtulus, F., 2008. The Effect of Heterogeneity on the Performance of Employees and Organizational Divisions of the Firm. Department of Economics, University of Massachusetts Amherst.

Lang, K., 1986. A language theory of discrimination. Quarterly Journal of Economics 101 (2), 363-382 May 1986.

Lazear, E., 1999. Globalisation and the market for team-mates. The Economic Journal 109, C15-C40.

Leonard, J.S., Levine, D.I., 2006. The effect of diversity on turnover: a large case study Industrial \& Labor Relations Review 59 (4), 547-572.

Levy-Garboua, L., Montmarquette, C., 2004. Reported job satisfaction: what does it mean? Journal of Socio-Economics 33 (2), 135-151.

Levy-Garboua, L., Montmarquette, C., Simonnet, V., 2007. Job satisfaction and quits: theory and evidence from the German socioeconomic panel. Labour Economics 14 (2), 251-268.

Maume, D.J., Sebastian, R., 2007. Racial composition of workgroups and job satisfaction among whites. The ANNALS of the American Academy of Political and Social Sciences 609, 85-103.

Office for National Statistics, 2006. Projections of the UK Labour Force 2006-2020.

Peccei, R., Lee, H., 2005. The impact of gender similarity on employee satisfaction at work: a review and re-evaluation. Journal of Management Studies 42 (8), 1571-1592.

Phelps, E.S., 1972. The statistical theory of racism and sexism. American Economic Review 62, 659-661.

Pudney, S., Shields, M., 2000a. Gender, race, pay and promotion in the British nursing profession: estimation of a generalised ordered probit model. Journal of Applied Econometrics 15, 367-399.

Pudney, S., Shields, M., 2000b. Gender and racial differentials in pay and promotion for NHS nurses. Oxford Bulletin of Economics and Statistics 62, 01-833.

Riach, P., Rich, J., 2006. An Experimental Investigation of Sexual Discrimination in Hiring in the English Labor Market. The B.E. Journal of Economic Analysis and Policy 6 (2), Article 1.

Rode, J., 2004. Job satisfaction and life satisfaction revisited: a longitudinal test of an integrated model. Human Relations 57 (9), 1205-1230.

Rose, M., 2005. Job satisfaction in Britain: coping with complexity. British Journal of Industrial Relations 43 (3), 55-467.

Warr, P., 1990. The measurement of well-being and other aspects of mental health Journal of Occupational Psychology 63 (3), 193-210.

Warr, P., 1994. Conceptual framework for the study of work and mental health. Work and Stress 8, 84-97.

Warr, P., 1999. Well-being and the workplace. In: Kahneman, D., Diener, E., Schwarz, N (Eds.), Well-being: The Foundations of Hedonic Psychology. Russell Sage Foundation, New York.

Wharton, A., Baron, J., 1987. So happy together? The impact of gender segregation on men at work. American Sociological Review 52, 574-587.

Wood, S., 2008. Job characteristics, employee voice and well-being in Britain. Industrial Relations Journal 39 (2), 153-168.

Wright, R.E., Ermisch, J.F., 1991. Gender discrimination in the British labour market: a reassessment. The Economic Journal 101, 508-522. 